\title{
Entropy and isoperimetry for linear and non-linear group actions
}

\author{
Misha Gromov
}

\begin{abstract}
We prove inequalities of isoperimetric type for groups acting on linear spaces and discuss related geometric and combinatorial problems, where we use the Boltzmann entropy to keep track of the cardinalities (and/or measures) of sets and of the dimensions of linear spaces.
\end{abstract}

Mathematics Subject Classification (2000). 20B07, 51Fxx, 52A37.

Keywords. Isoperimetric inequalities, amenable groups, group algebras, entropy.

\section{Contents}

1 Concepts and notation . . . . . . . . . . . . . . . . . . . . . . . 499

2 Non-amenability of groups and strengthened non-amenabilityof group algebras . . 518

3 Isoperimetry in the group algebras of ordered groups . . . . . . . . . . . . . . . . 524

4 Families of measurable partitions and their invariants . . . . . . . . . . . . 529

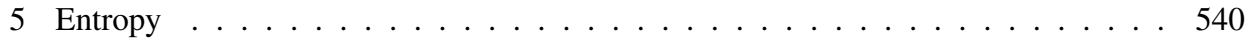

6 Lower bounds on $F_{*}$ for groups with large orderable subgroups . . . . . . . . . . 563

7 Linear algebraic entropic inequalities for normal group extensions . . . . . . . . . 569

8 Examples of group algebras with fast and withslow growing Følner functions . . . 574

$9 \quad E_{\circ}$-functions and isoperimetric inequalities for wreath powers of partitions and groups . . . . . . . . . . . . . . . . . . . . . 578

10 Brunn-Minkowski inequalities . . . . . . . . . . . . . . . 585

References . . . . . . . . . . . . . . . . . . . 592

\section{Concepts and notation}

1.1. Combinatorial isoperimetric profiles $I_{\circ}, I_{\circ}^{\text {incr }}$ and $I_{\circ}^{\text {max }}$. Consider a measure space $X$ (e.g. a discrete set with all points carrying unit weights) where the measure of a subset $Y \subset X$ is denoted by $|Y|$ (that is the cardinality for the discrete spaces with unitary atoms). 
If $g: X \rightarrow X$ is an invertible measure preserving map we define the $g$-boundary of $Y \in X$ as the set difference

$$
\partial_{g}(Y)=g(Y) \backslash Y .
$$

If $G$ is a set of such maps $g: X \rightarrow X$, define the ( joint exterior) $G$-boundary $\partial_{G}(Y)=\partial_{G}^{\text {joint }}(Y) \subset X$ of $Y \subset X$ as the union

$$
\partial_{G}(Y)=\bigcup_{g \in G} \partial_{g}(Y)=(G \cdot Y) \backslash Y
$$

and observe that

$$
\left|\partial_{G}(Y)\right|=|(G \cdot Y)|-|Y|,
$$

provided that $G \ni$ id.

An isoperimetric inequality in this context is the inequality of the form

$$
\left|\partial_{G}(Y)\right| \geq I(|Y|)
$$

where (o) is supposed to hold for all subsets $Y \subset X$ of finite measure (just finite subsets in the discrete case) with a given function $I=I(r)$ of real argument $r \geq 0$.

The combinatorial isoperimetric profile of $X$ with respect to $G$ is the maximal function, denoted

$$
I_{\circ}(r)=I_{\circ}(r ; X, G)
$$

for which all $Y$ satisfy (o).

Combinatorial isoperimetric problem: Evaluate $I_{\circ}$ and find extremal subsets $Y \subset$ $X$ that minimize $|\partial(Y)|$ among all $Y$ of given cardinality/measure. (The classical solution to this problem in the Euclidean plane is given by the round discs $Y$ bounded by circles $\partial(Y)$.)

$\Gamma$-spaces $X$. This means that $X$ is a Borel measure space with a group $\Gamma$ acting from the left on $X$ by measure preserving transformations. For instance, $X$ may be equal to $\Gamma$ with the left action, where $\Gamma$ is a countable (or locally compact) group with a given finite (compact) subset $G \subset \Gamma$ that is usually (but not always) assumed to generate $\Gamma$.

$I_{\circ}(r)$ for real numbers $r$. It is convenient to have functions of positive integer arguments extended to all $r \in \mathbb{R}_{+}$; we tacitly assume such extension with the functions $I_{\circ}(r)$ (and similar functions we shall meet later on) being constant or linear on the integer intervals $(i, i+1)$.

Asymptotic equivalence. If $\Gamma$ is an infinite finitely generated group then the combinatorial isoperimetric profiles for different finite generating subsets $G, G^{\prime} \subset \Gamma$ are asymptotically equivalent, i.e., their ratio is bounded away from 0 and $\infty$.

In fact if $G \subset G^{\prime} \subset G^{\cdot m}$, where $G^{\cdot m} \subset \Gamma$ denotes the set of the words in the letters $g \in G$ of length $\leq m$, then the corresponding profiles satisfy $I \leq I^{\prime} \leq m \cdot\left|G^{\prime}\right| \cdot I$. 
This equivalence class is then regarded as the combinatorial isoperimetric profile of $\Gamma$ that does not depend on $G$.

Sublinearity of $I_{\circ}$. The function $I_{\circ}(r)=I_{\circ}(r ; \Gamma, G)$ is bounded by $|G| r$ and it satisfies the inequality

$$
I_{\circ}\left(r_{1}+r_{2}\right) \leq I_{\circ}\left(r_{1}\right)+I_{\circ}\left(r_{2}\right)
$$

for all infinite groups $\Gamma$ and finite subsets $G \subset \Gamma$.

Non-monotonicity of $I_{\circ}$ and $I_{\circ}^{\text {incr }}$. The profile $I_{\circ}(r)$ of a group $\Gamma$ is not always monotone increasing in $r$. For example, $I_{\circ}(r \geq|\Gamma|)=0$ for finite groups $\Gamma$. If we insist on the monotonicity, we define

$$
I_{\circ}^{\text {incr }}(r)=\inf _{s \geq r} I_{\circ}(s)
$$

that is the maximal monotone increasing minorant of $I_{\circ}$.

Possibly, the ratio $I_{\circ}(r) / I_{\circ}^{\text {incr }}(r)$ is bounded for all infinite finitely generated groups.

Remark. The notion of the isoperimetric profile for groups was introduced by Anatoly Vershik [29] in the (essentially equivalent) language of the Følner functions defined below.

1.2. $I_{\circ}$ for subgroups, for factor groups and for measures on groups. The inequality $(+)_{\circ}$ implies that the $G$-isoperimetric profile of a free action of an infinite group $(\Gamma, G)$ on an arbitrary space (set) $X$ equals the $G$-profile of $\Gamma$, since the boundary of a subset in $X$ equals the disjoint union of the boundaries of the orbits. Consequently,

the profile of every group extension $\left(\Gamma_{1} \supset \Gamma, G_{1} \supset G\right)$ is bounded from below by the profile of $(\Gamma, G)$,

$$
I_{\circ}\left(r ; \Gamma_{1}, G_{1}\right) \geq I_{\circ}(r ; \Gamma, G) .
$$

Also

$I_{\circ}$ decreases under epimorphisms of infinite groups.

Namely, let $h: \Gamma \rightarrow \Gamma$ be a surjective group homomorphism, $G$ a finite subset in $\Gamma$ and let $\underline{G}=h(G) \subset \underline{\Gamma}$ be the image of $G$ in $\underline{\Gamma}$. Then

$$
I_{\circ}(r ; \Gamma, G) \geq I_{\circ}(r ; \underline{\Gamma}, \underline{G}) .
$$

Proof. Given $Y \subset \Gamma$, let $\underline{Y}_{m} \subset \underline{\Gamma}, m=1,2, \ldots$, be the subset of points with at least $m$ preimages in $Y$. Clearly,

$$
\left|\partial_{G}(Y)\right| \geq \sum_{m=1,2, \ldots}\left|\partial_{\underline{G}}\left(\underline{Y}_{m}\right)\right|
$$


and the subadditivity applies, since

$$
\sum_{m=1,2, \ldots}\left|\underline{Y}_{m}\right|=|Y| .
$$

Subgraphs of functions and their boundaries. Given a positive function $f(x)$ on a space $X$, define the subgraph $Y^{f} \subset X \times \mathbb{R}_{+}$as the set of pairs $(x, r)$, such that $f(x) \leq r$. Then, for a set $G$ of transformations of $X$, let

$$
\left|\partial_{G}(f)\right|:=\left|\partial_{G}\left(Y^{f}\right)\right|
$$

for the obvious action of $G$ on $X \times \mathbb{R}_{+}$.

If the function $f$ is integer-valued, then $\left|\partial_{G}(f)\right|$ equals the sum of the $G$-boundaries of its levels $Y_{m}:=\{f(x)=m\} \subset X, m=0,1,2, \ldots$,

$$
\left|\partial_{G}(f)\right|=\sum_{m}\left|\partial_{G}\left(Y_{m}\right)\right| ;
$$

thus one obtains the isoperimetric lower bound on $\left|\partial_{G}(f)\right|$ by the $l_{1}$-norm $\|f\|_{l_{1}}:=$ $\left|Y^{f}\right|$ of $f$,

$$
\left|\partial_{G}(f)\right| \geq I_{\circ}\left(\|f\|_{l_{1}} ; G\right),
$$

provided that $I_{\circ}$ is subadditive. (A similar inequality for non-integer-valued functions needs extra entropic data on $f$, see 5.12.)

This applies especially to the function $\underline{f}(\underline{\gamma})=\left|h^{-1}(\underline{\gamma})\right|$ for the above $h: \Gamma \rightarrow \underline{\Gamma}$, where the essential feature is the (obvious) inequality $\left|\partial_{\underline{G}}(f)\right| \leq\left|\partial_{G}(Y)\right|$.

Generalization to maps between $\Gamma$-spaces. Let $X$ and $\underline{X}$ be spaces acted upon by $\Gamma$ and let $X \rightarrow \underline{X}$ be a $\Gamma$-equivariant map, where a basic example is $X=\Gamma$ and $\underline{X}=\Gamma / \Gamma_{0}$. The function $I_{\circ}(r ; \underline{X})$ is not always subadditive, e.g. for some quotients $\Gamma / \Gamma_{0}$ where $\Gamma$ is the free group $F_{2}$. If this is the case we work with the maximal subadditive minorant $I_{\circ}^{\text {sb.ad }}(r)$ of $I_{\circ}(r)$ and observe with the above argument that

$$
I_{\circ}^{\text {sb.ad }}(r ; X) \geq I_{\circ}^{\text {sb.ad }}(r ; \underline{X}) .
$$

1.3. Boundaries $|\partial|^{\max }, \partial_{\mu}$ and the associated isoperimetric profiles. The boundary of a $Y \subset X$ can be regarded as a function of $g \in G$ for $g \mapsto \partial_{g}(Y)$ and, besides taking the union of these $g$-boundaries, one may take the supremum of their cardinalities and, thus, define $|\partial|^{\max }(Y)$ with $I_{\circ}^{\max }(r, \Gamma, G)$ denoting the corresponding profile.

Another useful boundary is the $\mu$-boundary $\left|\partial_{\mu}(Y)\right|=\sum_{g} \mu(g)\left|\partial_{g}(Y)\right|$ for a measure $\mu$ on $G$, with $I_{\circ}(r ; \Gamma, \mu)$ for the associated profile.

If $\mu$ equals the Haar measure of $\Gamma$ restricted to a subset $H \subset \Gamma$ (this $H$ may be different from the original $G$ when it comes to applications ), this boundary is denoted 
$\left|\partial_{\Sigma H}\right|(Y)$. If the group is discrete with the Haar measure having unitary atoms, then

$$
\left|\partial_{\Sigma H}\right|(Y)=|Y| \cdot|H|-\sum_{g \in H}|Y \cap g(Y)|=|Y| \cdot|H|-\sum_{Y \times Y}\left|y_{1} y_{2}^{-1}\right|_{H},
$$

where $\left|y_{1} y_{2}^{-1}\right|_{H}$ denotes the number of $g \in H$ such that $g\left(y_{1}\right)=y_{2}$.

All these boundaries lead to the equivalent isoperimetric profiles for individual $G$, but they behave differently for families $G_{i}$, where $\left|G_{i}\right| \rightarrow \infty$.

The essential properties of these boundaries are quite similar. For example, all of them except for $|\partial|^{\max }$ are slice-wise additive for actions of $\Gamma$ on $X$ with several orbits (slices) $S$ :

$|\partial|$ of every $Y \subset X$ equals the sum $\sum_{S}|\partial|(Y \cap S)$.

In what follows, everything applies to all types of boundaries unless stated otherwise.

The boundary $|\partial|^{\max }$, albeit non-additive, is more convenient than $\partial^{\text {joint }}$ for Cartesian products of groups (and spaces) as is seen in the following

$\mathbb{Z}^{2}$-Example. Consider the group $\Gamma=\mathbb{Z}^{2}=\mathbb{Z} \times \mathbb{Z}$ with the standard generators $g_{1}$ and $g_{2}$. Then every $Y \subset \mathbb{Z}^{2}$ obviously satisfies $|Y| \leq|\partial|^{\max }(Y)^{2}$, where the extremal sets are the $n \times n$-squares. But if we turn to the joint exterior boundary, we see that the diamond, the square rotated by $90^{\circ}$, has this boundary $\sqrt{2}$-times smaller than the square, while the cardinalities of the sets in $\mathbb{Z}^{2}$ do not change under the rotations away from the boundary.

1.4. $G^{\cdot m}$-boundary and Coulhon-Saloff-Coste inequality. Start with the following triangle inequality:

$$
\left|\partial_{g_{1} \cdot g_{2}}(Y)\right| \leq\left|\partial_{g_{1}}(Y)\right|+\left|\partial_{g_{2}}(Y)\right|,
$$

for every pair of invertible transformations $g_{1}$ and $g_{2}$ of $X$.

To see this clearer, look at the " $X$-cube" $Z=2^{X}$, i.e., the set of all finite subsets $Y \subset X$ with $|Y|<\infty$ where the distance is given by the maximum of the measures (or the cardinalities) of the differences of subsets (representing non-symmetric metric on the set of subsets) and is denoted

$$
\left|Y_{1}-Y_{2}\right|:=\max \left(\left|Y_{1} \backslash Y_{2}\right|,\left|Y_{2} \backslash Y_{1}\right|\right) .
$$

Since transformations $g$ of $X$ isometrically act on $Z$ and $\partial_{g}(Y)=|Y-g(Y)|$, the inequality $\left(\partial_{g_{1}} \cdot g_{2}\right)$ is a consequence of the triangle inequality in $2^{X}$.

It follows that

the boundary of every subset $Y \subset X$ with respect to the $m$-ball $G^{\cdot m} \subset \Gamma$ satisfies

$$
\left.\frac{1}{m}|\partial|_{G^{m}}^{\max }(Y)|\leq| \partial\right|_{G} ^{\max }(Y) .
$$


On the other hand, if the action of $\Gamma$ on $X$ is free, for $H=G^{\cdot m}$ the above identity $\Sigma G$ implies that

$$
|\partial|_{G^{m}}^{\max }\left|(Y) \geq\left(1-\frac{|Y|}{\left|G^{\cdot m}\right|}\right)\right| Y \mid
$$

and yields the following inequality due to Coulhon-Saloff-Coste [4].

\section{CSC displacement inequality.}

$$
|\partial|_{G}^{\max }(Y) \geq \frac{1}{m}\left(1-\frac{|Y|}{\left|G^{\cdot m}\right|}\right)|Y|
$$

for all $Y \subset \Gamma$ and all $m=1,2, \ldots$

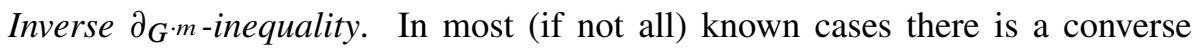
inequality saying that the function $I(r, m)=I_{\circ}^{\max }\left(r ; G^{\cdot m}\right)$ is, roughly, linear in $m$, i.e., $I(r, m) \sim m \cdot I(r, 1)$ for $m \leq r / 2 I(r, 1)$.

It is unclear if this is true in general and what are more general constraints on possible ( $\Gamma$-invariant) metrics on the orbits $\Gamma(Y) \subset 2^{\Gamma}$ for various $Y \subset \Gamma$.

On the other hand, (DIS) admits the following (strengthened) generalization.

Intersection inequality. A subset $H \subset \Gamma$ is called (right) $G$-connected if every two points $h_{0}$ and $h$ in $H$ can be joined by a chain of points $\left(h_{0}, h_{1}, \ldots, h_{i}, \ldots, h_{k}=h\right)$ such that $h_{i}=h_{i-1} g_{i}$ for some $g_{i} \in G$ and all $i=1,2, \ldots, k$. Observe that the subsets $H=G^{\cdot m} \subset \Gamma$ are $G$-connected for all $m=1,2, \ldots$; moreover, if $G^{-1}=G$ and $G \ni$ id, then every two points in $G^{\cdot m}$ are contained in a $G$-connected subset of cardinality $\leq 2 m$.

If a finite subset $H \subset \Gamma$ is $G$-connected for a given $G \subset \Gamma$, then all measurable subsets $Y$ of an arbitrary $\Gamma$-space $X$ (with the action written "on the left": $x \mapsto \gamma x)$ satisfy

$$
\left|\bigcap_{h \in H} h(Y)\right| \geq|Y|-(|H|-1)|\partial|_{G}^{\max }(Y) .
$$

Proof. Observe that $\left|\bigcap_{h \in H} h(Y)\right|=|Y|$ for $|H|=1$ and proceed by induction on $|H|$ for $|H|>1$. To do this, decompose $H=H^{\prime} \cup\left\{h_{0} g\right\}$ for a $G$-connected subset $H^{\prime} \subset H$ with $\left|H^{\prime}\right|=|H|-1$ and some $h_{0} \in H^{\prime}$ and $g \in G$, and notice that the intersection inequality is stable under the left translations of $H$.

Thus, we pass from $H$ to $h_{0}^{-1} H$ and arrive at the case where $h_{0}=\mathrm{id} \in H^{\prime}$ and $H=H^{\prime} \cup\{g\}$, and where, obviously,

$$
\left|\left(\bigcap_{h \in H^{\prime}} h(Y)\right) \bigcap g(Y)\right| \geq \bigcap_{h \in H^{\prime}} h(Y)|-|(Y \backslash g(Y))|=| \bigcap_{h \in H^{\prime}} h(Y)|-| \partial_{g}(Y) \mid .
$$


1.5. Linear algebraic isoperimetric profiles $I_{*}$ of groups and algebras. Let $L$ be a linear space over some field $\mathbb{F}$ and $G$ a set of linear operators $L \rightarrow L$, where in our examples these operators are invertible, injective as well as surjective. We define the (joint exterior) $G$-boundary of a linear subspace $D \subset L$ as the quotient space

$$
\partial_{G}(D)=\partial_{G}^{\text {joint }}(D)=(G * D) / D,
$$

where $G * D \subset L$ denotes the linear span of the subspaces $g(D)$ for all $g \in G$ and where $A / B:=A / A \cap B$. Set

$$
\left|\partial_{G}(D)\right|=\operatorname{rank}_{\mathbb{F}} \partial_{G}(D)
$$

(that equals $\operatorname{rank}_{\mathbb{F}}(G * D)-\operatorname{rank}_{\mathbb{F}}(D)$ if $G \ni$ id) and define the isoperimetric profile of $L$ with respect to $G$ as the maximal function $I_{*}$ such that all finite dimensional subspaces $D \subset L$ satisfy the following "isoperimetric inequality",

$$
\left|\partial_{G}(D)\right| \geq I_{*}(|D|),
$$

where $|D|$ stands for $\operatorname{rank}(D)=\operatorname{rank}_{\mathbb{F}}(D)$.

Main examples: group algebras and their extensions. Let $L=A$ be the group algebra of $\Gamma$ over some field $\mathbb{F}$ regarded as the vector space of the $\mathbb{F}$-valued functions on $\Gamma$ with finite supports. The group $\Gamma$ linearly acts on $A$ and, for a given subset $G \subset \Gamma$, one may speak of the $G$-boundary $\partial_{G}$ in $A$ as well as in $\Gamma$.

Supports of linear spaces and the implication $(*) \Longrightarrow(0)$. The support of a linear space $D$ of functions on $\Gamma$ is, by definition, the complement to the set where all functions from $D$ vanish. If $|\operatorname{supp}(D)|=|D|$ then $D$ equals the space $\mathbb{F}^{Y}$ of all functions on $Y=\operatorname{supp}(D)$ and, obviously, $\left|\partial_{G}(D)\right|=\left|\partial_{G}(Y)\right|$. Thus,

$$
I_{\circ} \geq I_{*} .
$$

The reverse inequality does not always hold:

There are finitely generated groups (with torsion), where the two profiles are not equivalent; for example $I_{*}(r)$ can be bounded while $r / I_{\circ}(r)$ grows arbitrarily (depending on $\Gamma$ ) slowly (see 8.1).

It is possible, albeit unlikely, that $I_{*}(r) \sim I_{\circ}(r)$ for groups without torsion. (This is similar to the Kaplansky conjecture saying that the group algebras of torsion-free groups have no divisors of zero.)

Sublinearity and monotonicity of $I_{*}$ of group algebras. Clearly,

$$
I_{*}\left(r_{1}+r_{2}\right) \leq I_{*}\left(r_{1}\right)+I_{*}\left(r_{2}\right)
$$

for infinite groups $\Gamma$; therefore, 
the profile of a group algebra of an infinite group may only increase under a group extension.

Also we shall prove in 3.5 that

$I_{*}$ decreases under surjective group homomorphisms when the target group is left orderable.

It remains unclear to what extend this continues to be valid in general, but we shall see in 7.2 that some entropic isoperimetric profile is always monotone decreasing under surjective group homomorphisms of infinite groups.

Remark. The profile $I_{*}$ is residually monotone: if $\Gamma_{i} \subset \Gamma$ is a decreasing sequence of normal subgroups such that $\bigcap_{i} \Gamma_{i}=\{\mathrm{id}\}$, then $I_{*}(r ; \Gamma) \geq \lim _{i \rightarrow \infty} I_{*}\left(r ; \Gamma / \Gamma_{i}\right)$, since the push-forward homomorphism of the group algebra $A$ of $\Gamma$ to the algebra $A_{i}$ of $\Gamma / \Gamma_{i}$ is injective on each finite dimensional subspace $D \subset A$ for large $i=i(D)$. (This remains true for the algebra of $l_{1}$-functions on $\Gamma$.)

About dependence of $I_{*}$ on $\mathbb{F}$. The action of $G \ni$ id acting on $r$ functions $X \rightarrow \mathbb{F}$ is described by the following infinite system of linear equations in unknowns $f_{j x y}=f_{j}(\gamma(x))$, indexed by $(x \in X, \gamma \in G, j=1,2, \ldots, r)$,

$$
f_{j \gamma(x) \mathrm{id}}=f_{j x \gamma} .
$$

The bound $I_{*}(r) \leq a$ means that there is a linear space $D^{+}$of solutions of LIN in functions with finite supports such that $\left|D^{+}\right| \leq r+a$, while the map from $D^{+}$to the space of $r$-tuples of functions on $x$ given by $f_{j x y} \mapsto f_{j}(x)$ has rank $r$.

Thus, by the Lefschetz principle,

if $\mathbb{F}$ is algebraically closed, then the profile $I_{*}$ for $\mathbb{F}$-functions with finite supports on any $\Gamma$-space $X$ depends only on the characteristics of $\mathbb{F}$.

( $I_{*}$ may depend on $\mathbb{F}$, e.g. for finite groups, but the extent of this dependence is unclear.)

1.6. The boundaries $|\partial|^{\max },|\partial|_{\mu}$ and $|\partial|_{G} * m$. One defines $|\partial|^{\max }$ and $|\partial|_{\mu}$ as in the combinatorial case with the corresponding notation $I_{*}^{\max }(r ; \Gamma, G)$ and $I_{*}(r ; \Gamma, \mu)$ for the isoperimetric profiles and one observes that

$$
\left.\frac{1}{m}|\partial|_{G^{m}}^{\max }(D)|\leq| \partial\right|_{G} ^{\max }(D) . \quad\left(\partial_{G^{* m}}\right)
$$

Thus,

$$
I_{*}^{\max }(r ; G) \geq \frac{1}{m} I_{*}^{\max }\left(r ; G^{\cdot m}\right),
$$


where the triangle inequality applies to the metric on the set (Grassmannian) of subspaces $D$ in $L$ defined by

$$
\left|D_{1}-D_{2}\right|:=\frac{1}{2}\left(\left|D_{1} / D_{2}\right|+\left|D_{2} / D_{1}\right|\right) .
$$

Furthermore,

$$
\left|\bigcap_{h \in H} h(D)\right| \geq|Y|-|H| \cdot|\partial|_{G}^{\max }(D),
$$

for $H$ as in the intersection inequality from 1.4 and finite dimensional subspaces $D$ in linear $\Gamma$-spaces.

Remarks. (a) There is a larger set of operators, denoted $G^{\star m} \supset G^{\cdot m}$, which is generated by $m$ operations of additions as well as compositions of linear maps $g \in G$. Namely, start from

$$
G^{\star 1}=\bigcup_{c \in \mathbb{F}} c G
$$

and then set

$$
G^{\star m}=\bigcup_{m_{1}+m_{2} \leq m}\left(\left(G^{\star m_{1}} \cdot G^{\star m_{2}}\right) \cup\left(G^{\star m_{1}}+G^{\star m_{2}}\right)\right) .
$$

Here again,

$$
I_{*}^{\max }(r ; G) \geq \frac{1}{m} I_{*}^{\max }\left(r ; G^{\star m}\right)
$$

and

$$
|\partial|_{\mu_{0}}(D) \geq \frac{1}{m}|\partial|_{\mu}(D),
$$

for a probability measure $\mu_{0}$ on $G$ depending on a given probability measure $\mu$ on $G^{\star m}$.

(b) Boundary defined with non-invertible operators. The $G$-boundary is supposed to measure the degree of (non-)invariance of a subspace $D$ under the operators $g \in G$ and if (some of) the $g \in G$ are non-injective on $D$ one may need to include into the $G$-boundary the kernels of these $g$.

1.7. $I_{*}$ for decaying functions. Let the underlying field $\mathbb{F}$ come with a norm, e.g., $\mathbb{F}$ equals the field of real, complex, or $p$-adic numbers, $\mathbb{R}, \mathbb{C}$ and $\mathbb{Q}_{p}$, respectively. Then every decaying $\mathbb{F}$-function $f$ on a countable set $X$ defines an indexing of $X$ by positive integers $i \in \mathbb{N}_{+}$, for which the sequence of norms $s(i)=\left\|f\left(x_{i}\right)\right\|$ is (non-strictly) monotone decreasing.

If all functions $f$ in a finite linear space $D$ of functions on a set $X$ (e.g. on $X=\Gamma$ ) decay super-exponentially, i.e., the corresponding sequences satisfy $C^{i} s(i) \rightarrow 0$ for all $C>0$ and $i \rightarrow \infty$, then one can replace (approximate) $D$ by a linear space $D_{0}$ of functions with finite supports on $X$ such that $\left|D_{0}\right|=|D|$ and $\left|\partial_{G}\left(D_{0}\right)\right|=\left|\partial_{G}(D)\right|$ for a given finite set $G$ of transformations of $X$. 
Sketch of the proof. The determinants of the $(k \times k)$-minors of the matrix corresponding to the system LIN in 1.5 are integers (exponentially) bounded by $|G|^{k}$. The solutions of the system LIN in the variables $f_{x}$, now indexed by $i \leftrightarrow x_{i}$, can be obtained by extending the solutions of the sub-systems for $f_{j \gamma}\left(x_{i}\right), j \leq j_{N} \rightarrow \infty$, and such extension amounts to solving some non-homogeneous system associated with LIN, where the non-homogeneity takes care of the $|D|=r$ condition by prescribing the values of the solutions (functions) at certain points in $X$. The rate of decay (as well as of growth) of such extensions cannot be (much) faster than $|G|^{j_{N}}$ and then the existence of a faster decaying solution implies that for a finitely supported one.

(The "rate of decay" on linear subspaces $D$ is seen with the help of suitable bases in $D$, where the superexponentiality is apparently needed. We leave the details to the reader, since we do not use such decaying solutions in this work).

Questions. Does the isoperimetric profile of the space of decaying (i.e., converging to zero at infinity) functions with values in an ultrametric field always equal (or, at least, is equivalent) to $I_{*}$ for functions with finite supports? Does a similar equality (or at least, equivalence) hold for the Hilbert spaces of square summable real- and complex-valued functions?

We shall prove the equality for the free Abelian groups and the equivalence for the non-virtually nilpotent polycyclic groups in 6.2.

Non-decaying functions, representations, finite groups, etc. We are mostly concerned in this article with the regular representation of an infinite group $\Gamma$ where we try to "linearize" combinatorics of finite subsets in $\Gamma$.

Other infinite dimensional, say irreducible, representations $L$ of $\Gamma$, e.g. realized by spaces of (non-decaying) functions on $\Gamma$, may have "good isoperimetry" for "a non-combinatorial reason", e.g., if they do not contain, in some weak sense, "bad" sub-representations.

Also $|\partial(D)| /|D|$ may be bounded from below in terms of the distance from $|D|$ to "bad" invariant subspaces, e.g., those of dimensions close to $|D|$, where many examples come from (families of) finite groups, such as $\operatorname{GL}\left(k ; \mathbb{F}_{p}\right)$ where suitable Følner functions, probably, behave "nicely" for $k, p \rightarrow \infty$.

\subsection{Non-amenability of groups and $I_{*}$-linearity theorems by Elek and Bartholdi.} A countable group is called non-amenable if it satisfies the linear isoperimetric inequality with some finite subset $G \subset \Gamma$ (where one may assume that $G$ generates $\Gamma$ if the group is finitely generated), i.e., if the function $I_{\circ}(r)$ is the maximal possible up to the asymptotic equivalence $I_{\circ}(r) \sim r$, which amounts here to $I_{\circ}(r) \geq c r$ for $c>0$; otherwise the group is called amenable.

The basic examples of non-amenable groups are the free groups on two or more generators and the groups containing such free groups as subgroups. Also, there 
are many non-amenable groups without free subgroups, e.g., non-amenable torsion groups.

On the other hand all solvable (e.g. Abelian groups) are amenable.

Remark. All known infinite amenable groups, in particular the groups of subexponential growth, either contain arbitrarily large finite subgroups or they have "many" commuting elements, but no general result of this kind is known. Here is a test question.

Let $Z\left(\gamma_{0}\right) \subset \Gamma$ for a given $\gamma_{0} \in \Gamma$ consist of those $\gamma \in \Gamma$, where some powers $\gamma_{0}^{m_{0}}$ and $\gamma^{m_{1}}$ commute. Does every infinite amenable group $\Gamma$ contain a non-identity element $\gamma_{0} \in \Gamma$ such that $\left|Z\left(\gamma_{0}\right) \cap G^{\cdot n}\right| / n \rightarrow \infty$ for $n \rightarrow \infty$, where $G \subset \Gamma$ is a finite generating subset?

An action of an algebra $A$ on a linear space $L$ is called non-amenable or $I_{*}$-linear, if its isoperimetric profile $I_{*}(r)$ defined with some finite subset of operators $G \subset A$ is equivalent to $r$. If $L$ equals the algebra itself then the algebra is called $I_{*}$-linear or non-amenable. (Unlike the group theoretic case, it is unclear what is the true property that divides algebras into "amenable" and "non-amenable" categories; the less obliging terminology seems appropriate.)

It is obvious that if $\Gamma$ is amenable then so is each of its linear actions and the converse is known in the following cases.

$\boldsymbol{I}_{*}\left(\boldsymbol{F}_{2}\right)$-linearity theorem. The group algebra of the free group $F_{2}$, and hence, of every group containing $F_{2}$ is $I_{*}$-linear for all fields $\mathbb{F}$. (Gabor Elek [6]; see 2.3 for a strengthening of this result.)

$l_{2}$-non-amenability theorem. The action of every non-amenable group $\Gamma$ on the complex Hilbert space $l_{2}(\Gamma)$ is $I_{*}$-linear. (Gabor Elek [8]; see 2.2 for the case of $l_{p}$, $p \neq 2$.)

$I_{*}$-linearity theorem. The group algebras of all non-amenable groups over all fields F are $I_{*}$-linear. (Laurent Bartholdi [2]; see 3.6 for a combinatorial rendition of Bartholdi's proof; if $\operatorname{char}(\mathbb{F})=0$, this follows from the $l_{2}$-theorem.)

Question. Is the action of a non-amenable group $\Gamma$ (e.g. for $\Gamma=F_{2}$ ) on the space of decaying $\mathbb{F}$-valued functions on $\Gamma$, where $\mathbb{F}$ is a normed field (e.g. the field of real numbers or the field of formal power series over a finite field), $I_{*}$-linear?

1.9. The growth functions $G_{\circ}$ and the Følner functions $F_{\circ}$ of groups and $F_{*}$ of algebras. The growth function $G_{\circ}(n), n=1,2, \ldots$, of a group $\Gamma$ relative to a given generating set $G \subset \Gamma$ is the number of elements in $\Gamma$ representable by the words of 
length $\leq n$ in the letters $g \in G$. That is, $G_{\circ}(n)=\left|G^{\cdot n}\right|$ for

$$
G^{\cdot n}:=\underbrace{G \cdot G \cdot \ldots \cdot G}_{n} \subset \Gamma .
$$

The growth function is at most exponential for finite sets $G$, since $\left|G^{\cdot n}\right| \leq|G|^{n}$.

The Folner function $F_{\circ}=F_{\circ}(n), n=1,2, \ldots$, of $(\Gamma, G)$ is equal, by definition, to the minimal cardinality of a (Følner) subset $Y_{n} \subset \Gamma$ such that $\left|\partial_{G}\left(Y_{n}\right)\right| \leq \frac{1}{n}|Y|$.

This can be expressed entirely in terms of $I_{\mathrm{o}}$ : define, for an arbitrary function $I$, the Folner function associated to $I$ by the rule:

the value of the function $F_{I}$ at $n$ equals the minimal $N$ such that $I(N) \leq N / n$, and observe that $F_{I}(n)=F_{\circ}(n ; \Gamma)$ for $I(r)=I_{\circ}(r ; \Gamma)$. In other words, $F_{I}$ is the inverse function to the minimal monotone increasing majorant $M(r)$ of the function $r / I(r)$. Thus, returning from $F$ back to $I$, gives us $r / M(r) \leq I(r)$ instead of $I(r)$, where the equality takes place if and only if $r / I(r)$ is monotone increasing. (We disregard the minor ambiguity which arises for non-strictly monotone functions.)

Unlike the isoperimetric profiles $I(r)$ that grow at most linearly and often are concave functions, the Følner functions of infinite groups grow faster than linearly and are rather convex than concave, where the rate of their growth translates into how slowly the corresponding isoperimetric profiles deviate from non-constant linear functions.

One may regard the map $Y \mapsto \partial Y$ as an "operator" on the "space of sets" with the "I $\mid$-norm" and think of the corresponding function $M(r)=\sup _{|Y| \leq r}|Y| /|\partial Y|$ as the "norm" of the "inverse operator" $\partial^{-1}$, where this "norm" is not a number but rather a function depending on $r=|Y|$ since $\partial$ is a "non-linear operator".

It is also helpful to think of the ratio $n=|Y| /|\partial(Y)|$ as a linear size (diameter) of $Y$ and of the Følner function as the volume (of the minimal $Y$ with given diameter) depending on the diameter.

It is clear that $F_{c I}(n)=F_{I}(c n)$ for all $c>0$. Thus, for example, the $G^{\cdot m_{-}}$ inequality $I_{\circ}(r ; \Gamma, G) \geq \frac{1}{m} I_{\circ}\left(r ; \Gamma, G^{\cdot m}\right.$ (see 1.4) translates to

$$
F_{\circ}(n ; \Gamma, G) \geq F_{\circ}\left(n / m ; \Gamma, G^{\cdot m}\right) .
$$

$(n / m)$,

and the (DIS)-inequality from 1.4 applied to the minimal $m$ for which $\left|G^{\cdot m}\right| \geq 2|Y|$, yields the

\section{Couhlon-Saloff-Coste isoperimetric inequality.}

$$
F_{\circ}(n ; \Gamma, G) \geq \frac{1}{2} G_{\circ}\left(\frac{n}{2} ; \Gamma, G\right) . \quad\left(F_{\circ} \succeq G_{\circ}\right)
$$

This inequality (that improves an earlier result by Varopoulos [28] with a rather elaborate proof exploiting random walks in groups) implies, for instance, that 
the groups of exponential growth have at least exponential Følner functions.

But unlike $G_{\circ}(n)$, the Følner functions $F_{\circ}(n)$ of some (amenable!) groups may grow (much) faster than exponentially as was conjectured by A. Vershik in the late 1960s and confirmed in [27] and [9]. (We shall meet such groups in the later sections.)

The useful notion of equivalence between two Følner functions is different from that for $I$ :

we write $F \prec F^{\prime}$ if $F(n) \leq F^{\prime}(C n)$ for some constant $C>0$, and $F \sim F^{\prime}$ if $F \prec F^{\prime}$ as well as $F^{\prime} \prec F$, and we apply the same notion of equivalence to the growth functions.

Examples. (a) The Coulon-Saloff-Coste inequality gives the correct asymptotic for $F_{\circ}^{\max }(n) \sim n^{k}$ for nilpotent (e.g. Abelian) groups (as was earlier proved by Pansu for the Heisenberg group and by Varopoulos in general).

Remark. There is a significant discrepancy between the lower bound $F_{\circ}^{\max }(n) \geq$ $\frac{1}{2} G_{\circ}\left(\frac{1}{2} n\right)$ and the available upper bounds. For instance, if $\Gamma=\mathbb{Z}^{k}$, then $G_{\circ}(n)=$ $\frac{1}{k !}(2 n)^{k}+o\left(n^{k}\right)$, while the (obvious) upper bound $F_{\circ}^{\max }(n) \leq n^{k}$ is provided by the $n$-cubes $C_{n} \subset Z^{k}$, where $\left|C_{n}\right|=n^{k}$ and $\left|\partial^{\max }\right|\left(C_{n}\right)=n^{k-1}$. (In fact, the cubes are the extremal sets in $Z^{k}$ and $F_{\circ}\left(n ; \mathbb{Z}^{k}\right)=n^{k}$, see 4.1).

Question. What is the asymptotic of $F_{\circ}\left(n ; \Gamma\left(k_{1}, k_{2}\right)\right)$ for the free nilpotent groups $\Gamma\left(k_{1}, k_{2}\right)$ on $k_{1}$ generators and of the nilpotency degree $k_{2}$ for $n, k_{1}, k_{2} \rightarrow \infty$ ?

(b) A group is non-amenable if and only if $F_{\circ}(n)=\infty$ for large $n$.

(c) $F_{\circ}(n ; \Gamma)$ is bounded if and only if $\Gamma$ is finite, where it equals $|\Gamma|$ for $n>|\Gamma|$, while infinite groups have $F_{\circ}(n) \succ n$ where the equivalence $F_{\circ}(n ; \Gamma) \sim n$ implies (by an easy argument) that the group is commensurable with $\mathbb{Z}$.

Folner functions $F^{\max }, F_{*}$, etc. Since the definition of $F_{\circ}$ makes sense for an arbitrary function $I(r)$ instead of $I_{\circ}$ one can define $F_{*}$ associated to the profiles of group algebras as well as the Følner functions for $|\partial|^{\max }$, in groups and linear spaces alike.

We shall introduce in 4.2 certain operations on functions, called $F \phi$ lner transforms, that correspond to some group theoretic constructions, e.g. Cartesian products and wreath products (see 9.1), and show that these particularly nicely behave on the class of what we call •-convex functions, where the notion of the •-convexity incorporates the Shannon inequality for the entropies of random variables.

The first instance where one sees the difference between $F_{*}$ and $F_{\circ}$ appears for finite groups: the Følner functions of the group algebras of finite groups $\Gamma$ are $F_{*}(n ; \Gamma)=1$ for all $n=1,2, \ldots$, since the 1 -dimensional space of constant functions has zero boundary. This leads (see 8.1) to examples of "very large" groups where $F_{*}(n ; \Gamma) \sim n$. 
On growth of algebras. Let $L$ be a linear space with a set $G$ of bijective operators acting on it, e.g. the group algebra of $\Gamma$. Then the growth function of $L, G$ is defined with some vector $l \in A$ as

$$
G_{*}(n, L, l, G):=\left|G^{\cdot n} * l\right|,
$$

where $G^{\cdot n} * l$ denotes the linear span of the orbit $G^{\cdot n}(l) \subset L$.

If the group algebra $A$ of $\Gamma$ generated by $G$ acts freely or without torsion on $L$, i.e., $a(l)=0$ only if $a=0$ and $l=0$, then this growth function equals that of $\Gamma$. In general, it may be smaller than $G_{\circ}(n ; \Gamma, G)$ and one may define $G_{*}^{\text {inf }}(n, L, G)$ as the infimum of $G_{*}(n ; L, l, G)$ over all $l \neq 0$ in $L$.

It is unknown (Kaplansky conjecture) if the freedom condition is satisfied by the group algebras of the groups without torsion and it is unclear how much $G_{*}(n ; L, l, G)$ may depend on $l$ in general.

Question. When is $G_{*}(n, L, l, G)$ asymptotically equivalent to $G_{*}^{\inf }(n, L, G)$ ?

Observe that the (obvious) linear algebraic counterpart of the (DIS)-inequality (see 1.4) reads as follows:

if an algebraic family $H$ of linear operators a acting on a linear space $X$ over an algebraically closed field has no zero vector in a given linear subspace $Y \subset X$, i.e., $a(y) \neq 0$ for $a \neq 0$ and $y \neq 0$, then

$$
|\partial|_{H}^{\max }(Y) \geq \operatorname{dim}(Y)(1-\operatorname{dim}(Y) / \operatorname{dim}(H)) .
$$

However, this does not match, at least not in an obvious way, the $\left(G^{* m}\right)$-inequality for the linear actions.

It remains unclear if there is a bound on the growth of the algebra by the Følner function, say for the torsion-free (i.e. without divisors of zero) algebras.

1.10. Multivariable Følner functions, $P_{\circ}$-functions and the Loomis-Whitney inequality. The multivariable Følner function $F_{\circ}\left(n_{i} ; X, G_{i}\right)$ for several sets of transformations $G_{i}$ acting on $X$ is defined as the maximal $N$ such that for every $Y \subset X$ with

$$
\left|\partial_{G_{i}}(Y)\right| \leq \frac{1}{n_{i}}|Y|
$$

one has

$$
|Y| \geq N
$$

and similarly one defines multivariable $F_{*}$ for linear actions.

These functions reduce to the ordinary $F_{\circ}^{\max _{i}}(n)$ and $F_{*}^{\max _{i}}(n)$ at $n_{1}=n_{2}=$ $\cdots=n$, where $\max _{i}$ refers to $|\partial|=\max _{i}\left|\partial_{G_{i}}\right|$. 
The $P_{\circ}$-function for orbit partitions. Let $X$ be acted upon by groups $\Gamma_{i}, i=$ $1,2, \ldots, k$, and denote by $\Gamma_{\circ}$ the intersection $\bigcap_{i} \Gamma_{i}$. Consider subsets $Y \subset X$ that have finite (or finite measure) images $Y / \Gamma_{\circ}$ in the quotient space $X / \Gamma_{\circ}$ and define $P_{\circ}\left(n_{i} ; X / \Gamma_{i}\right)$ as the minimal $N$ such that the inequalities

imply

$$
\left|Y / \Gamma_{i}\right| \leq \frac{1}{n_{i}}\left|Y / \Gamma_{\circ}\right|, \quad i=1,2, \ldots, k,
$$

$$
\left|Y / \Gamma_{\circ}\right| \geq N
$$

Observe that this $P_{\circ}$-function, unlike $I_{\circ}$ and/or $F_{\circ}$, depends only on the $\Gamma_{i}$ - orbit partition structure in $X$ rather than on the full $\Gamma$-structure in $X$.

If $\Gamma_{\circ}=\{\mathrm{id}\}$, while the $\Gamma_{i}$-orbits are all infinite, then $P_{\circ}\left(n_{i}\right) \leq F_{\circ}\left(n_{i}\right)$, since each infinite $\Gamma_{i}$ orbits that meets a finite subset $Y \subset X$ contributes a point to the $\Gamma_{i}$-boundary of $Y$.

Basic example: Loomis-Whitney theorem (see 5.7). The $P_{\mathrm{o}}$-function for the family of the $k$ partitions of $\mathbb{R}^{k}$ by the lines parallel to the $k$ coordinate axes is

$$
P_{\circ}\left(n_{i}\right)=\prod_{i} n_{i}
$$

In other words,

among all subsets $Y \subset \mathbb{R}^{k}$ with given measures of the projections to the $k$ coordinate hyperplanes, the maximal measure is achieved by the rectangular solids (and all subsets obtained from them by measurable transformations of $\mathbb{R}^{k}$ preserving the coordinate line partitions).

It follows that the multivariable Folner function of the free Abelian group $\mathbb{Z}^{k}$ with respect to its $k$ generators $g_{i} \in \mathbb{Z}^{k}$ (i.e., $G_{i}=\left\{g_{i}\right\}$ ) is

$$
F_{\circ}\left(n_{1}, n_{2}, \ldots, n_{k}\right)=n_{1} \cdot n_{2} \cdot \ldots \cdot n_{k} .
$$

Remark. We shall explain in 5.3 (this was pointed out to me by Noga Alon) how (LW) follows from the Shannon inequality for the entropies of random variables, where the latter, in the case of $\mathbb{R}^{k}$, is (essentially) equivalent to the logarithmic Sobolev inequality.

1.11. Filtered boundary $\mathscr{F} \boldsymbol{\partial}$ and $\boldsymbol{P}_{\boldsymbol{*}}$-functions. $F_{*}\left(n_{i}\right)$ and $P_{*}\left(n_{i}\right)$ for linear actions. The definition of the multivariable Følner function $F_{*}$ for groups $\Gamma_{i}$ acting on a linear space $L$ is identical to that of $F_{\circ}$, while $P_{*}$ needs a replacement of $|Y / \Gamma|$ by a suitable $\Gamma$-rank (dimension), denoted $|D: \Gamma|$ for linear subspaces $D \subset L$. 
There are several candidates for $P_{*}$ corresponding to different notions of the rank of a linear $\Gamma$-space. We start with a notion of a boundary associated to the Krull dimension.

$\mathcal{F}$ d-boundary. Given a group $\Gamma$ (or, more generally, an algebra $A$ ) acting on a linear space $L$, consider a $\Gamma$-invariant ascending filtration $\mathscr{F}=\left\{0=L_{0} \subset L_{1} \subset\right.$ $\left.L_{2} \subset \cdots \subset L\right\}$. Then, for a given linear subspace $D \subset L$, consider the subspaces $D_{i}=D \cap L_{i}$ and let $\partial_{\mathcal{F}}(D)$ denote the number of those $i=1,2, \ldots$ for which the subspace $D_{i} / D_{i-1} \subset L_{i} / L_{i-1}$ is not $\Gamma$-invariant. Clearly, this boundary minorizes the $\Sigma$-boundary $\partial_{\Sigma H}(D)$ for an arbitrary generating subset $H$ in $\Gamma$.

Then we define

$$
\mathcal{F} \partial_{\Gamma}(D)=\sup _{\mathscr{F}} \partial_{\mathscr{F}}(D)
$$

for all $\Gamma$-invariant filtrations $\mathscr{F}$ in $L$ and, given groups $\Gamma_{i}$ acting on $L$, one defines the "filtered" isoperimetric profile associated to the boundaries $\left|\mathcal{F} \partial_{\Gamma_{i}}(D)\right|$ and the corresponding multivariable Følner function. (The corresponding boundary in the combinatorial framework of $\Gamma$ acting on $X$ counts the number of the $\Gamma$-orbits which meet a $Y \subset X$ and which are not contained in $Y$.)

Let the group $\Gamma$ be amenable and $\mathscr{H}$ be an exhaustion by an increasing sequence of (Følner) subsets $H_{j} \subset \Gamma$ with $\left|\partial H_{j}\right| /\left|H_{j}\right| \rightarrow \infty$. If two finite dimensional subspaces $D_{1}, D_{2} \subset L$ have equal $\Gamma$-spans $\Gamma * D_{1,2} \subset L$, then their $H_{j}$-spans $H_{j} * D_{1,2}$ have the same asymptotic growth,

$$
\left|H_{j} * D_{1}\right| /\left|H_{j} * D_{2}\right| \rightarrow 1
$$

for $j \rightarrow \infty$.

On the other hand, if $\Gamma * D$ admits a $\Gamma$-invariant filtration $L_{i}$ where $L_{i} / L_{i-1}$ is $\Gamma$-generated by a $k_{i}$-dimensional subspace $\underline{D}_{i} \subset L_{i} / L_{i-1}$ for all $i=1,2, \ldots$, then

$$
\limsup _{j \rightarrow \infty}\left|H_{j} * D\right| /\left|H_{j}\right| \leq \sum_{i} k_{i} .
$$

Furthermore, if the group $\Gamma$ is infinite, while $|D|<\infty$, then the filtration $\mathscr{F}$ obtained by taking the $\Gamma$-span of a filtration $0=D_{0} \subset D_{1} \subset D_{2} \subset \cdots \subset D$ with $\left|D_{i} / D_{i-1}\right|=1$ satisfies

$$
\left|\mathcal{F} \partial_{\Gamma}(D)\right| \geq \limsup _{j \rightarrow \infty}\left|H_{j} * D\right| /\left|H_{j}\right| .
$$

All this motivates the definition of the asymptotic rank (dimension):

$$
|D: \Gamma|_{\mathscr{H}}=|D: \Gamma|_{\left\{H_{j}\right\}}=\limsup _{j \rightarrow \infty}\left|D_{j}\right| /\left|H_{j}\right|,
$$

where $|D|=\operatorname{rank}(D)$ and $\left|H_{j}\right|=\operatorname{card}\left(H_{j}\right)$. Then one defines

$$
|D: \Gamma|=\sup _{\mathscr{H}}|D: \Gamma|_{\mathscr{H}}
$$


and $P_{*}\left(n_{i} ; \Gamma_{i}\right)=P_{*}^{\text {sup }}\left(n_{i} ; \Gamma_{i}\right)$ with $\Gamma_{i} \subset \Gamma$ as in the case of $P_{\circ}$, but now with $\left|D: \Gamma_{i}\right|$ instead of $\left|Y / \Gamma_{i}\right|$.

Remark. Instead of "sup lim sup" one may use other "generalized limits" that give non-ambiguous definitions; see [8], [6], [14]. For example, one may consider $\liminf _{|H| \rightarrow \infty}|H * D| /|H|$ over all finite subsets $H \subset \Gamma$, where this leads to a meaningful (albeit not apparently useful) definition of $|D: \Gamma|$ for all not necessarily amenable groups $\Gamma$.

If $H \subset \Gamma$ is connected in the $G$-Cayley graph $\Gamma$ for a generating set $G \subset \Gamma$, then $|H * D| \leq|\partial|_{G}^{\max }(D)|\cdot| H \mid ;$ therefore,

$$
P_{*}\left(n_{i} ; \Gamma_{i}\right) \leq F_{*}\left(n_{i} ; \Gamma_{i}, G_{i}\right)
$$

for infinite $\Gamma_{i}$ and arbitrary finite generating subsets $G_{i} \subset \Gamma_{i}$.

Examples. (a) If $\Gamma=\mathbb{Z}^{k}$, then (obviously) $|D: \Gamma|$ equals the dimension of the linear span of $D$ over the field of rational functions in $k$ variables, which is the field of fractions of the group ring of $\mathbb{Z}^{k}$.

(b) If the action of $\Gamma$ on $L$ is free, i.e., no non-trivial finite linear combination $\sum_{\gamma} c_{\gamma}(a)$ vanishes, e.g., if $L$ equals $A$, the group algebra of $\Gamma$ over some field, where $A$ has no divisors of zero, then, obviously, $\left|D_{i}\right| \geq\left|H_{i}\right|$ and thus $|D: \Gamma| \geq 1$ for all non-zero $D \subset A$.

$l_{2}$-rank and $P_{*}^{l_{2}}$. If $L$ equals the Hilbert space of $l_{2}$-functions on an $X$ with a free action of $\Gamma$, then an alternative to the asymptotic rank is furnished by the von Neumann $l_{2}$-dimension $|D: \Gamma|_{l_{2}}$ defined for all closed $\Gamma$-invariant subspaces; if $D \subset L$ is not $\Gamma$-invariant, the notion of $l_{2}$-dimension applies to the $\Gamma$-span $\Gamma * D$ of $D$, which is the minimal closed $\Gamma$-invariant subspace containing $D$,

$$
|D: \Gamma|=|D: \Gamma|_{l_{2}}:=|\Gamma * D: \Gamma|_{l_{2}} .
$$

Clearly, $|D: \Gamma|_{l_{2}} \leq|D: \Gamma|_{\left\{H_{j}\right\}}$, whenever the two ranks are simultaneously defined. Furthermore, if $D$ has a finite support in $X$, then (this is also well known and obvious) $|D: \Gamma|_{l_{2}}=|D: \Gamma|_{\left\{H_{j}\right\}}$ for an arbitrary Følner exhaustion $H_{j}$. (Thus, one does not have to worry here about the existence of the limit in the definition of $\left.\left|D: \Gamma_{i}\right|.\right)$

If one tries to straightforwardly define the $P_{*}=P_{*}^{l_{2}}$-function with $|D: \Gamma|_{l_{2}}$, one gets it identically zero already for the coordinate subgroups $\Gamma_{i}=\mathbb{Z}, i=1,2, \ldots, k$, acting on $\Gamma=\mathbb{Z}^{k}$, but, whatever the definition of $P_{*}^{l_{2}}$, it depends on the Hilbert structure of $L$, while the above $P_{*}$ needs only the linear algebraic data and $P_{*}^{l_{2}} \leq P_{*}^{\text {inf }}$ whenever the two $P$-functions are simultaneously defined. Furthermore, if the space $D$ of functions on $X$ in the definition of $P_{*}^{l_{2}}$ has finite support, then $P_{*}^{l_{2}}=P_{*}^{\text {inf }}=$ 
$P_{*}^{\text {sup }}$, where "inf" and "sup" refer to the "inf lim inf" and "sup lim sup" definitions of the asymptotic rank.

Comparison between the graph metrics and the lattices of subsets and of linear subspaces. The lattice of the finite subsets $Y$ in a set $X$ is fully determined by the marked edge graph of the $X$-cube $Z=2^{X}$ of all finite subsets in $X$, where the vertices correspond to the finite subsets $Y \subset X$ and where one marks the vertex corresponding to the empty set. For example the distance between two subsets equals one half of the minimal edge length of the paths between the corresponding vertices.

Similarly, the lattice of finite dimensional linear subspaces $D$ in a linear space $L$ is determined by the 1-skeleton of the corresponding spherical building that is the graph, where the vertices correspond to the finite dimensional linear subspaces $D \subset X$, where the two vertices $D_{1}$ and $D_{2}$ are joined by an edge if and only if one of the two subspaces contains the other with codimension 1 , and where one marks the vertex corresponding to the 0 -space.

The evaluation of $P_{*}\left(n_{i}\right)$ as well as of the isoperimetric profile $I_{*}(r ; \Gamma, G)$ may be more difficult than that of their combinatorial o-counterparts (essentially) because the lattice of linear subspaces is not distributive: the intersection of a $D$ with $\operatorname{span}\left(D_{1}, D_{2}\right)$ can be strictly greater than the span of the intersections $D \cap D_{1}$ and $D \cap D_{2}$ (while $Y \cap\left(Y_{1} \cup Y_{2}\right)=\left(Y \cap Y_{1}\right) \cup\left(Y \cap Y_{2}\right)$ for all subsets of an $\left.X\right)$.

\subsection{Summary of results}

1. We shall prove in 2.2 , generalizing Elek's $l_{2}$-theorem, that

the action of every non-amenable group $\Gamma$ on the space $l_{p}(\Gamma)$ of $p$-summable real functions is $I_{*}$-linear for all $1 \leq p<\infty$.

We define in 2.3 a class of groups $\Gamma$, which includes the free groups $F_{k}$ for $k \geq 2$ and many (but not all) non-amenable groups that contain no $F_{2}$, such that their group algebras are $\mathcal{F}$-non-amenable (or $I_{\mathcal{F}-\text {-linear) }}$ in the following sense.

There exists a finite family of subgroups $\Gamma_{i} \subset \Gamma$ in each group $\Gamma$ from this class such that every linear subspace $D$ in the group algebra of $\Gamma$ over every field $\mathbb{F}$ has

$$
\max _{i}\left|\mathcal{F} \partial_{\Gamma_{i}}(D)\right| \geq \varepsilon|D|
$$

for some $\varepsilon=\varepsilon(\Gamma)>0$.

2. We show in 3.2 (elaborating upon a remark by Dima Grigorev who pointed out to me how the polynomial Brunn-Minkowski inequality reduces to the combinatorial one with an order on the set of monomials) that

if $\Gamma$ admits a left invariant order, then the linear algebraic profile equals the combinatorial one,

$$
I_{*}(r ; \Gamma)=I_{\circ}(r ; \Gamma),
$$


and prove some related results.

3. We combine in 6.2 the Coulon-Saloff-Coste argument with the $l_{2}$-Loomis-Whitney inequality and prove, for example, that

the Folner function $F_{*}(n)$ of the Hilbert space $l_{2}(\Gamma)$ of square summable functions on every polycyclic non-virtually nilpotent group $\Gamma$ has exponential growth.

(For the spaces of functions with finite supports this follows directly from the CoulhonSaloff-Coste inequality and 2.)

4. We shall apply the entropic formalism (which mimics the martingale method in isoperimetry) to the group algebras of normal extensions and thus prove, for example, that

Grigorchuk groups (which may have subexponential growth $G_{\circ} \prec \exp \left(n^{\alpha}\right)$ for $0<\alpha<1$ and may be pure torsion) satisfy

$$
F_{*}(n) \succ \exp \left(n^{\beta}\right) \text { for some } \beta>0
$$

(see 7.1,7.2). Also we prove in 7.2 that

the (twice or more) iterated wreath products $\Gamma$ of infinite groups, e.g. of ( pure torsion amenable) Aleshin-Grigorchuk groups, have

$$
F_{*}(n) \succ \exp (\exp (n)) .
$$

5. We shall exhibit in 8.1, 8.2 a class of amenable groups $\Gamma$, where the combinatorial $F \phi$ lner functions $F_{\circ}(n ; \Gamma)$ may grow arbitrarily fast

(these $\Gamma$ are extensions of locally finite groups as in the last remark in Section 3 of [9]) and, yet,

all these $\Gamma$ have bounded linear algebraic profiles,

$$
I_{*}(r ; \Gamma) \leq \mathrm{const}=\operatorname{const}(\Gamma) .
$$

On the other hand we produce

orderable amenable groups $\Gamma$ where both combinatorial and linear algebraic Følner functions grow arbitrarily fast.

(These $\Gamma$ are extensions of locally nilpotent groups rather than of locally finite groups that were suggested in [9]. Possibly, one can also render orderable the construction of groups with subexponential growth from [10] and make not only $F_{\mathrm{o}}$, as it is done in [10], but also $F_{*}$ grow arbitrarily fast.)

6. We present in 9.1-9.3 a translation of the combinatorial argument from [9] to the language of partitions, which improves the lower bounds from [9] on Følner functions of the iterated wreath products and related classes of groups. 
7. We shall prove in 10.1-10.3 some Brunn-Minkovski type inequalities for discrete groups and their group algebras.

Our presentation is self-contained and a significant part of the paper is expository.

Acknowledgment. What follows is an outgrowth of a section in my (still unfinished) paper [16] turned into a separate article by a suggestion by Slava Grigorchuk. A part of this paper was written during my visit to the Mathematics Department of the Northwestern University in a stimulating and hospitable atmosphere. Also I want to thank Tullio Ceccherini-Silberstein, Christophe Pittet and the anonymous referees who generously spent their time in reading the paper, pointed out a variety of errors and made many useful suggestions.

\section{Non-amenability of groups and strengthened non-amenability of group algebras}

\section{1. $l_{2}$-non-amenability of the complex group algebras of non-amenable groups}

Elek non-amenability theorem. If $\Gamma$ is non-amenable, then the isoperimetric profile of the group algebra of $\Gamma$ over $\mathbb{F}=\mathbb{C}$, and, hence over any field of characteristic zero, is linear:

$$
\operatorname{rank}(G * D)-\operatorname{rank}(D) \geq \varepsilon \cdot \operatorname{rank}(D)
$$

for all $G \subset \Gamma$ generating $\Gamma$, all finite dimensional linear subspaces $D \subset A$ and some constant $\varepsilon=\varepsilon(\Gamma, G)>0$.

This is shown in [8] in the following stronger form.

$\boldsymbol{l}_{\mathbf{2}}$-theorem. Let $\Gamma$ be a non-amenable group and $G \subset \Gamma$ be a finite generating set. Then there is a positive constant $\varepsilon$ such that for every $N$-dimensional linear subspace $D$ in the complex Hilbert space $l_{2}(\Gamma)$ there exists $\gamma \in G$ such that $\operatorname{dim}(\gamma(D) \cap D) \leq$ $(1-\varepsilon) \operatorname{dim}(D)$, where $\gamma(D)$ refers to the left action of $\Gamma$ on $l_{2}(\Gamma)$.

Proof. If $\Gamma$ is non-amenable, then, according to one of the definitions of (non-)amenability,

there is no almost invariant probability measure on $\Gamma$ : every real positive $l_{1}$-function $f$ on $\Gamma$ satisfies

$$
\max _{\gamma \in G}\|\gamma(f)-f\|_{l_{1}} \geq\left\|\varepsilon_{0} f(\gamma)\right\|_{l_{1}}
$$

for some positive $\varepsilon_{0}$ depending on $\Gamma$ and $G$. 
Denote by $e_{\gamma} \in l_{2}=l_{2}(\Gamma)$ for $\gamma \in \Gamma$ the (delta) function that equals 1 at $\gamma$ and vanishes at all other points in $\Gamma$, and let $f_{D}=f_{D}(\gamma)$ denote the square of the $l_{2}$-norm of the orthogonal projection of $e_{\gamma}$ to $D$ regarded as a function on $\Gamma$.

Since $e_{\gamma} \in l_{2}=l_{2}(\Gamma)$ for all $\gamma \in \Gamma$ make an orthonormal basis in $l_{2}$, one has $\left\|f_{D}\right\|_{l_{1}}=\operatorname{dim}(D)$ for all finite dimensional $D \in l_{2}$ and $\left\|f_{D}-f_{D^{\prime}}\right\|_{l_{1}} \leq$ $\operatorname{dim}\left(\left(D+D^{\prime}\right) /\left(D \cap D^{\prime}\right)\right)$. In fact, if a subspace $D_{+} \subset l_{2}$ splits into the orthogonal sum of $D_{1}$ and $D_{2}$ then $f_{D_{+}}=f_{D_{1}}+f_{D_{2}}$ by Pythagorean theorem.

Therefore, $\left\|\gamma\left(f_{D}\right)-f_{D}\right\|_{l_{1}} \leq 2 \operatorname{dim}((D+\gamma(D)) / D)$ for all $\gamma \in \Gamma$, and the proof follows.

2.2. Geometric proof that applies to $\boldsymbol{l}_{\boldsymbol{p}}(\boldsymbol{\Gamma})$. Given a finite dimensional Banach space $D$ we denote by $\mu_{D}$ the normalized measure on the unit sphere $S_{D}$ of $D$ induced from the Lebesgue measure on $D$ : the measure of a subset $U \subset S_{D}$ equals the measure of the cone over $U$ in the unit ball in $D$ divided by the Lebesgue measure of this ball.

Given two finite dimensional subspaces in a Banach space $(L,\|\cdot\|)$, say $D \subset L$ and $D^{\prime} \subset L$, we denote by $\mu$ and $\mu^{\prime}$ the push-forwards of the measures $\mu_{D}$ and $\mu_{D^{\prime}}$ to the unit sphere $S_{L} \supset S_{D}, S_{D^{\prime}}$ and consider all Borel maps $\xi$ from $S_{D} \subset S_{L}$ to $S_{D^{\prime}} \subset S_{L}$ sending $\mu$ to $\mu^{\prime}$.

Define the distance between the two measures by

$$
\delta\left(\mu, \mu^{\prime}\right)=\inf _{\xi} \int_{S_{D}}\|s-\xi(s)\| d \mu(s) .
$$

Say that $L$ is asymptotically $M$ (easure) $D$ (imension)-consistent if for every two sequences of finite dimensional subspaces with dimensions converging to $\infty$ for $i \rightarrow \infty$, say $E_{i} \subset L$ and $E_{i}^{\prime} \subset E$, the convergence $\operatorname{dim}\left(E_{i}\right) / \operatorname{dim}\left(E_{i} \cap E_{i}^{\prime}\right) \rightarrow 1$ for $i \rightarrow \infty$ implies the convergence $\delta\left(\mu_{i}, \mu_{i}^{\prime}\right) \rightarrow 0$.

This consistency is classically known for $l_{2}$ (by the Maxwell distribution law for the ideal gas) and it is equally obvious for all uniformly convex Banach spaces, such as $l_{p}$ for $1<p<\infty$, that these spaces are MD-consistent. (See [18], for instance.)

If an isometric group action on an asymptotically MD-consistent space $L$ has an almost fixed point on the Grassmannian $\mathcal{E}^{*}(L)$ with the normalized metric $|\cdot-\cdot|$ (see 1.5) then there exists an almost invariant probability measure on the unit sphere $S$ in $L$ for the metric $\delta$.

Another relevant property of $l_{p}(\Gamma)$ is the existence of a $\Gamma$-equivariant Lipschitz map (playing the role of the domination map in 3.1,3.2) from the unit sphere $S_{l_{p}} \subset$ $l_{2}(\Gamma)$ to the space $S_{1}^{+} \subset l_{1}$ of the probability measures on $\Gamma$ : each $l_{p}$-function $f(\gamma)$ goes to $f^{p}(\gamma)$, where this map (a radial projection in the logarithmic coordinates, compare (c) in 5.4) is invariant under all permutations of the basic vectors.

Thus every almost invariant measure $\mu$ on $S_{l_{p}}$ gives us such a measure $\mu_{*}$ on the positive "quadrant" $S_{l_{1}}^{+} \subset S_{l_{1}}$, that is, the space of probability measures on $\Gamma$. Since 
$S_{l_{1}}^{+}$is a closed convex subset in $l_{1}(\Gamma) \supset S_{l_{1}}$, each measure on $S_{l_{1}}^{+}$has a center of mass and this center, say $\mu_{*} \in S_{l_{1}}^{+}$, is a probability measure on $\Gamma$ itself. Clearly, $\mu_{*}$ is almost invariant for the $l_{1}$-metric whenever the measure is $\mu$ almost invariant for $\delta$.

Remark. The first (Pythagorean) proof of Elek's theorem shows that there is no Hilbert-Schmidt operator on $l_{2}(\Gamma)$ that almost commutes with the action of $\Gamma$, while the geometric argument establishes the "no almost fixed point" property on the Grassmannian of finite dimensional linear subspaces with the metric induced by the (measure transportation) Monge-Kantorovich metric.

Questions. Let $X$ be a countable set, $L$ some linear space of $\mathbb{F}$-valued functions on $X$ invariant under the group $\Gamma$ of transformations of $X$ (e.g. the group of all bijective transformations) and let $\mathrm{Gr}_{d}$ be the space of all $d$-dimensional subspaces $D \subset L$ with the metric $\left|D_{1}-D_{2}\right|:=\frac{1}{d} \mid D_{1} /\left(D_{1} \cap D_{2} \mid\right)$.

What is the infimum of the Lipschitz constants of the $\Gamma$-equivariant maps from $\mathrm{Gr}_{d}$ to the space $\mathcal{P}(X)$ of the probability measures on $X$ for specific "natural" spaces $L$ ?

What are the Lipschitz constants for equivariant maps between the Grassmannians $\mathrm{Gr}_{d}$ associated to different spaces $L$ (with, possibly, different $\mathbb{F}$ ) of functions on $X$ ?

Such maps to $\mathcal{P}(X)$ play the key role in the proof of the $I_{*}$-linearity theorem by Bartholdi; we shall present a version of his construction in 3.6.

Two comments made by an anonymous referee. (1) It might be interesting to (further, M. G.) geometrize the study of linear isoperimetric profiles à la Voisculescu, so that one considers not the linear span of translates of a finite dimensional subspace but rather the smallest dimension of a subspace approximately containing all of these translates granted that the ambient space is equipped with a norm. This would seem to tie in with Bekka's notion of amenability for unitary representations and it would provide a useful analytic tool for the study of isoperimetry in spaces other than $l_{2}$ and group algebras while at the same time directly connecting to the linear theory in these special cases, which are the primary objects of study in this article.

(2) The approximate invariance of a Følner set can be expressed in a dual way via approximately even coverings with a certain multiplicity. This leads to a recursive extraction procedure of the quasitiling theorem of Ornstein and Weiss according to which the Følner set can be approximately tiled by translates of finitely many tiles at different scales. Might the type of isoperometry technology elaborated upon in the paper be used, via some duality principle, to study the problem of the number of different scales required to quasitile Følner sets in various groups (about which little seems to be known). 


\section{3. $F$-non-amenability of the group algebras of groups with acyclic families of subgroups}

(A) Topological argument for non-amenability of $\boldsymbol{F}_{\boldsymbol{k}}$. The free group $\Gamma=F_{k}$ on $k \geq 2$ generators freely acts on the infinite regular $2 k$-valent tree $T_{k}$. Every finite subtree with the vertex set $Y \subset X$ consisting of $r$ vertices $y_{i}, i=1,2, \ldots, r$, of degrees $d_{i}$ satisfies, by the Euler-Poincaré formula,

$$
r-\frac{1}{2} \sum_{i} d_{i}=1
$$

Hence

$$
\left|\partial^{\text {joint }}(Y)\right|=\sum_{i}\left(2 k-d_{i}\right)=2 r k-2 r+2
$$

and

$$
I_{\circ}(r ; \Gamma)=r(2 k-2)+2 .
$$

It follows that $F_{k}$ for $k \geq 2$ is non-amenable and, by the extension property, that the groups containing an $F_{2}$ as a subgroup are non-amenable.

We shall return to the topological aspects of isoperimetry in [16], but now let us indicate another approach for estimating $I_{\circ}$ that applies, for example, to Burnside groups.

(B) Slicing argument. Look at how the orbits (cosets) of the $k$ generating cyclic subgroups $\Gamma_{i}$ in $\Gamma=F_{k}$ meet and "slice" a finite subset $Y_{0} \subset \Gamma$, say for $\Gamma=F_{2}$.

Since every finite (sub)tree contains at least one leaf (end-vertex) $y$, there exists a subgroup among the generating two such that some of its orbits (cosets) in $\Gamma$ meet $Y_{0}$ only at $y$. (In general, there are $k-1$ of such subgroups at every leaf.) We remove from $Y$ the intersection $S_{1}=S_{1}\left(Y_{0}\right)$ of such an orbit with $Y_{0}$, then remove a similar $S_{2}=S_{2}\left(Y_{1}\right)$ from $Y_{1}=Y_{0} \backslash S_{1}$, etc., until we exhaust all of $Y_{0}$. Thus we obtain $\left|Y_{0}\right|$ orbits non-trivially meeting $Y_{0}$; therefore there are at least $\left|Y_{0}\right| / 2$ orbits of one of these subgroups, say of $\Lambda \subset \Gamma$, that meet $Y_{0}$ and

$$
\left|\partial_{\Lambda}(Y)\right| \geq \frac{1}{2}|Y|
$$

thus the max-boundary of $Y$ in $\Gamma$ is also bounded from below by $\frac{1}{2}|Y|$. (More generally, if $k \geq 2$, then $|\partial|^{\max }(Y) \geq \frac{k-1}{k}|Y|$ by the slicing argument.)

This may seem no better than the above bound for $\left|\partial^{\text {joint }}(Y)\right|$, but the slicing argument itself carries more power. For example, it implies the following. 
(C) $\mathscr{F}$ and $\boldsymbol{P}_{*}$-non-amenabilty of the group algebra of $\boldsymbol{\Gamma}=\boldsymbol{F}_{\mathbf{2}}$. Let $D_{0}$ be a linear space of functions on $\Gamma$ with finite support $Y_{0}=\operatorname{supp}\left(D_{0}\right)$. Then the restriction homomorphism $h_{1}$ from $D_{0}$ to (the space of linear functions on) the above slice $S_{0} \subset Y_{0}$ clearly has rank 1 .

Let $D_{1}=\operatorname{ker}\left(h_{1}\right) \subset D_{0}$ and let $h_{2}$ be a similar homomorphism from $D_{1}$ to such a slice $S_{2} \subset Y_{1}=\operatorname{supp}\left(D_{1}\right)$, etc. Thus we obtain for one of these two cyclic subgroups, say for $\Lambda \subset \Gamma$,

an increasing chain of $\Lambda$-invariant subsets, $Z_{0} \subset Z_{1} \subset \cdots \subset Z_{n}$ for $n \geq$ $\left|D_{0}\right| / 2$, such that the kernels $D_{i}^{\prime} \subset D_{0}$ of the restriction homomorphisms from $D_{0}$ to (the spaces of functions on) $Z_{i}^{\prime}$ strictly(!) decrease with $i$.

It follows that the filtered boundary and the asymptotic rank (see 1.11) satisfy

$$
|D: \Lambda| \geq\left|\mathcal{F} \partial_{\Lambda}(D)\right| \geq n \geq\left|D_{0}\right| / 2 .
$$

Indeed, take a splitting $D_{0}=\oplus_{i} \Delta_{i}$ compatible with the filtration $D_{i}^{\prime}$ of $D_{0}$ and observe that the spans $\operatorname{span}\left(\Lambda\left(\Delta_{i}\right)\right)$ are linearly independent, since $\operatorname{supp}(\Lambda(D))=$ $\Lambda(\operatorname{supp}(D))$ for all linear spaces $D$ of functions on $\Gamma$.

This implies the required linear lower bound on $\left|\mathcal{F} \partial_{\Lambda}(D)\right|$ and so such a bound on the asymptotic rank $|D: \Lambda|$, which is the dimension of the span of $D$ over the field of fractions of the (commutative!) group algebra of $\Lambda$.

More generally, one has the following.

(D) $\mathcal{F}$-non-amenability in the presence of divergent families of subgroups. Let $X$ be $a \Gamma$-space and let $\Gamma_{i} \subset \Gamma, i=1,2, \ldots, k<\infty$, be a 1-divergent family of subgroups, which means, by definition, that for every finite subset $Y \subset X$ there is an orbit $S \subset X$ with $|S|>k$ of some $\Gamma_{i}$ which satisfies

$$
|S \cap Y|=1 .
$$

Then the action of $\Gamma$ on the space of $\mathbb{F}$-valued functions on $X$ with finite supports is $I_{\mathscr{F}}$-linear for all fields $\mathbb{F}$.

Proof. There is an $i$ such that by successively making the function from $D$ vanish on $\Gamma_{i}$-orbits $S_{m}$ we obtain a strictly descending chain $D_{m} \subset D$ of length $\geq|D| / k$, where there are at least $|D| / k^{2}$ subspaces $D_{m}$ with $\left|D_{m-1} / D_{m}\right|<\left|S_{m}\right|$. Since every $D_{m-1}$ contains a function supported on a single point in $S_{m}$, the corresponding quotients $\left|D_{m-1} / D_{m}\right|$ are not $\Gamma_{i}$-invariant.

(E) Corollary (Acyclicity $\Rightarrow I_{\mathcal{F}}$-linearity). Let $\Gamma$ admit an infinite sequence of $\Gamma_{j}, j=1,2, \ldots$, with cardinalities $\left|\Gamma_{j}\right|>k$, where $k$ equals the number of different subgroups among $\Gamma_{j}$, such that

$$
\gamma_{j_{1}} \cdot \gamma_{j_{1}+1} \ldots \gamma_{j_{2}-1} \cdot \gamma_{j_{2}} \neq \mathrm{id}
$$


for $\mathrm{id} \neq \gamma_{j} \in \Gamma_{j}$ and all $j_{1} \leq j_{2}$. (Such sequences are called acyclic.) Then the group algebra of $\Gamma$ over any field is $I_{\mathcal{F}}$-linear.

Proof. If a subset $Y \subset \Gamma$ has $|Y \cap S| \geq 2$ for all orbits (cosets) of all $\Gamma_{j}$ that meet $Y$, then $Y$ contains infinitely many $\gamma$ of the form $\gamma_{0}, \gamma_{j_{0}} \cdot \gamma_{j_{1}}, \gamma_{j_{0}} \cdot \gamma_{j_{1}} \cdot \gamma_{j_{1}+1}, \ldots$ for $\gamma_{0} \in Y$ and $\gamma_{j} \in \Gamma_{j}$ for $j \geq 1$.

Examples. (a) If $\Gamma$ is equal to a free product, $\Gamma=\Gamma_{1} * \Gamma_{2}$, then the sequence $\Gamma_{1}, \Gamma_{2}, \Gamma_{1}, \Gamma_{2}, \ldots$ is acyclic and the above applies to infinite $\Gamma_{1}$ and $\Gamma_{2}$. (This argument yields the non-amenabilty of $\Gamma$ itself for $\left|\Gamma_{1}\right| \geq 2$ and $\left|\Gamma_{2}\right| \geq 3$ as everybody knows.)

(b) Consider a square-free sequence $i_{j} \in I=\{1,2, \ldots, k\}, k \geq 3$, i.e., where there are no subsequences $i_{j}, i_{j+1}, \ldots, i_{j+2 l}$ with $i_{j+s}=i_{j+l+s}$ for $s=0,1, \ldots l$. Then take a free product $\Gamma_{0}=\Gamma_{1} * \Gamma_{2} * \cdots * \Gamma_{k}$, enumerate all $\gamma \in \Gamma$ by $m=1,2, \ldots$, and add to $\Gamma$ torsion relations $\gamma_{m}^{n_{m}}=$ id one after another, omitting those where the corresponding $\gamma_{m}$ was already torsion in the preceding factor group.

The hyperbolic small cancellation tells us that the groups $\Gamma_{i}$ inject into the resulting factor group $\Gamma$ for torsion-free $\Gamma_{i}$ and fast growing $n_{m}$, and that the sequence of subgroups $\Gamma_{i_{j}}$ is acyclic in the above sense for square-free sequences $i_{j}$.

Moreover (this was explained to me by Azer Akhmedov, Ashot Minasyan and Dima Sonkin), this property is known for free Burnside p-torsion groups $\Gamma_{i}$ and all $n_{m}=p$ with $p$ large enough.

The Burnside groups $B(p, 2)$ with 2 generators contain $B(p, k)$ for all $k \geq 6$; thus, they contain the $p$-torsion quotients of $B(p, 2) * B(p, 2) * B(p, 2)$. Therefore we can state:

\section{(F) The groups $B(p, k)$ are $I_{\mathscr{F}}$-linear for all sufficiently large $p$ and $k \geq 2$.}

Remarks. (a) Random quotients $\Gamma$ of all resilient groups (see [15]), e.g. Burnside groups, are not acyclic in the above sense (where some of them are "Olshanskii monsters" with all their proper subgroups isomorphic to $\mathbb{Z}_{p}$, as was pointed out to me by Dima Sonkin, but being a monster does not by itself exclude acyclicity). It is unclear which of them possess some "strengthened non-amenability" controlled by the combinatorics of orbit partitions and/or of invariant filtrations in the linear algebraic setting. (The ordinary non-amenability of such groups follows by the Grigorchuk criterion and can also be derived from Kazhdan's $T$-property in some cases.)

(b) We emphasized the $I_{\mathcal{F}}$-linearity $(=\mathscr{F}$-non-amenability) rather than the (weaker property of) $I_{*}$-linearity (= linear algebraic non-amenability), since the latter is valid by the Elek-Bartholdi theorem for all non-amenable groups $\Gamma$. 


\section{Isoperimetry in the group algebras of ordered groups}

We show in this section that the isoperimetric inequalities in the (left) orderable groups $\Gamma$ pass from subsets in $\Gamma$ to linear subspaces in the group algebra of $\Gamma$ over any field.

3.1. Triangular systems and bases. Let $X=(X, \preceq)$ be an ordered set, e.g., $X=\{1,2, \ldots, n\}$, take a function $f$ on $X$ with values in some field (any set with a distinguished element called 0 will do for the following definition) and denote by $x_{f}^{\min } \in X$ the minimal $x \in X$ with $f(x) \neq 0$, where we assume, to guarantee the existence of such $x_{f}^{\min }$, that the function $f$ has a finite support $S=S_{f} \subset X$, or at least $S_{f}$ is bounded from below, i.e., $S_{f}$ contains at most finitely many elements which are $\preceq x_{0}$ for every $x_{0} \in X$. We also agree that $x_{f=0}^{\min }$ equals the empty set.

Given a linear space $D$ of functions on $X$ with the supports uniformly bounded from below, assign to it the union of the points $x_{f}^{\min }$ for all $f \in D$, denoted

$$
D \mapsto Y_{D}^{\min }=\bigcup_{f \in D}\left\{x_{f}^{\min }\right\} \subset X,
$$

and observe that

the cardinality of $Y_{D}^{\min }$ equals the rank of $D$,

$$
\left|Y_{D}^{\min }\right|=|D| .
$$

Example. If $X=\{1,2, \ldots, n\}$ then $Y_{D}^{\min }$ equals the set of the non-zero diagonal entries in a triangular matrix with $n$ columns and $|D|$ rows, where the rows make a basis in $D$.

\subsection{Domination by ordering and derivation of the linear algebraic isoperimetric} inequality from the combinatorial one. We start investigating

Isoperimetric Domination. Say that the isoperimetry of a linear space $L$ acted upon by a group $\Gamma$ dominates the isoperimetry of some set $X$ (non-linearly) acted by $\Gamma$ if there exits a $\Gamma$-equivariant (domination) map from the set $\mathscr{E}^{*}$ of finite dimensional subspaces $D \subset L$ to the set $2^{X}$ of subsets $Y \subset X$, say $D \mapsto Y_{D}$, such that $\left|Y_{D}\right|=|D|$ for all $D$ and

$$
\left|\operatorname{span}\left(D_{i}\right)\right| \geq\left|\cup_{i} Y_{D_{i}}\right| \quad(* \succ 0)
$$

for all finite collections of subspaces $D_{i}$.

Clearly,

the map $D \mapsto Y_{D}^{\min }$ based on a $\Gamma$-invariant order in $X$ is an isoperimetric domination.

It is also clear that

the existence of an isoperimetric domination makes $I_{*}(r ; L, \Gamma) \geq I_{\circ}(r ; X, \Gamma)$.

This leads to the following 
Equivalence $\circ \Longleftrightarrow *$ with order. Let an action of $\Gamma$ on $X$ preserve some order on $X$. Then the linear algebraic isoperimetric profile $I_{*}$ of (the space of functions with finite supports on) $X$ with respect to an arbitrary subset $G \subset \Gamma$ majorizes the combinatorial profile $I_{\circ}$ of $X$, and since, obviously, $I_{*} \leq I_{\circ}$, the two profiles are equal,

$$
I_{*}(r ; X, \Gamma)=I_{\circ}(r ; X, \Gamma) . \quad(\circ \Longleftrightarrow *)
$$

Furthermore, the $P$-functions of $X$ with respect to (the family of the orbit partitions of) arbitrary groups $\Gamma_{i}$ acting on $X$ satisfy

$$
P_{*}\left(n_{i}\right)=P_{\circ}\left(n_{i}\right) .
$$

Remark. Every domination map $\mathcal{E}^{*} \rightarrow 2^{X}$ is distance decreasing for the $\Gamma$-invariant metrics in $\mathscr{G}^{*}$ and in $2^{X}$ defined in 1.1, 1.4 and 1.5; this serves as good as the inequality $\left|\operatorname{span}\left(D_{i}\right)\right| \geq\left|\bigcup_{i} Y_{D_{i}}\right|$ if we work with the $I^{\max }$-profiles (compare $l_{p}$-non amenability in 2.2), while the profiles defined with the joint boundary (see 1.1) need more of the (lattice) structure in the spaces $\mathscr{E}^{*}$ and in $2^{X}$.

\subsection{Examples of orderable groups and corollaries to the equivalence $\circ \Longleftrightarrow *$.} Left orderable groups constitute an ample class of groups starting with $\Gamma=\mathbb{Z}$. They are built with the following constructions.

(1) Extensions. An extension $\Gamma$ of a left orderable group $\Gamma_{0}$ by a left orderable $\Gamma_{1}$ is left orderable by the lexicographic ordering construction. In particular

torsion-free polycyclic groups are orderable

and thus their isoperimetry extends to their group algebras.

(2) Limits and subgroups. Inductive and projective limits of orderable groups are, obviously, orderable and subgroups of orderable groups are orderable. It follows that

if every non-trivial finitely generated subgroup in $\Gamma$ admits a surjective homomorphism to $\mathbb{Z}$ then $\Gamma$ is left orderable.

In particular, the following groups are orderable,

(a) free groups $F_{k}$,

(b) wreath products $\Gamma$ of orderable groups,

(c) $\mathbb{Z}$-extension of torsion-free locally nilpotent groups by $\mathbb{Z}$.

Thus the equivalence $\circ \Longleftrightarrow *$ with order gives us yet another proof of the Elek theorem on non-amenability of the group algebras of $F_{k}$ for $k \geq 2$, and shows together with 8.2 that

the group algebra over a given field of a finitely generated amenable group may have arbitrarily fast growing Følner function. 
(3) Finite groups, and hence groups with non-trivial torsion, admit no left invariant order. In fact, if a linear space $D$ consists of functions on $\Gamma$ that are constant on the cosets of a finite subgroup $\Gamma_{0} \subset \Gamma$ and $G \supset \Gamma_{0}$, then, obviously, $I_{*}(|D|)<I_{\circ}(|D|)$.

(4) Amenable groups are orderable if and only if all their non-trivial (i.e. $\neq\{$ id $\}$ ) infinite subgroups admit epimorphisms to $\mathbb{Z}$ [22]. (The "if" part mentioned earlier is valid for all groups and is rather obvious.)

Thus, many virtually polycyclic (e.g. virtually free Abelian) groups are not orderable; Yet,

if $\Gamma$ is commensurable to a left orderable group, then $I_{*}$ is equivalent to $I_{\circ}$ for $\Gamma$.

In fact, if $\Gamma_{1} \subset \Gamma$ has finite index, or if $\Gamma$ admits an epimorphism on $\Gamma_{1}$ with a finite kernel, then the isoperimetric profiles of the two groups as well as of their group algebras are equivalent.

(5) The group homeo+ $(\mathbb{R})$ of orientation preserving homeomorphisms of the real line is left orderable and every countable left orderable group embeds into $h \operatorname{meo}_{+}(\mathbb{R})$. (This is well known and easy.) For example, the universal covering of $\mathrm{SL}_{2}(\mathbb{R})$ is left orderable and also

the ( finitely generated) Thompson group $T$ (dyadic homeomorphisms of an interval) is left orderable; thus, the (yet unknown) profile $I_{\circ}$ of $T$ is equal to $I_{*}$ of $T$.

3.4. $I_{*}$-profiles of groups with orderable subgroups. Let a finite subset $G_{0} \subset \Gamma$ generate an orderable group $\Gamma_{0} \subset \Gamma$, where $G_{0} \subset G^{\cdot m}$ for a finite generating subset $G \subset \Gamma$. Then the inequality $G^{\cdot m}$ from 1.4 combined with the equivalence $\circ \Longleftrightarrow *$ shows that

$$
I_{*}^{\max }(r ; \Gamma, G) \geq \frac{1}{m} I_{\circ}^{\max }\left(r ; \Gamma_{0}, G_{0}\right) .
$$

Examples. (a) Observe that the relative growth of $\Gamma_{0} \subset \Gamma$, denoted $G_{\circ \circ}(n)=$ $\left|G^{\cdot n} \cap \Gamma_{)}\right|$may be faster than the growth of $\Gamma_{0}$ with respect to any finite generating subset in $\Gamma_{0}$ if $\Gamma_{0}$ is "sufficiently distorted" in $\Gamma$. Then the following inequality, which can be derived from the above along with the displacement inequality (see 1.4), may serve better than just $F_{*}(n, \Gamma) \geq F_{*}\left(n, \Gamma_{0}\right)$,

$$
F_{*}^{\max }(n ; \Gamma, G) \geq \frac{1}{2} G_{\circ \circ}\left(\frac{n}{2}\right) .
$$

However, I see no example, with a finitely generated $\Gamma_{0}$, where one cannot do better by other means.

(b) If $\Gamma_{0}$ is infinitely generated, e.g, being the restricted Cartesian power of some finitely generated group $\Gamma_{1}$, then one can use measures $\mu$ on $\Gamma_{0}$ spread over large sets of "independent" elements in $\Gamma_{0}$. Such situation arises, for instance, for the wreath products $\Gamma$ of orderable finitely generated groups $\Gamma_{1}$ with finitely generated 
(not necessarily orderable) $\Gamma_{2}$, where one can apply the Loomis-Whitney inequality to obtain the lower bound

$$
F_{*}(n ; \Gamma) \succ \exp \left(G_{\circ}\left(n ; \Gamma_{2}\right) .\right.
$$

But, in truth, this holds for all (not necessarily orderable) groups $\Gamma_{1}$ (see 7.2) and if $\Gamma_{1}$ is not pure torsion, such an inequality is also valid for real $l_{2}$-functions and for decaying functions on $\Gamma$ with values in ultra metric fields (see 6.2).

3.5. $I_{*}$-profiles of groups with orderable factor groups. Consider an exact sequence

$$
1 \rightarrow \Gamma_{1} \rightarrow \Gamma \rightarrow \Gamma_{2} \rightarrow 1
$$

and let $G_{i} \subset \Gamma_{i}, i=1,2$, be finite symmetric generating subsets in $\Gamma_{i}$ containing the identity elements, where $G_{2}$ also denotes some lift of $G_{2}$ to $\Gamma$.

Orderable extension inequalities. Let $\Gamma_{2}$ be a left orderable group. Then the isoperimetric profile of the group algebra $A$ of $\Gamma$ is bounded from below by that of $\Gamma_{2}$,

$$
I_{*}(r ; \Gamma, G) \geq I_{*}\left(r ; \Gamma_{2}, G_{2}\right)=I_{\circ}\left(r ; \Gamma_{2}, G_{2}\right),
$$

for $G=G_{1} \cup G_{2} \subset \Gamma$.

Proof. Denote by $h$ the homomorphism $\Gamma \rightarrow \Gamma_{2}$, let $A_{\preceq \gamma_{2}} \subset A$, where $\gamma_{2} \in \Gamma_{2}$, consist of the functions $a$ on $\Gamma$ such that all $\gamma$ in the support $\operatorname{supp}(a) \subset \Gamma$ satisfy $h(\gamma) \preceq \gamma_{2}$ for a given left order on $\Gamma_{2}$ and define $A_{<\gamma_{2}} \subset A_{\leq \gamma_{2}}$ with the strict inequality $h(\gamma) \prec \gamma_{2}$.

Given a linear subspace $D \subset A$, let

$$
r=r\left(\gamma_{2}\right):=\operatorname{rank}\left(D \cap A_{\leq \gamma_{2}}\right) /\left(D \cap A_{\prec \gamma_{2}}\right)
$$

and observe that the $G_{2}$-boundary of the function $r$ on $\Gamma_{2}$ (i.e., the boundary of the subgraph of $r$ in $\Gamma_{2} \times \mathbb{Z}_{+}$) is bounded by the $G_{2}$-boundary of $D$,

$$
\left|\partial_{G_{2}}(r)\right| \leq\left|\partial_{G_{2}}(D)\right|
$$

and the bound on $I_{*}(\Gamma)$ by $I_{\circ}\left(\Gamma_{2}\right)$ follows as in 3.2.

Remark. We shall isolate in 5.11 a class of $\bullet$-convex functions $F$ and prove that

$$
\left.F_{*}\left(n_{1}, n_{2} ; \Gamma, G_{1}, G_{2}\right)\right) \geq F_{*}\left(n_{1} ; \Gamma_{i}, G_{1}\right) \cdot F_{\circ}\left(n_{2} ; \Gamma_{2}, G_{2}\right),
$$

when the functions $F_{*}\left(n_{1} ; \Gamma_{i}, G_{1}\right) \cdot F_{\circ}\left(n_{2} ; \Gamma_{2}, G_{2}\right)$ are $\bullet$-convex. 
3.6. Proof of Bartholdi's $I_{*}$-linearity theorem via orderings. The set $\mathcal{O}(X)$ of all orders $O$ on a countable set $X$ equals the projective limit of the orders on the finite subsets $Y$ in $X$; thus, it carries the structure of a compact topological space. The natural action of the group $\Gamma$ of all bijective transformations of $X$ is continuous on $\mathcal{O}(X)$, where it is free and transitive for finite $X$. (If $X$ is a finite set, then $\mathcal{O}(X)$ equals the set of the top dimensional simplices in the first barycentric subdivision of the regular $n$-simplex for $n=|X|-1$.) It is non-transitive for infinite $X$ as there are non-isomorphic orders and it is not free: the isotropy subgroup of a point $O$ in $\mathcal{O}(X)$ consists of the transformations preserving the order $O$.

There is a unique $\Gamma$-invariant probability measure, say $d O$ on $\mathcal{O}(X)$, which is the projective limit of the uniform measures on $\mathcal{O}(Y)$ for the finite subsets $Y \subset X$.

Given an order $O$ we invoke the (triangular basis) map from the set of all finite dimensional linear spaces of $\mathbb{F}$-functions on $X$ to the set of finite subsets in $X$,

$$
D \mapsto Y=Y_{D}^{\min }=Y_{D}^{\min }(O),
$$

associated to this $O$, and, following [2], let $\mu_{Y}=\mu_{D}^{O}$ be the measure on $X$ which assigns the unit weights to all points in $Y \subset X$ and which is zero outside $Y$. Clearly,

and

$$
\left|\mu_{D}^{O}\right|=|D|
$$

$$
D_{1} \subset D_{2} \Longrightarrow \mu_{D_{1}}^{O}(x) \leq \mu_{D_{2}}^{O}(x)
$$

for all $x \in X$.

Then we take the average over all orders $O$,

$$
\mu_{D}:=\int_{\mathcal{O}} \mu_{D}^{O} d O
$$

and observe that the measure $\mu_{D}$ on $X$, being an average, inherits the relations $(=)$ and $(\leq)$ from $\mu_{D}^{O}$; furthermore, since the measure $d O$ is $\Gamma$-invariant the map $D \mapsto \mu_{D}$ (unlike $D \mapsto \mu_{D}^{O}$ for a fixed order $O$ ) is $\Gamma$-equivariant.

The relations (=) and ( $\leq$ ) immediately imply (compare [2]) that $\left\|\mu_{D_{1}}-\mu_{D_{2}}\right\|_{l_{1}}=$ $\left|D_{1}\right|-\left|D_{2}\right|$ whenever $D_{1} \subset D_{2}$, and hence the map $D \mapsto \mu_{D}$ is 1-Lipschitz for the graph metric (see 1.11) in the space of $D$ 's (this is equivalent to the metric defined in 1.6) and the $l_{1}$-metric in the space of measures; then the implication non-amenability $\Longrightarrow I_{*}$-linearity follows as in 2.2 .

Remarks. (a) The above argument is a minor modification/simplification of the original one in [2], where the author defines the set $M_{D} \subset l_{1}(X) \subset l_{2}(X)$ of the measures $\mu_{Y}$ on $X$ associated to the subsets $Y \subset X$ such that the restriction map $D \mapsto D \mid Y$ is isomorphic, and then averages with the measure $d s$ on $M_{D}$ which is induced by the (multivalued) Gauss spherical (support) map of $M_{D}$ to the unit sphere in $l_{2}(X)$. 
(b) The (compact topological) $\Gamma$-spaces of orders on countable groups $\Gamma$ seem to have interesting dynamics, where, in particular, the presence of "dynamically small" invariant subsets and/or measures (e.g. with vanishing Kolmogorov entropy) may yield "good isoperimetry" in the linear spaces of functions on $\Gamma$.

\section{Families of measurable partitions and their invariants}

A Borel measurable (not necessarily measure preserving) map $P:(X, \lambda) \rightarrow\left(X^{\prime}, \lambda^{\prime}\right)$ between Borel measure spaces gives rise to a partition of $X$ into slices that are the pullbacks of points in $X^{\prime}$. If the map is surjective, we ascribe the notation $P$ to this partition, identify $X^{\prime}$ with the quotient space $X / P$ and denote the slice of $P$ through $x \in X$ by $P(x) \subset X$, using the same notation for $P(x) \in X / P$ as well as $P^{-1}\left(x^{\prime}\right)$, $x^{\prime}=P(x) \in X / P$.

These slices carry (almost everywhere defined and a.e. unique) the Fubini measure, denoted $\lambda / \lambda^{\prime}$ and also $d P(x)$, such that

$$
\int_{X} f(x) d \lambda=\int_{X / P} d \lambda^{\prime} \int_{P(x)} f(x) d P(x)
$$

for all measurable functions $f$ on $X$.

Coordinate lines and planes partitions. Every Cartesian product space

$$
X=\cdots \times X_{i} \times \cdots, \quad i \in I,
$$

comes along with an $I$-family ("web") of partitions, each into the $i$-th "coordinate lines", that are the copies of $X_{i}$ passing through the points in $X$. (These are the true parallel coordinate lines for the Euclidean spaces $X=\mathbb{R}^{|I|}=\cdots \times \mathbb{R} \times \cdots$ with the coordinates indexed by $i \in I$.) Moreover, $X$ supports the "web" of $2^{I}$ partitions corresponding to the "planes" indexed by subsets $J \subset I$ and representing $\times_{i \in J} X_{i}$.

Induced partitions. If $Y \subset X$ is a measurable subset, then the intersections of $Y$ with slices of a partition $P$ of $X$ are called $P$-slices ("threads") of $Y$. Thus we obtain a partition $P \mid Y$ of $Y$, where we disregard the empty intersections (or rather those of measure zero).

Orbit partitions. If $X$ is acted upon by a group $\Gamma$ then it is partitioned into the orbits of the action, where this partition is measurable for (measurably) proper actions.

In most of our examples we deal with countable, also called discrete, spaces where all partitions are measurable. If such a space $X$ is unitary, i.e., all atoms (points) have unit weights, then every partition $P$ equals the orbit partition of the group of the transformations of $X$ preserving the slices of $P$.

Remark. The only significant invariant of a measurable partition is the distribution of the values of the function $\lambda(P(x))$ on $X$ (or, equivalently, of the function $\lambda\left(P^{-1}\left(x^{\prime}\right)\right.$ ) 
on $X^{\prime}=X / P$ ). In particular, all partitions of discrete unitary spaces into countably many countable slices are mutually isomorphic. On the contrary, "measurable webs" that are families of partitions are vastly different and, typically, very rigid.

Example. The partitions of the 3-dimensional Heisenberg group $H$ into the orbits of the three standard generating subgroups uniquely determine the Lie groups structure in $H$ (this is easy); probably the same remains true for all families of orbits partitions of groups except for the obvious "exceptionally soft" cases (such as $Z^{k}$ generated by $k$ copies of $\mathbb{Z}$ ).

In the following sections we introduce several invariants of families of partitions starting with the well known picture of the isoperimetry in the free Abelian groups serving as a motivating example.

\subsection{Four proofs of the isoperimetric inequality for $\mathbb{Z}^{k}$}

$\mathbb{Z}^{k}$-inequality. The max-Følner function of the free Abelian group $\mathbb{Z}^{k}$ with the standard $k$ generators equals $n^{k}$.

The non-trivial part of the claim, the inequality

$$
F_{\circ}^{\max }\left(n ; \mathbb{Z}^{k}\right) \geq n^{k},
$$

follows by induction with either (a), (b) or (c) below.

Lemma. Let a countable set $X$ be acted upon by groups $\Gamma_{1}$ and $\Gamma_{2}$ with given finite generating subsets and $\Gamma$ be the group generated by $\Gamma_{1}$ and $\Gamma_{2}$.

(a) If the $\Gamma_{1}$ - and $\Gamma_{2}$-orbits are transversal, i.e., intersect at single points, and if the $\Gamma_{2}$-orbits are infinite, or

(b) if the transformations from $\Gamma_{2}$ send $\Gamma_{1}$-orbit to $\Gamma_{1}$-orbits and the orbits of the resulting action of $\Gamma_{2}$ on the quotient space $X / \Gamma_{1}$ are all infinite, then

$$
F_{\circ}^{\max }(n ; X ; \Gamma) \geq n \cdot F_{\circ}^{\max }\left(n ; X ; \Gamma_{1}\right) .
$$

(c) If $\Gamma_{2}$ sends $\Gamma_{1}$-orbits to $\Gamma_{1}$-orbits as in (b) and all $\Gamma_{1}$-orbits are infinite, then

$$
F_{\circ}^{\max }(n ; X ; \Gamma) \geq n \cdot F_{\circ}^{\max }\left(n ; X / \Gamma_{1} ; \Gamma_{2}\right) .
$$

Proof. (a) Take a $Y \subset X$ and look at the two partitions of it where the slices are the intersections of $Y$ with the $\Gamma_{1}$ - and $\Gamma_{2}$-orbits. If

$$
\left|\partial_{\Gamma_{1}}(Y)\right| \leq \frac{1}{n_{1}}|Y|
$$


then there is a $\Gamma_{1}$-slice of $Y$, say $S_{1}$, such that

$$
\left|\partial_{\Gamma_{1}}\left(S_{1}\right)\right| \leq \frac{1}{n_{1}}\left|S_{1}\right|
$$

and by the definition of the Følner function for $\left(X, \Gamma_{1}\right)$,

$$
\left|S_{1}\right| \geq F_{\circ}^{\max }\left(n_{1} ; X, \Gamma_{1}\right) .
$$

Since the $\Gamma_{2}$-orbits are infinite, each non-empty $\Gamma_{2}$-slice of $Y$ contributes at least one point to the $\Gamma_{2}$-boundary, and the number of the $\Gamma_{2}$-slices that meet $S_{1}$ is $\geq\left|S_{1}\right|$ due to the transversality of the two partitions. If, furthermore,

$$
\left|\partial_{\Gamma_{2}}(Y)\right| \leq \frac{1}{n_{2}}|Y|
$$

then

$$
|Y| \geq n_{2} \cdot\left|\partial_{\Gamma_{2}}(Y)\right| \geq n_{2} \cdot\left|S_{1}\right| \geq n_{2} \cdot F_{*}^{\max }\left(n_{1} ; X ; \Gamma_{1}\right),
$$

and the required bound on $F_{\circ}^{\max }(n ; \Gamma)$ follows if we take $n_{1}=n_{2}=n$.

(b) Consider the subsets $\underline{Y}_{m} \subset X / \Gamma_{1}$ of those $\Gamma_{1}$-orbits that meet $Y$ at $\geq m$ points, where $m=1,2, \ldots,\left|S_{1}\right|$ for the above $S_{1}$. Observe that

$$
\left|\partial_{\Gamma_{2}}(Y)\right| \geq \sum_{m=1,2, \ldots,\left|S_{1}\right|}\left|\partial_{\Gamma_{2}}\left(\underline{Y}_{m}\right)\right| \geq\left|S_{1}\right|
$$

and conclude the proof as in (a).

(c) Since

$$
|Y|=\sum_{m}\left|\underline{Y}_{m}\right|
$$

and

$$
\left|\partial_{\Gamma_{2}}(Y)\right| \geq \sum_{m}\left|\partial_{\Gamma_{2}}\left(\underline{Y}_{m}\right)\right|
$$

the cardinality of $\underline{Y}_{1}$ satisfies

$$
\left|\underline{Y}_{1}\right|=\max _{m}\left|\underline{Y}_{m}\right| \geq F_{\circ}\left(n_{2} ; X / \Gamma_{2}, \Gamma_{2}\right) .
$$

Since each $\Gamma_{1}$-slice of $Y$ contributes a point to the $\Gamma_{1}$-boundary of $Y$, the subset $\underline{Y}_{1} \subset X / \Gamma_{1}$, which is the set of the $\Gamma_{1}$-slices of $Y$, is bounded by

$$
\left|\underline{Y}_{1}\right| \leq\left|\partial \Gamma_{1}(Y)\right| \leq \frac{1}{n_{1}}|Y|,
$$

and the claim follows. 
Multivariable Følner function of $\mathbb{Z}^{k}$. Recall that the multivariable Følner function $F_{\circ}\left(n_{i} ; X, G_{i}\right)$ for several sets of transformations $G_{i}$ acting on $X$ is defined as the maximal $N$ such that for every $Y \subset X$ with

$$
\left|\partial_{G_{i}}(Y)\right| \leq \frac{1}{n_{i}}|Y|
$$

it holds that

$$
|Y| \geq N
$$

Multivariable isoperimetric inequality for $\mathbb{Z}^{k}$. The following holds:

$$
F_{\circ}\left(n_{1}, \ldots, n_{k} ; \mathbb{Z}^{k}, g_{1}, \ldots, g_{k}\right)=n_{1} \cdot \ldots \cdot n_{k}
$$

for every set of generators $g_{1}, \ldots, g_{k}$ in $\mathbb{Z}^{k}$.

Proof. Since the orbits $S$ of all $k$ subgroups $\Gamma_{i}=\mathbb{Z}$ in $\mathbb{Z}^{k}$ generated by $g_{i}$ are infinite, every $S$ that meets a finite set $Y \subset \mathbb{Z}^{k}$ contributes at least one point to its boundary; thus, $\left|\partial_{g_{i}}(Y)\right|$ is bounded from below by the number $m_{i}$ of the $\Gamma_{i}$-orbits $S$ that meet $Y$.

These numbers satisfy $\prod_{i} m_{i} \geq|Y|^{k-1}$ by the Loomis-Whitney inequality (see 1.10); hence $F_{\circ} \geq \prod n_{i}$, while the opposite inequality is obvious.

About $F_{*}$. It is unclear how much of (a), (b) and (c) remain valid for linear actions. Yet, we shall see in 6.2 that (b) holds for $\Gamma$ acting on the space of functions on $X$ with finite supports if, for example, there is an element of infinite order in $\Gamma_{2}$ freely acting on $X / \Gamma_{1}$. Also we prove in 7.2 a (coarse entropic) version of (b) for $F_{*}$ in the general case.

4.2. Følner transform $\boldsymbol{F}_{\odot}$ and Schwartz symmetrization. Given a family $\mathcal{P}$ of measurable partitions $P_{i}$, define the Folner transform (composition) $F_{\odot}:\left(F_{i}\right) \mapsto$ $F=F\left(n_{i}\right)$, where $F_{i}=F_{i}(n), n \in[0, \infty)$, are monotone increasing positive functions, as follows. Take $I_{i}=I_{F_{i}}$ for $I_{F_{i}}(r)=r J_{i}(r)$ where $J_{i}(r)$ stands for the inverse function of $F_{i}(n)$ (i.e., $F=F_{I}$, for this $I$ compare 1.9), take the integral over $Y / P_{i}$ of the values of $I_{i}$ at the Fubini measures of all $P_{i}$-slices $S$ of $Y$ and denote

$$
|\partial|_{I_{i}}(Y)=\int_{Y / P_{i}} I_{i}(|S|) .
$$

Finally, let $F\left(n_{i}\right)=F_{\odot}\left(F_{i}\left(n_{i}\right)\right)$ be the maximal $N$ such that every $Y$ with $|\partial|_{I_{i}}(Y) \leq$ $\frac{1}{n_{i}}|Y|$ has $|Y| \geq N$.

Example. If a (discrete or locally compact) group $\Gamma$ is partitioned into the orbits of (closed ) subgroups $\Gamma_{i} \subset \Gamma$, then the multivariable $F \varnothing l$ ner function of $\Gamma$ with respect 
to given generating subsets $G_{i} \subset \Gamma_{i}$, obviously, satisfies (with the obvious extension of the notations to the locally compact non-discrete case)

$$
F_{\circ}\left(n_{i} ; \Gamma, G_{i}\right) \geq F_{\odot}\left(F_{i}\left(n_{i} ; \Gamma_{i}, G_{i}\right)\right) .
$$

$P_{\circ}$-functions and the Følner transform. The $P_{\circ}$-function defined in 1.10 clearly equals the Følner transform of the functions $F\left(n_{i}\right)=n_{i}$.

Schwartz symmetrization. If $X=X_{1} \times X_{2}$ with the two coordinate partitions then the corresponding Følner transform (composition) is denoted

$$
F_{1} \cdot F_{2}:=F_{\odot}\left(F_{1}, F_{2} ; X_{1} \times X_{2}\right) .
$$

It is obvious that $F_{1} \bullet F_{2} \leq F_{1} \cdot F_{2}$.

Let us model the general product space by $X=\mathbb{R}_{+}^{2}$, where we consider closed monotone subsets $Y \subset X$, which means that the intersections of $Y$ with the vertical lines $x_{1} \times \mathbb{R}_{+}$are segments $x_{1} \times\left[0, x_{2}=f_{1}\left(x_{1}\right)\right]$ and the intersections with the horizontal ones $\mathbb{R}_{+} \times x_{2}$, are segments $\left[0, x_{1}=f_{2}\left(x_{1}\right)\right] \times x_{2}$, where $f_{1}$ and $f_{2}$ are mutually inverse monotone decreasing functions with the common graph serving as the boundary of $Y$.

Let $I_{i}(r)=I_{F_{i}}(r)$, for $i=1,2$ and $0 \leq r<\infty$, be "the isoperimetric profiles" corresponding to $F_{i}$, set

$$
\mathcal{I}_{i}(Y)=\int_{0}^{\infty} I_{i}\left(f_{i}(r)\right) d r
$$

and define $F\left(n_{1}, n_{2}\right)$ as the infimum of the measures $\mu$ of monotone subsets $Y \subset \mathbb{R}_{+}^{2}$ that satisfy

$$
\mathcal{I}_{i}(Y) / \mu(Y) \leq n_{i}
$$

It is easy to see (we shall not use it in sequel) that

if $I_{i}$ are positive concave monotone increasing functions, then this $F$ equals the Følner transform of the functions $F_{i}$ corresponding to $I_{i}$,

$$
F=F_{1} \bullet F_{2} \text {. }
$$

For example, if the extremal $Y=Y\left(n_{1}, n_{2}\right)$ are rectangular for all $n_{1}, n_{2} \geq 0$, (i.e., $\left.Y=\left[0, x_{1}\right] \times\left[0, x_{2}\right] \subset \mathbb{R}_{+}\right)$then the Følner functions $F_{i}$ corresponding to $I_{i}$ satisfy $F_{1} \bullet F_{2}=F_{1} \cdot F_{2}$.

In general, the extremal $Y$ are bounded by broken lines consisting of finitely many vertical and horizontal segments under mild regularity/genericity assumptions on $I_{i}$, and thus the problem of evaluation $F=F_{1} \cdot F_{2}=F_{1} \cdot F_{2}$ reduces to a finite dimensional variational problem.

Similar considerations also apply to the Cartesian products of more than two spaces. But this does yield any explicit formula for the Følner transform, nor does it deliver a transparent criterion for the equality $F_{1} \bullet F_{2}=F_{1} \cdot F_{2}$. 
On the brighter side, we shall prove in 5.11 this equality under the assumption of convexity of $\log F\left(\frac{1}{\varepsilon}\right)$.

Schwartz Symmetrization for group extensions. Let $\Gamma$ be an extension of $\Gamma_{1}$ by $\Gamma_{2}$,

$$
1 \longrightarrow \Gamma_{1} \longrightarrow \Gamma \longrightarrow \Gamma_{2} \longrightarrow 1,
$$

let $G_{i} \subset \Gamma_{i}, i=1,2$, be generating subsets and let us, to save notation, use $G_{2}$ for some lift of $G_{2}$ to $\Gamma$. Then the maximal Folner Functions of $\Gamma$ for $G_{1}$ and every lift $G_{2}^{\prime} \subset \Gamma$ of $G_{2}$ to $\Gamma$ are bounded from below by the Folner functions $F_{i}$ of $\Gamma_{i}, i=1,2$ in the same way as the Folner function of the Cartesian product $\Gamma_{1} \times \Gamma_{2}$ is,

$$
F_{\circ}(\Gamma) \geq F_{1} \cdot F_{2} .
$$

Proof. Given a subset $Y \subset \Gamma$ denote by $m(\gamma), \gamma \in \Gamma_{2}$, the number of points in $Y$ which go to $\gamma$ under the homomorphism $\Gamma \rightarrow \Gamma_{2}$ and consider the subgraph $Y_{S} \subset \Gamma_{2} \times \mathbb{Z}_{+}$of the function $m(\gamma)$, that is the set of pairs $(\gamma \in \Gamma, i=1,2, \ldots$, such that $m(\gamma) \leq i$. Then the proof follows by observing that $\left|\partial_{G_{2}}\left(Y_{S}\right)\right| \leq\left|\partial_{G_{2}^{\prime}}(Y)\right|$ for the obvious action of $\Gamma_{2}$ on $\Gamma_{2} \times \mathbb{Z}_{+}$.

Remark. In order to apply this, we need a computable lower bound on the --product. A rough such bound follows with the $F_{\odot} \succ E_{\circ}$ inequality in 4.5 and the sharp one is established in 5.11 for the entropic Følner functions $F_{\bullet}(n)$, where $\log F_{\bullet}\left(\frac{1}{\varepsilon}\right)$ is, by definition, convex.

Question. Suppose that the ratio

$$
I_{\circ}\left(r ; \Gamma, G_{1}, G_{2}\right) / I_{\circ}\left(r ; \Gamma_{1} \times \Gamma_{2}, G_{1}, G_{2}\right)
$$

remains bounded as $r \rightarrow \infty$. Is then $\Gamma$ commensurable with $\Gamma_{1} \times \Gamma_{2}$, provided that the groups are amenable?

(Also the random walk on a non-trivial extension of groups, probably, dissipates faster than that on the products as it happens to the Brownian motion on vector bundles according to the Kac-Feynman formula that would be, for group extensions, a comparison relation between the $G_{1}$-random walk on $\Gamma_{1}$ with the dissipation rate of the measures on $\Gamma_{1} \subset \Gamma$ coming from the paths in $\Gamma \supset \Gamma_{1}$ that return to id down in $\Gamma_{2}$.)

4.3. $\boldsymbol{E}_{\mathrm{o}}$-functions and $\boldsymbol{E}_{\mathrm{o}}$-inequalities. We have the following

Definition of $E_{\mathrm{o}}$. Given a measure space $X$ with a countable family of partitions $P_{i}, i=I$, define the (expansion) $E_{\circ}$-function $E_{\circ}\left(L_{i}\right)=E_{\circ}\left(L_{i} ; P_{i}\right)$ for $L_{i} \in[0, \infty]$ as the supremum of the functions $E\left(L_{i}\right)$ such that every subset $Y \subset X$, all of whose 
(non-empty!) $P_{i}$-slices have their (Fubini) measures $\geq L_{i}$ for all $i \in I$, satisfies the following lower bound on its own measure:

$$
|Y| \geq E_{\circ}\left(L_{i}\right) .
$$

(Compare Lemma 1 in [9].)

Example ( $E_{\circ}$ for cartesian products). Let $X$ be the Cartesian product $X=\times_{i \in I} X_{i}$ with the product measure, and let $P_{i}$ be the coordinate "line" partitions with the respective slices isomorphic to (the copies of) $X_{i}$ in $X$. Then, obviously,

$$
E_{\circ}\left(L_{i}\right)=\prod_{i \in I} L_{i}
$$

Let us generalize this to the coordinate "plane" partitions with the slices (isomorphic to the copies of ) $\times_{i \in J} X_{i}$ for (all) subsets $J \in I$.

Define a partition of unity on $I$ as an assignment of a non-negative number $\alpha_{J}$ to each $J \subset I$ such that $\sum \alpha_{J} \chi_{J}=1$, where $\chi_{J}: I \rightarrow\{0,1\} \subset \mathbb{R}$ denote the characteristic (indicator) functions of the subsets $J \subset I$.

Coordinate $\boldsymbol{E}_{\mathrm{o}}$-Inequalities. The $E_{\mathrm{o}}$-function of the family of the partitions $P_{J}$ is bounded from below by,

$$
E_{\circ}\left(L_{J}\right) \geq \prod_{J \subset I} L_{J}^{\alpha_{J}}
$$

for all partitions of unity of $I$.

Proof. The equality $(E \Pi)$ shows that the minima $l(J)$ of the logs of the measures of $P_{J}$-slices of $Y$ are sub-additive,

$$
l\left(J_{1}\right)+l\left(J_{2}\right) \leq l\left(J_{1} \cup J_{2}\right),
$$

for all pairs of disjoint subsets $J_{1}, J_{2} \subset I$. Then $\left(E \Pi^{\alpha}\right)$ follows by the inclusionexclusion principle for sub-additive set functions.

Questions. Can one evaluate $E_{\circ}$ for standard groups $(\Gamma, G)$ with families of cyclic subgroups $C_{g} \subset \Gamma$ generated by $g \in G$ ? For example, what is $E_{\circ}\left(\Gamma, C_{g}\right)$ for the free nilpotent and solvable group $\Gamma$ on a given generating set $G$ with a prescribed nilpotency (solvability) degree? Does the function $E_{\circ}$ change much if one augments the collection $C_{g}$ with extra (cyclic) subgroups?

Let $\mathbb{F}$ be a finite field, $\Gamma$ be the special linear group and let $\Gamma_{i}$ be some "standard" subgroups, e.g., the $k$ subgroups $\mathrm{SL}_{k-1}(\mathbb{F})$ positioned inside $\mathrm{SL}_{k}(\mathbb{F})$ in the $k$ obvious ways.

What is the expansion function for these? Can one prove the isoperimetric inequality for $\Gamma=\mathrm{SL}_{k}(\mathbb{F})$ by some slicing argument? (This would bring us closer to the $T$-property for $\mathrm{SL}_{k}(\mathbb{R})$.) 
Can one meaningfully bound from below the cardinality of $G^{\cdot m} \subset \mathrm{SL}_{k}(\mathbb{F})$, say for $m=2$, for subsets $G \subset \mathrm{SL}_{k}(\mathbb{F})$ with $|G| \geq \varepsilon \cdot\left|\mathrm{SL}_{k}(\mathbb{F})\right|$, where $\varepsilon$ is somewhat greater than $\frac{1}{2 k}$ ?

\subsection{Slice removal and slice decomposition}

Decomposition lemma. Let $Y \subset X$ have the measure $<E_{\circ}\left(L_{i}\right), i \in I$, for given partitions $P_{i}$ of $X$ and numbers $L_{i}$. Then $Y$ can be decomposed into disjoint measurable subsets $Y_{i} \subset Y$ such that all $P_{i}$-slices of $Y_{i}$ have measures $\leq L_{i}$ for all $i \in I$.

Proof. Keep removing slices $S_{i}$ from $Y$ of measures $\leq L_{i}$.

Corollary (Slicing inequality for $\boldsymbol{F}_{\Sigma}^{\text {incr }}$ ). Let $F_{i}(n)$ and $I_{i}(r)$ be some "Følner" functions and the corresponding "profiles", and let the "boundary" of an $Y \subset X$ be understood as the sum of the $I_{i}$-"boundaries"

$$
|\partial|_{\Sigma}(Y)=\sum_{i}|\partial|_{I_{I}}(Y)
$$

If the functions $I_{i}(r)$ are monotone increasing, then the "Folner function" of $\left(X, P_{i}\right)$ associated to $|\partial|_{\Sigma}$ (a kind of a diagonal Folner transform of $F_{i}$ ) satisfies

$$
F_{\Sigma}\left(n ; X, P_{i}\right) \geq F_{\text {incr }}(n) \geq E_{\circ}\left(L_{i}=F_{i}(n)\right),
$$

where $F_{\mathrm{incr}}(n)$ is a function for which the corresponding $I(r)$ is monotone increasing.

Proof. Decompose a given $Y \subset X$ into the union of $Y_{i}$ and observe that $|\partial|_{I_{i}}\left(Y_{i}\right) \leq$ $|\partial|_{I_{i}}(Y)$ by the monotonicity of $I_{i}$. Adding these inequalities and recalling the definitions of $F_{i}$ and $I_{i}$ we obtain the required lower bound on $|\partial|_{\Sigma}(Y)$.

Remark. This inequality is not sharp. It can be slightly improved by taking into account by how much the measure of $Y$ diminishes as we remove subslices from it; but this makes the inequality only marginally better.

Group-subgroups example. Let $\Gamma$ be a group and $\Gamma_{i}$ be subgroups in $\Gamma$ with generating subsets $G_{i} \subset \Gamma_{i}$, and let $I_{i}(r)$ be some monotone minorants of the isoperimetric profiles $I_{\circ}\left(r ; \Gamma_{i}, G_{i}\right)$. Then there is a monotone function $I(r)$ minorizing the isoperimetric profile of $\Gamma$ defined with $|\partial|_{\Sigma}=\sum_{i}|\partial|_{G_{i}}$ such that the Følner function associated to this $I$ is bounded from below by $E_{\circ}\left(L_{i}=F_{i}(n)\right)$ for $F_{i}$ associated to $I_{i}$ and with $E_{\circ}$ referring to the partitions of $\Gamma$ ) into the $\Gamma_{i}$-orbits.

$\mathbb{Z}^{k}$-subexample. The above applies to the free Abelian group $\mathbb{Z}^{k}$ with the standard generators $g_{1}, g_{2}, \ldots, g_{k}$ and the corresponding "coordinate lines" partitions into the 
orbits of the cyclic subgroups generated by $g_{i}$; but the resulting inequality is only asymptotically sharp.

Similarly, every Cartesian product space $X=\left(X, \mathrm{X}_{i} \Gamma_{i}\right)=\mathrm{X}_{i}\left(X_{i}, \Gamma_{i}\right)$ satisfies

$$
F_{\circ}^{\mathrm{inc}, \Sigma}(n ; X) \geq \prod_{i} F_{\circ}^{\mathrm{incr}}\left(n ; X, \Gamma_{i}\right) .
$$

4.5. Lower bounds on $\boldsymbol{F}_{\odot}$ by $\boldsymbol{E}_{\text {o }}$. Given a partition $P$ of a measure space $X$ and a subset $Y \subset X$, denote by sup $|Y \cap P|$ the supremum of the Fubini measures of the slices of $P$ intersected with $Y$ and by $\inf |Y \cap P|$ the infimum of these measures taken over the slices that have strictly positive intersection measures with $Y$.

Then, for each number $\kappa>0$, consider subsets $Y^{\prime}=Y^{\prime}(\kappa) \subset Y$ such that sup $\left|Y^{\prime} \cap P\right|<\kappa$ and denote by $\left|P^{<\kappa}(Y)\right|$ the supremum of the measures of these $Y^{\prime}$.

One can bound $\left|P^{<\kappa}(Y)\right|$ in terms of the distribution of the (Fubini) measures of the $P$-slices of $Y$ as follows:

If the union $Y_{<\lambda} \subset Y$ of the slices of measures $<\lambda$ satisfies $\left|Y_{<\lambda}\right| \leq \varepsilon|Y|$ for some $\lambda \geq \kappa$ and $\varepsilon \geq 0$, then

$$
\left|P^{<\kappa}(Y)\right| \leq \varepsilon|Y|+c(1-\varepsilon)|Y| \text { for } c=\kappa / \lambda .
$$

In fact, the intersection of every $Y^{\prime}=Y^{\prime}(\kappa)$ with $Y_{<\lambda}$ has measure $\leq \varepsilon_{\lambda}|Y|$ for $\varepsilon_{\lambda}=\left|Y_{<\lambda}\right| /|Y|$, while the intersection of $Y^{\prime}$ with the complement $Y_{\geq \lambda}=Y \backslash Y_{<\lambda}$ has measure $\leq c\left(1-\varepsilon_{\lambda}\right)|Y|$. This implies $[c \lambda \kappa]$ with $\varepsilon_{\lambda}$ instead of $\varepsilon$ and, hence, with $\varepsilon$ itself, since $\varepsilon \leq \varepsilon_{\lambda}$ and $c \leq 1$.

Slice removal lemma (Compare Lemma 1 in [9]). Let $P_{i}, i \in I$, be partitions of a measure space $X$, let $Y \subset X$ and let $\kappa_{i}$ be positive numbers such that

$$
\sum_{i \in I}\left|P_{i}^{<\kappa_{i}}(Y)\right|<|Y| .
$$

Then there exists a subset $Y_{\odot} \subset Y$ of positive measure such that

$$
\inf \left|Y_{\odot} \cap P_{i}\right| \geq \kappa_{i} \quad \text { for all } i \in I .
$$

Proof. Let $Y_{\odot}$ be a maximal subset in $Y$ with

$$
\inf \left|Y_{\odot} \cap P_{i}\right| \geq \kappa_{i} \quad \text { for all } i \in I,
$$

i.e., having all $P_{i}$-slices of measure $\geq \kappa_{i}$ for all $i$. (Such a maximal $Y_{\odot}$ is, in fact, unique, since the union of two subsets with $P_{i}$-slices $\geq \kappa_{i}$ also has the slices $\geq \kappa_{i}$.) Then every subset in the complement, say $Z \subset Y \backslash Y_{\odot}$, contains a point $x \in Z$ such that the slice $S=S(x, i)$ of some $P_{i}$ passing through $x$ has $|S \cap Z|<\kappa_{i}$. Therefore, 
$Y \backslash Y_{\circ}$ decomposes (as in 2.3 (B), p. 521) into the union of subsets $Z_{i}, i \in I$, where each $Z_{i}$ has $\sup \left|Z_{i} \cap P\right|<\kappa_{i}$, i.e., it has all $P_{i}$-slices of measures $<\kappa_{i}$. Thus

$$
\left|Y_{\odot}\right| \geq|Y|-\sum_{i \in I}\left|P_{i}^{<\kappa_{i}}(Y)\right|>0
$$

and the claim follows.

On the average measures of slices. Denote by $Y_{i}=Y\left(\kappa_{i}\right) \subset Y$ the union of the $P_{i}$-slices of $Y$ of measures $\geq \kappa_{i}$ and observe that $\cap_{i} Y_{i} \supset Y_{\odot}$; thus, the inequality $\sum_{i} P_{i}^{\kappa_{i}}(Y)<|Y|$ guarantees that the intersection $\cap_{i} Y_{i}$ is non-empty. In fact, one only needs for this the total bound on $\delta_{i}=1-\left|Y_{i}\right| /|Y|$, namely $\sum_{i} \delta_{i}<1$, but the latter does not provide any lower bound on the cardinalities of all slices of $\cap_{i} Y_{i}$. However, if the sum $\sum_{i} \delta_{i}$ is small, then the $P_{i}$-slices of $\cap_{i} Y_{i}$ are "large on the average" in the following sense.

Let $d x_{i}$ denote the measure on the quotient space $X_{i}=X / P_{i}$ associated to the partition $P_{i}$ of $X$ and let us regard the Fubini measures of the $P_{i}$-slices of subsets $Y \subset X$ as functions on $X_{i}$ denoted $\left|S\left(x_{i}\right)\right|(Y), x_{i} \in X_{i}$. Then $\int_{X_{i}}\left|S\left(x_{i}\right)\right|(Y) d x_{i}=$ $|Y|$ for all $Y \subset X$ by the definition of the Fubini measures and the average measure of the non-empty (or, rather, of positive measure) $P_{i}$-slices of $Y$ is (defined as)

$$
\left(\int_{X_{i}}\left|S\left(x_{i}\right)\right|(Y) d x_{i}\right) /\left|Y / P_{i}\right|=|Y| /\left|Y / P_{i}\right|
$$

Since the non-empty slices of every $Y_{i}$ have measures $\geq \kappa_{i}$,

$$
\left|Y_{i}\right| /\left|Y_{i} / P_{i}\right|=\left(\int_{X_{i}}\left|S\left(x_{i}\right)\right|\left(Y_{i}\right) d x_{i}\right) /\left|Y_{i} / P_{i}\right| \geq \kappa_{i}
$$

and the average measure of the non-empty $P_{i}$-slices of the intersection $\cap_{i} Y_{i}$ is bounded from below for all $i$ by

$$
\left.\left|\left(\bigcap_{i} Y_{i}\right)\right| /\left|\left(\bigcap_{i} Y_{i}\right) / P_{i}\right| \geq\left|\bigcap_{i} Y_{i}\right| /\left|Y / P_{i}\right| \geq \kappa_{i}\left(1-\sum_{i} \delta_{i}\right) . \quad\left(\left[\bigcap_{i} / P_{i}\right)\right]\right)
$$

Evaluation of $\boldsymbol{F}_{\odot}$ by $\boldsymbol{E}_{\circ}$ (Compare [9]). The Følner transform for every family of partitions $P_{i}$ is bounded from below by the $E_{\circ}$-function,

$$
F\left(n_{i}\right)=F_{\odot}\left(F_{i}\left(n_{i}\right) ; P_{i}\right) \geq E_{\circ}\left(c_{i} F_{i}\left(\varepsilon_{i} n_{i}\right)\right), \quad\left(F_{\odot} \succ E_{\circ}\right)
$$

where $0 \leq c_{i}, \varepsilon_{i} \leq 1$ are arbitrary numbers satisfying

$$
\sum_{i}\left(\varepsilon_{i}+c_{i}\left(1-\varepsilon_{i}\right)\right)<1
$$


Proof. If $|\partial|_{I_{i}}(Y) \leq|Y| / n_{i}$, then the measure of the subset $Y_{<\lambda_{i}} \subset Y$ that is the union of the $P_{i}$-slices of $Y$ with measures $<\lambda_{i}=F_{i}\left(\varepsilon_{i} n_{i}\right)$ satisfies $\left|Y_{<\lambda_{i}}\right| \leq \varepsilon_{i}|Y|$

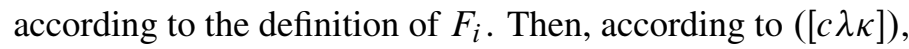

$$
\left|P_{i}^{<\kappa_{i}}(Y)\right| \leq\left(\varepsilon_{i}+c_{i}\left(1-\varepsilon_{i}\right)\right)|Y|
$$

and the slice removal lemma yields the required bound

$$
F\left(n_{i}\right) \geq|Y| \geq\left|Y_{\odot}\right| \geq E_{\circ}\left(\kappa_{i}=c_{i} F_{i}\left(\varepsilon_{i} n_{i}\right)\right) .
$$

Product example. The multivariable Folner function $F$ of the Cartesian product $X$ of $\Gamma$-spaces $X_{i}, i=1,2, \ldots, k$, is bounded by the Folner functions $F_{i}$ of $X_{i}$ by

$$
F\left(n_{i}\right) \geq \prod_{i} c_{i} F_{i}\left(\varepsilon_{i} n_{i}\right)
$$

provided that $\sum_{i}\left(\varepsilon_{i}+c_{i}\left(1-\varepsilon_{i}\right)\right) \leq 1$, e.g., for $\varepsilon_{i}=1 / 2 k$ and $c_{i}=1 /(2 k-1)$. For instance, if $k=2$, then

$$
F_{\circ}(n ; X) \geq \frac{1}{9} F_{\circ}\left(\frac{n}{4} ; X_{1}\right) \cdot F_{\circ}\left(\frac{n}{4} ; X_{2}\right) .
$$

(Sharper inequalities are available for •-convex $F_{i}$, see 5.11.)

4.6. $E_{\circ}$-functions and group extensions with and without distortion. Let $\Gamma_{1}$ be a normal subgroup in $\Gamma$ and $\Gamma_{2}=\Gamma / \Gamma_{1}$. Then the above inequality conjunction with the Schwartz symmetrization shows that

$$
F_{\circ}\left(n ; \Gamma, G_{1} \cup \widetilde{G}_{2}\right) \geq \frac{1}{9} F_{\circ}\left(\frac{n}{4} ; \Gamma_{1}\right) \cdot \frac{1}{4} F_{\circ}\left(\frac{n}{4} ; \Gamma_{2}, G_{2}\right)
$$

where $\widetilde{G}_{2} \subset \Gamma$ is some lift of $G_{2}$ to $\Gamma$. Furthermore, if $\Gamma_{2}$ is orderable, then

$$
F_{*}\left(n ; \Gamma, G_{1} \cup \widetilde{G}_{2}\right) \geq \frac{1}{9} F_{*}\left(\frac{n}{4} ; \Gamma_{1}\right) \cdot \frac{1}{4} F_{\circ}\left(\frac{n}{4} ; \Gamma_{2}, G_{2}\right) .
$$

Bound $\boldsymbol{F}_{\odot} \succ \boldsymbol{E}_{\circ}$ with distorted subgroups. The above lower bound $F_{\odot} \succ E_{\circ}$ on the Følner function of a group $\Gamma$ by those of given subgroups $\Gamma_{i} \subset \Gamma$ and the $E_{\mathrm{o}}$-functions of the family of the partitions of $\Gamma$ into $\Gamma_{i}$-orbits can be sometimes strengthened if (some of) the subgroups $\Gamma_{i}$ are "sufficiently distorted" in $\Gamma$.

Namely, let $\mu_{i}$ be probability measures supported on $\Gamma_{i} \cap G^{\cdot m_{i}}$ for a given finite generating subset $G \subset \Gamma$, and let $F_{\mu_{i}}$ be the Følner functions of $\Gamma_{i}$ associated to $\partial_{\mu_{i}}$. Then $F_{\odot} \succ E_{\circ}$ provides the lower bound on $F_{\circ}\left(n, \Gamma, \mu_{i}\right)$ in terms of $F_{\mu_{i}}$, where, in turn, the $G$-boundary is bounded from below by $\frac{1}{m_{i}} \cdot \partial_{\mu_{i}}$ for all $i$ according to the $G^{\cdot m}$-inequality from 1.4 . 
If one takes $\mu_{i}$ uniformly spread over $\Gamma_{i} \cap G^{\cdot m_{i}}$, then one can apply the displacement inequality from 1.4, p. 504. For instance, if the subgroups $\Gamma_{i}$ are infinite cyclic with the relative growth functions $G_{i}(n):=\left|\Gamma_{i} \cap G^{\cdot n}\right| \succ \succ n$, then one gains by substituting $L_{i} \mapsto G_{i}\left(L_{i}\right)$ in $E_{\mathrm{o}}$.

If one thus capitalizes on distortion of a normal subgroup $\Gamma_{1}$ in $\Gamma$ in $\left(\frac{1}{4} \circ\right)$ then one replaces $F_{\circ}\left(\frac{n}{4} ; \Gamma_{1}\right)$ in $\left(\frac{1}{4} \circ\right)$ by $\frac{1}{2} G_{\circ \circ}\left(\frac{n}{2}\right)=\frac{1}{2}\left|\Gamma_{1} \cap\left(G_{1} \cup \widetilde{G}_{2}\right)^{\cdot \frac{n}{2}}\right|$ (say for $n$ even) and obtains the following inequality:

$$
F_{\circ}\left(n ; \Gamma, G_{1} \cup \widetilde{G}_{2}\right) \geq \frac{1}{18} G_{\circ \circ}\left(\frac{n}{8}\right) \cdot \frac{1}{4} F_{\circ}\left(\frac{n}{4} ; \Gamma_{2}, G_{2}\right) .
$$

For instance, polycyclic groups, e.g. nilpotent non-virtually Abelian groups $\Gamma$, have distorted normal subgroups, but the above inequality fares no better than $\left(F_{\circ}(n) \geq\right.$ $\frac{1}{2} G_{\circ}\left(\frac{n}{2}\right)$ in (all?) these cases and (reasonably) sharp isoperimetric inequalities for "distorted normal extensions" remain problematic.

Distortion and isoperimetry of homogeneous spaces. Let $\Gamma_{1} \subset \Gamma$ be an arbitrary (not necessarily normal) subgroup. Then the isoperimetric profile of the homogeneous $\Gamma$-space $X=\Gamma / \Gamma_{1}$ is bounded by the profile of $\Gamma$. If we want to take into account the profile and the distortion of $\Gamma_{1}$ we may use the action of $\Gamma \times \Gamma_{1}$ on $X_{1}=\Gamma$, where $\Gamma$ acts from the left and $\Gamma_{1}$ from the right. Then the above inequality remains valid,

where observe that

$$
F_{\circ}\left(n ; X_{1}\right) \geq \frac{1}{4} F_{\circ}\left(\frac{n}{4} ; X\right) \cdot \frac{1}{4} G_{\circ}\left(\frac{n}{4} ; \Gamma_{1} \subset \Gamma\right),
$$

$$
F_{\circ}\left(n ; X_{1}\right) \leq F_{\circ}\left(n ; \Gamma \times \Gamma_{1}\right) \leq F_{\circ}(n ; \Gamma) \cdot F_{\circ}\left(n ; \Gamma_{1}\right) .
$$

Question. What is the (exact if possible) relation between the isoperimetric profiles of a group $\Gamma$ and of this $\Gamma$ regarded as the homogeneous space under the right/left action of $\Gamma \times \Gamma$ ?

\section{Entropy}

Let $(X, \lambda)$ be a Borel measure space, where $\lambda$ is regarded as a background measure and where we use the notation $|Y|=|Y|_{\lambda}=\lambda(Y)$ for all $Y \subset X$.

The basic examples are given by countable spaces $(X, \lambda)$ with the unitary measures, where all atoms have unit weights (thus, $|Y|=\operatorname{card}(Y)$ ), and by the Euclidean spaces with the Lebesgue or with the Gaussian measures $\lambda$.

Consider measures $\mu=f(x) \lambda$ for (non-strictly) positive measurable functions $f$ on $X$ and first define the entropy of such a $\mu$ where $f(x)$ is constant on its (essential) support $S=\operatorname{supp}(f) \subset X$ by

$$
\operatorname{ent}_{\lambda}(\mu)=\log |S|=\log (\mu(S))-\mu(S)^{-1} \int_{S} \log (f) d \mu,
$$


where, observe, $f \equiv \mu(S) /|S|$.

Then, for a general $\mu=f \lambda$, let $|\mu|_{\varepsilon}=\lambda_{\varepsilon}(\mu)$ denote the infimum of the $\lambda$ measures of the subsets $S_{\varepsilon} \subset X$ with $\mu\left(S_{\varepsilon}\right) \geq(1-\varepsilon) \mu(X)$, where we assume that $\mu$ has finite total mass, $|\mu|:=|X|_{\mu}=\mu(X)<\infty$.

Take the Cartesian (tensorial) powers $\left(X^{N}, \lambda^{N}=\lambda^{\otimes N}, \mu^{N}=\mu^{\otimes N}\right)$ and with $\lambda^{\otimes N}$ for the background measures on $X^{N}$. Set

$$
\left.\operatorname{ent}\left(\mu^{N}-[\varepsilon]\right)=\liminf _{N \rightarrow \infty} \frac{1}{N} \log \left|\mu^{N}\right|_{\varepsilon}\right)
$$

and

$$
\operatorname{ent}(\mu)=\operatorname{ent}_{\lambda}(\mu)=\lim _{\varepsilon \rightarrow 0} \operatorname{ent}\left(\mu^{N}-[\varepsilon]\right) .
$$

Observe that this entropy is invariant under scaling of $\mu$, that is, $\operatorname{ent}(c \cdot \mu)=\operatorname{ent}(\mu)$, while ent $c_{\lambda}=$ ent $_{\lambda}+\log (c)|\mu|$.

If $\mu$ is a probability measure with a $\lambda$-measurable density function $f=d \mu / d \lambda$ and with the support denoted $S \subset X$, then $\operatorname{ent}_{\lambda}(\mu) \leq \log \lambda(S)$ with equality (only) for $\mu=\lambda(S)$. On the other hand, $\operatorname{ent}_{\lambda}(\mu) \geq \log \left(\sup _{x \in S} f(x)\right)-1$.

We shall use the above definition only for $\log -L L N$-measures $\mu$, i.e., where $\mu=$ $f \lambda$ for a $\lambda$-measurable function $f$ such that $\log (f)$ satisfies

The law of large numbers. The $\mu^{\otimes N}$ measure of the subset $Y(\varepsilon, N) \subset X^{N}$ of the points $y \in X^{N}$, where

$$
\frac{1}{N}\left|\log \left(f^{\otimes N}(y)\right)-\int_{S} \log (f) d \mu\right| \geq \varepsilon
$$

satisfies

$$
\mu^{\otimes N}(Y(\varepsilon, N)) \rightarrow 0 \text { for } N \rightarrow \infty .
$$

One knows that (LLN) is satisfied if and only if the function $|\log (f)|$ is $\mu$ summable on its support $S$, e.g., if $|\log (f)|$ is bounded on $S$.

If $\mu$ is not log-LLN, one can LLN-regularize it, e.g., by cutting away the part of the support of $f$ where $|\log (f)|$ approaches infinity and then define a suitable regularized entropy with such an approximation.

Cartesian additivity of the entropy. Observe that LLN ensures the additivity of the entropy under the Cartesian product of measure spaces and yields the celebrated

Boltzmann formula. All $\log -L L N$-measures $\mu$ satisfy

$$
\operatorname{ent}_{\lambda}(\mu)=\log |\mu|-|\mu|^{-1} \int_{S} \log (f) d \mu=\log |\mu|-|\mu|^{-1} \int_{S} f \log (f) d \lambda
$$

( for $|\mu|$ denoting the total mass $\mu(X)=\mu(S)$ ). 
In other words,

the $\mu$-average of $\log (f)$ plus $\operatorname{ent}_{\lambda}(\mu)$ equals the $\log$ of the total mass of $\mu$.

In particular, the entropy of a probability measure $\mu$ is expressed by the Boltzmann integral,

$$
\operatorname{ent}(\mu)=\int_{S} \log \frac{1}{f} d \mu=\int_{S} f \log \frac{1}{f} d \lambda .
$$

This formula is customarily taken for the definition of the entropy without assuming LLN, but only the convergence of the Boltzmann integral, possibly to $\pm \infty$. This definition is equivalent to the above "regularized entropy", but in all our applications we can (and do) assume that $\mu$ is log-LLN.

5.1. Hölder inequality via tensorisation. We introduce below the Gibbs tensorisation trick and then use it for the proof of the Shannon inequalities relating the entropy of a measure and its push-forwards under the maps (partitions) in a given family.

Hölder inequality. The log of the integral

$$
\int_{X} \prod_{i \in I} f_{i}(x)^{\beta_{i}} d x
$$

is a convex function of $\bar{\beta}=\left\{\beta_{i}\right\} \in \mathbb{R}^{I}$ for arbitrary positive functions $f_{i}$ on $X$.

Proof. The inequality

$$
\log \left(\int_{X} \prod_{i \in I} f_{i}(x)^{\alpha_{i} \beta_{i}}\right) d x \leq \sum_{i \in I} \alpha_{i} \log \left(\int f_{i}(x)^{\beta_{i}}\right)=\log \prod_{i \in I}\left(\int f_{i}(x)^{\beta_{i}}\right)^{\alpha_{i}}
$$

for $\sum_{i} \alpha_{i}=1, \alpha_{i} \geq 0$, is (trivially) true if the functions $f_{i}(x)$ are constant on the intersection $S \subset X$ of their supports (with the equality for functions with a common support $S \subset X$, where all $f_{i}$ are constant). The general case reduces to this by the law of large numbers via the tensorisation.

This argument also shows by how much the inequality deviates from equality.

$$
\begin{aligned}
& \text { Denote } \mu_{i}=g_{i} d x \text { for } g_{i}=f_{i}^{\beta_{i}} \text { and let } \mu=\left(\prod_{i \in I} g_{i}^{\alpha_{i}}\right) d x \text {. Then } \\
& \begin{aligned}
\log \int \prod_{i \in I} g_{i}^{\alpha_{i}} d x & \leq \sum \alpha_{i} \operatorname{ent}_{\mu_{i}}(\mu) \\
& \leq \sum \alpha_{i} \log \left(\mu_{i}(X)\right)=\log \prod_{i \in I}\left(\int_{X} g_{i} d x\right)^{\alpha_{i}} .
\end{aligned}
\end{aligned}
$$

The Hölder inequality can be equivalently stated as follows: 
Let $v$ be a measure on the linear space $X$, and let $Y$ be the linear dual to $X$. Then the function

$$
\Psi(y)=\Psi_{\nu}(y)=\log \left(\int_{X} \exp \langle x, y\rangle d \nu\right)
$$

is convex on $Y$, where the entropy of a measure with the density function $\exp (\varphi(x)), x \in \mathbb{R}$, equals the derivative $\psi^{\prime}(y=1)$ for $\psi(y)=\int_{\mathbb{R}} \exp \langle x, y\rangle d x$ by the Boltzmann formula.

This appears in the Gibbsian thermodynamics as the concavity of the entropy of the ideal gas and represents a tiny instance of Boltzmann's and Gibbs' ideas (see [20]).

Remarks. (a) The information-theoretic rendition of the Gibbs argument is often presented as a chat between Alice and Bob. (See [11] and references therein.)

(b) The differential $D \Psi: Y \rightarrow X$ injectively sends $Y$ to $X$, where

the closure of the image equals the convex hull of the support of $\mu$.

Thus, if $X=Y=\mathbb{R}^{n}$, then the volume of this hull equals the integral of the determinant of the Hessian of the (convex!) function $\Psi$, where the $\mathbb{R}_{+}$-valued map

$$
\Psi \mapsto M(\Psi):=\int_{Y} \operatorname{det}(\operatorname{Hess}(\Psi(y))) d y
$$

obeys non-trivial convexity relations: the Minkovski inequality, $M^{\frac{1}{n}}\left(\Psi_{1}+\Psi_{2}\right) \geq$ $M^{\frac{1}{n}}\left(\Psi_{1}\right)+M^{\frac{1}{n}}\left(\Psi_{2}\right)$, and the Alexandrov-Fenchel-Hodge inequality. (See [13] for a survey and references.)

5.2. Entropic profiles and stable $\boldsymbol{E}_{\mathrm{o}}$ functions of families of partitions. Given a finite mass measure $\mu$ on a Borel measure space $X=(X, \lambda)$ with a family of partitions $P_{i}$, we denote by $\mu_{i}=\mu / P_{i}=$ the push-forward of $\mu$ to $X_{i}=X / P_{i}$, call this the $P_{i}$-reduction of $\mu$, and write

$$
\operatorname{ent}\left(\mu / P_{i}\right)=\operatorname{ent}\left(\mu_{i}\right)=\operatorname{ent}_{\lambda_{i}}\left(\mu_{i}\right)
$$

for the background measures $\lambda_{i}$ in $X_{i}$.

For example, if $\mu$ equals the restriction of the background measure $\lambda$ on $X$ to a subset $Y \subset X$, then the value of the density function of $\mu_{i}$ with respect to the background measure $\lambda_{i}$ on $X / P_{i}$ at each point $x_{i} \in X / P_{i}$ equals the Fubini mass of the corresponding $P_{i}$-slice of $Y$.

Denote by $\mu_{x_{i}}, x_{i} \in X_{i}=X / P_{i}$, the measure $f d P(x)$ on the slice $P^{-1}\left(x_{i}\right)$ for the background Fubini measure $d P(x)$ on this slice and $f=d \mu / d \lambda$, and let ent $x_{i}$ be 
the entropy of $\mu_{x_{i}}$ with respect to $d P(x)$ on this slice. Define the entropy of $(X, \mu)$ over $X_{i}$, also denoted ent $\left(P_{i}\right)$, as the average

$$
\operatorname{ent}\left(P_{i}\right)=\mu(X)^{-1} \int_{X_{i}} \text { ent }_{x_{i}} d \mu_{i} .
$$

It is obvious (but significant) that

Entropy is additive.

$$
\operatorname{ent}(P)+\operatorname{ent}(\mu / P)=\operatorname{ent}(\mu)
$$

Finite example. Let $P$ be a partition of $X$, a finite set with the unitary atoms, and take a subset $Y \subset X$. Denote by $|P(y)|$ the cardinality of the $P$-slice of $Y$ through $y \in Y$, and observe with the Boltzmann (and Shannon in the finite case) formula that

$$
\operatorname{ent}(P \mid Y)=\log \prod_{y \in Y}|P(y)|^{\frac{|P(y)|}{|Y|}} \text {. }
$$

Entropic profile. Consider a family $\mathcal{P}$ of partitions $P_{i}, i \in I$, of $X$, where we usually assume that the single slice partition, corresponding to the map of $X$ to a single point, is among our $P$. Every LLN measure $\mu$ on $X$ defines the point $e(\mu)=\left\{\operatorname{ent}\left(P_{i}\right)\right\} \in \mathbb{R}^{I}$; the set $\operatorname{ENT}(\mathcal{P})$ of these points for all $\mu$ is called the entropic profile of $\mathcal{P}$. In what follows we shall evaluate the conical convex hull of $\operatorname{ENT}(\mathcal{P}) \subset \mathbb{R}^{I}$ in the simple cases.

The definition of the entropy and the slice removal lemma from 4.4 imply the following

Sliced tensorisation lemma. Given a finite family $\mathcal{P}$ of partitions $P_{i}, i \in I$, of $X$ and an $L L N$ measure $\mu$ on $X$, there exists, for every $\varepsilon>0$, an integer $N_{0}=N_{0}(\varepsilon, \mu, \mathcal{P})$ and a subset $Y=Y_{N}$ in the Cartesian power $X^{N}$, for every $N \geq N_{0}$, such that

$$
Y \subset \operatorname{supp}\left(\mu^{\otimes N}\right), \quad \text { where } \mu^{\otimes N}(Y) \geq(1-\varepsilon) \mu^{\otimes N}(X),
$$

and the Fubini measures $\phi_{i}^{N}=\lambda^{\otimes N} / \lambda_{i}^{\otimes N}$ of the $P_{i}^{N}$-slices of $Y$ satisfy

$$
N\left(\operatorname{ent}\left(P_{i}^{N}\right)-\varepsilon\right) \leq \log \left(\phi_{i}^{N}\left(P_{i}^{N}(y) \cap Y\right)\right) \leq N\left(\operatorname{ent}\left(P_{i}^{N}\right)+\varepsilon\right)
$$

for all $y \in Y$ and all $i \in I$.

Next observe that the $E_{\mathrm{o}}$-functions of Cartesian powers of partitions $P_{i}$ of $X$, satisfy,

$$
E_{\circ}\left(n_{i}^{N_{1}} ; P_{i}^{N_{1}}\right) \cdot E_{\circ}\left(n_{i}^{N_{2}} ; P_{i}^{N_{2}}\right) \geq E_{\circ}\left(n_{i}^{N_{1}+N_{2}} ; P_{i}^{N_{1}+N_{2}}\right)
$$

and define

$$
E_{\infty}\left(n_{i} ; P_{i}\right)=\lim _{N \rightarrow \infty}\left(E_{\circ}\left(n_{i}^{N} ; P_{i}^{N}\right)\right)^{\frac{1}{N}} .
$$

The above lemma implies the following 
Shannon $\boldsymbol{E}_{\infty}$-inequality. Let $\mathcal{P}=\left\{P_{i}\right\}$ be a finite family of partitions on $X$ and $\mu$ a measure of finite mass on $X=(X, \lambda)$. Then the entropies $\operatorname{ent}\left(P_{i}\right)=\operatorname{ent}(\mu)-$ $\operatorname{ent}\left(\mu / P_{i}\right)$ of $P_{i}$ with respect to $\mu$ satisfy

$$
\operatorname{ent}(\mu) \geq \log E_{\infty}\left(\exp \left(\operatorname{ent}\left(P_{i}\right)\right) ; \mathcal{P}\right) .
$$

Remark on Hölder. The tensorisation lemma also implies the Hölder version of the above inequality.

Let $f_{i} \geq 0$ be measurable functions on $X_{i}=X / P_{i}$, let

$$
\left|f_{i}\right|_{p}=\left(\int_{\operatorname{supp}\left(f_{i}\right)} f_{i}^{p}\right)^{\frac{1}{p}}
$$

and let $\left|\Pi_{\mathcal{P}} f_{i}\right|_{1}$ denote the integral of the product of the pullbacks of $f_{i}$ to $X$.

Then

$$
\left|\Pi_{\mathcal{P}} f_{i}\right|_{1} \geq E_{\infty}\left(\left|\Pi_{\mathcal{P}} f_{i}\right|_{1} /\left|f_{i}\right|_{p_{i}} ; \mathcal{P}\right)
$$

for all $\left\{p_{i}\right\} \in \mathbb{R}_{+}^{I}$.

If all $P_{i}$ are single slice partitions, this reduces to the Hölder inequality from 5.1 with positive $p_{i}$ (and with no entropic correction term).

5.3. Shannon inequalities for the coordinate line and plane partitions. Let $(X, \lambda)=\mathrm{X}_{i}\left(X_{i}, \lambda_{i}\right), i=1,2, \ldots, k$. Then the partitions $P_{i}$ of $X$ into the "coordinate lines" with the slices isomorphic to $X_{i}$ and corresponding to the projections $P_{i}: X \rightarrow X_{\hat{i}}=\left(X_{\hat{i}}, \lambda_{\hat{i}}\right)=\times_{j \in I \backslash\{i\}}\left(X_{j}, \lambda_{j}\right)$ satisfy

$$
\operatorname{ent}(\mu) \geq \sum_{i} \operatorname{ent}\left(P_{i}\right)
$$

or, equivalently,

$$
\operatorname{ent}(\mu) \leq \frac{1}{k-1} \sum_{i} \operatorname{ent}\left(\mu / P_{i}\right)
$$

for all measures $\mu$ on $X$. Furthermore, the partitions $P_{J}$ of $X$ into the fibers of the projections $X=X_{I} \rightarrow X_{I \backslash J}=\times_{i \in I \backslash J} X_{i}$ (with "J-plane" slices representing $\left.X_{J}=\times_{i \in J} X_{i}\right)$ satisfy

$$
\operatorname{ent}(\mu) \geq \sum_{J \subset I} \alpha_{J} \cdot \operatorname{ent}\left(P_{J}\right)
$$

for all partitions of unity $\alpha_{J}$ of I (see 4.3).

Proof. Here, obviously, $E_{\infty}=E_{\circ}$ and the above applies. 
Loomis-Whitney inequality. This is an upper bound on $|Y|=\lambda(Y)$ for subsets $Y \subset X$ in terms of the background measures of $Y / P_{i}$, (assuming these are measurable) written as if it were a lower bound,

$$
|Y| \geq \prod_{i}|Y|\left(\lambda_{\hat{i}}\left(Y / P_{i}\right)\right)^{-1} .
$$

This follows from the Shannon inequality, since ent $\left.\lambda_{\hat{i}}\left(\mu_{i}\right)\right) \leq \lambda_{\hat{i}}\left(\operatorname{supp}\left(\mu_{i}\right)\right)$ and $\operatorname{ent}(\mu)=\log (\lambda(Y))$ for $\mu=\lambda \mid Y$.

Similarly one derives the Shearer inequality that is the bound on $\log |Y|$ by $\log |Y|-\log \left(\lambda_{\hat{J}}\left(Y / P_{J}\right)\right.$ substituting $\operatorname{ent}\left(P_{J}\right)$ in $\mathrm{Sh}_{\alpha}$. (The role of the entropy in such inequalities was pointed out to me by Noga Alon.)

If $X_{i}$ are countable sets with the atoms of unit weights, then the Shannon inequality for subsets $Y \subset X=\times_{i} X_{i}$ with the restricted product unitary measures reads,

Combinatorial Shannon inequality for the coordinate line partitions. Let $\left|P_{i}(y)\right|$, $y \in Y$, denote the cardinality of the $P_{i}$-slice of $Y$ through $y$. Then the geometric means

$$
\left|M P_{i}\right|=\left(\prod_{y \in Y}\left|P_{i}(y)\right|\right)^{\frac{1}{|Y|}}
$$

satisfy

$$
\prod_{i}\left|M P_{i}\right| \leq|Y|
$$

Harper inequality. The Shannon inequality, when applied to the vertex set $X$ of the edge graph of a Euclidean $n$-cube with the edges for slices, says that

the vertex and the edge numbers of every subgraph $Y$ in the cubical graph satisfy

$$
N_{\text {vert }} \geq 4^{N_{\text {edg }} / N_{\text {vert }}} \text {. }
$$

For example, if all vertices in $Y$ have the valency (degree) at least $d$, then $|Y| \geq 2^{d}$.

Another corollary of the combinatorial Shannon inequality is the following (well known) relation between the three numbers: the cardinality $|Y|$, the number $N$ of the slices of $Y$ with respect to all $P_{i}$ and the sum $C$ of the cardinalities of all these slices.

$A^{B}$-inequality. Let $A=C / N$ and $B=C /|Y|$. Then

$$
|Y| \geq A^{B} .
$$

Proof. Since the function $s^{s}$ is $\log$-convex, $\log \left(s^{s}\right)^{\prime \prime}=1 / s$,

$$
A^{B} \leq \prod_{S}|S|^{|S|} \leq|Y|,
$$

where the product is taken over all slices $S$ of the partitions $P_{i}$. 
5.4. Strict concavity of the entropy and refined Shannon inequalities. A probability measure $\mu$ on $X_{1} \times X_{2}$ can be regarded as a family of probability measures $\mu_{x_{1}}$ on $X_{2}$ parametrized by $x_{1} \in X_{1}$, where the density $f_{x_{1}}\left(x_{2}\right)$ of (almost) every measure $\mu_{x_{1}}$ on $X_{2}$ equals the restriction of the density of $\mu$ to $x_{1} \times X_{2} \subset X_{1} \times X_{2}$ divided by $p\left(x_{1}\right)=\int_{X_{2}} f_{x_{1}}\left(x_{2}\right) d \lambda_{2}$.

The Shannon inequality written as $\operatorname{ent}\left(\mu / P_{2}\right) \geq \operatorname{ent}\left(P_{1}\right)\left(=\operatorname{ent}(\mu)-\operatorname{ent}\left(\mu / P_{1}\right)\right)$ says that the entropy is a concave function on the space of probability measures on $X_{2}$, since the measure $\mu_{2}$ on $X_{2}$, that is, the push-forward of $\mu$, equals the $p\left(x_{1}\right)$ weighted convex combination of the probability measures $\mu_{x_{1}}$, while the entropy is (defined as) the corresponding convex combination of the entropies of $\mu_{x_{1}}$.

In fact, the entropy is strictly concave as follows from the Boltzmann formula and the strict convexity of the function $t \cdot \log (t)$. (This is the common way for deriving the Shannon inequality.) Then the quantity ent $(\mu)-\operatorname{ent}\left(P_{1}\right)-\operatorname{ent}\left(P_{2}\right) \geq 0$ tells us how far $\mu$ is from equilibrium, i.e., a probability measure $\mu^{\prime}$ on $X_{1} \times X_{2}$, for which the probability measures $\mu_{x_{1}}^{\prime}$ on $X_{2}$ are mutually equal for all $x_{1} \in X_{1}$, or equivalently all $\mu_{x_{2}}^{\prime}$ on $X_{1}$ are equal.

Here is another characteristic of (non-)equilibrium for measures $\mu$ on product spaces $X=\times_{i} X_{i}, i \in I$.

The index set $I \sqcup I$ (disjoint union of $I$ with itself), and, hence, the Cartesian power $X^{2}$ of $X$, is naturally acted upon by the Mendelian recombination group $\mathbb{Z}_{2}^{I}=$ $(\mathbb{Z} / 2 \mathbb{Z})^{I}$, which is generated by $|I|$ coordinate involutions on $I \sqcup I$ and/or on $X_{i} \times X_{i}$ for all $i \in I$. By strict convexity, a measure $\mu$ on $X$ is at equilibrium, where (by definition if you wish) all Shannon inequalities $\mathrm{Sh}_{\alpha}$ become equalities if and only if the measure $\mu^{\otimes 2}$ on $X^{2}$ is invariant under $\mathbb{Z}_{2}^{I}$ (and where $\mu^{\otimes 2}$ is invariant under the diagonal involution on $X^{2}$ for all $\mu$ on $X$ ).

We introduce the entropic displacement of $\mu^{\otimes 2}$ by $z$,

$$
\left|\mu^{\otimes 2}-z\left(\mu^{\otimes 2}\right)\right|_{\text {ent }}:=\operatorname{ent}\left(\frac{1}{2}\left(\mu^{\otimes 2}+z\left(\mu^{\otimes 2}\right)\right)-\frac{1}{2}\left(\operatorname{ent}\left(\mu^{\otimes 2}\right)+\operatorname{ent}\left(z\left(\mu^{\otimes 2}\right)\right)\right) \geq 0,\right.
$$

and then identify involutions $z \in \mathbb{Z}_{2}^{I}$ with subsets $J \subset I$ by

$$
z \leftrightarrow J=J(z)=\operatorname{supp}(z) \subset I,
$$

where the support of $z$ is defined by $z(i) \neq i$.

The composition of involutions corresponds to the symmetric difference of subsets which we denote $J_{1} \cdot J_{2}:=\left(J_{1} \cup J_{2}\right) \backslash\left(J_{1} \cap J_{2}\right)$. We also abbreviate by writing

$$
|J|_{\mathrm{ent}}(\mu)=\left|\mu^{\otimes 2}-z(J)\left(\mu^{\otimes 2}\right)\right|_{\mathrm{ent}},
$$

where $|J|_{\text {ent }}(\mu)=\left|J^{\perp}\right|_{\text {ent }}(\mu)$ for $J^{\perp}=I \backslash J$.

A measure $\mu$ on $X$ satisfies the equality

$$
\operatorname{ent}\left(P_{J}\right)+\operatorname{ent}\left(P_{J^{\perp}}\right)=\operatorname{ent}(\mu)
$$


if and only if $\mu^{\otimes 2}$ is $z(J)$ - (or, equivalently, $z\left(J^{\perp}\right)$ )-invariant; this is also equivalent to

$$
|J|_{\text {ent }}(\mu)=0 \text {. }
$$

Since the entropy is strictly concave, the function $|J|_{\text {ent }}(\mu)$ of $J \subset I$ satisfies some triangle-type inequalities,

$$
\left|J_{1} \cdot J_{2}\right|_{\mathrm{ent}}(\mu) \leq \Delta_{\mu}\left(\left|J_{1}\right|_{\mathrm{ent}}(\mu),\left|J_{2}\right|_{\mathrm{ent}}(\mu)\right),
$$

where $\Delta_{\mu}(0,0)=0$ for all $\mu$ and $\Delta_{\mu}(a, b)$ is uniformly continuous in $(a, b)$ with the modulus of continuity $\delta$ depending on $\mu$. Moreover, $\delta$ is uniformly bounded on certain (compact in a suitable sense) classes of measures $\mu$.

For example, if the density function $f$ of $\mu$ satisfies

$$
\int_{X}|\log (f(x))| d \mu \leq \text { const }<\infty,
$$

then $\delta$ is bounded by some universal $\delta_{\text {const }}$, as a simple continuity argument shows.

This is useful, for instance, if $\log (f(x)) \leq 0$, e.g., if $X$ is a discrete space with unitary atoms, where $(\Delta)$ becomes a relation between the entropies of $P_{J}$ depending only on ent $(\mu)$,

$$
\left|J_{1} \cdot J_{2}\right|_{\text {ent }}(\mu) \leq \Delta_{\text {ent }(\mu)}\left(\left|J_{1}\right|_{\text {ent }}(\mu),\left|J_{2}\right|_{\text {ent }}(\mu)\right)
$$

for some function $\Delta_{e}(a, b)$ which is continuous in $a, b$ and $e$ such that $\Delta_{e}(0,0)=0$.

Remarks. (a) All this is, apparently, well known, but I could not find a reference; nor do I know a specific sufficiently "elegant" $\Delta_{e}(a, b)$. I guess that there are sharp "mixed symmetric mean inequalities" for measures on $\times X_{i}$ similar to the classical Muirhead's inequalities, such as the mixed discriminant inequality of Alexandrov (which is GL $(k)$ - rather than just $S_{k}$-symmetric).

(b) The above generalizes to Cartesian powers $X^{N}$ with the Cartesian products of $I$-copies of the permutation group $S_{N}$ acting on it. The resulting inequalities become, in a sense, asymptotically sharp for $N \rightarrow \infty$ due to the law of large numbers (applied to convolution of measures on the spaces of measures).

A possible framework for this is suggested by the Mendelian dynamics on the space of measures on the disjoint union $X^{\sqcup}=\sqcup_{N} X^{N}$ of the Cartesian powers of $X=\mathrm{X}_{i \in I} X_{i}$, where $X^{N}=\times_{i} X_{i}^{N}$ and where this equality is defined (only) up to the action of the Cartesian power of the permutation group, $\left(S_{N}\right)^{I}$ acting on $X^{N}$. Every partition of unity $\alpha_{J}$ where all $\alpha$ are rational with the common denominator, say $\alpha_{J}=n_{J} / d$, defines a bijection (equality) $\times_{J} X_{J}^{n_{J}}=X^{d}$ for $X_{J}=\times_{i \in J} X_{i}$ and similarly the equalities $\times_{J} X_{J^{N}}^{n_{J}}=X^{d N}$ for all $N$.

The Mendel map $F$ sends measures $\mu_{N}$ on $X^{N}$ to measures $F\left(\mu_{N}\right)$ on the tensor products of the $J^{N}$-reductions of $\mu_{N}$ to $X_{J^{N}}$ for all subsets $J \subset I$. This $F$ is accompanied by the power map $\mu_{N} \mapsto \mu^{\otimes d}$ which should be thought of as "multiplication 
by the scalar $=d "$ in some kind of log of the tensor algebra. Then the "ideal $d$-th root" $F^{\otimes \frac{1}{d}}$ of the Mendel map apparently (I did not check this) exponentially contracts (in a suitable metric) the space of "graded measures" $\left\{\mu_{N}\right\}$ to the subspace of the equilibrium (i.e., tensor product) measures (as in the Mendel-Robbins-Geiringer theorem, see [17] and references therein) and satisfies $\frac{1}{d} \operatorname{ent}\left(F\left(\mu_{N}\right)\right) \geq \operatorname{ent}(\mu)$ in agreement with the Shannon-Shearer inequality.

Question. What is a comprehensive formalism that would fully reflect the $\left(S_{N}\right)^{I}$ symmetry along with the $N$-grading and would also embrace the Brascamp-Lieb and the Lieb-Ruskai inequalities below?

(c) The Hessian of the entropy on the space (affine simplex) $M$ of probability measures, being positive definite by the Shannon inequality, defines a (non- complete) Riemannian metric on $M$, called Fisher-Rao-Kramer (Antonelli-Strobeck, Svirezhev-Shahshahani, Karlquist) metric, (also underlying the Einstein-Onsager relations as was explained to me by Alexander Gorban) that, by the Boltzmann formula, equals the spherical metric induced by the log-radial (compare 2.2) projection $\left(p_{i}\right) \mapsto\left(\sqrt{p_{i}}\right)$ of the unit $n$-simplex $\Delta_{n} \subset \mathbb{R}^{n+1}$ of probability measures to the unit sphere $S^{n} \subset \mathbb{R}^{n+1}$.

The meaning of $\left(p_{i}\right) \mapsto\left(\sqrt{p_{i}}\right)$ becomes clearer with the the moment map

$$
\mathbb{C}^{n+1} \rightarrow \mathbb{C}^{n+1} / \mathbb{T}^{n+1}=\mathbb{R}_{+}^{n+1} \supset \Delta_{n}
$$

for the coordinate-wise (Hamiltonian!) action of the torus $\mathbb{T}^{n+1}$ on $\mathbb{C}^{n+1}$, where the Riemannian quotient metric on $\mathbb{R}_{+}^{n+1}=\mathbb{C}^{n+1} / \mathbb{T}^{n+1}$ identifies with the Hessian of $\sum p_{i} \log \left(p_{i}\right)$ on $\mathbb{R}_{+}^{n+1} \supset \Delta_{n}$.

Questions. Is there a geometric picture where this unitary symmetry of the Fisher metric is seen simultaneously with the tensorisation property (definition) of the entropy?

What are other "entropy type" functions associated to Kähler manifolds with isometric actions of compact groups?

5.5. On sliced spaces. A sliced set is a set $X$ with a distinguished family $8 \subset 2^{X}$ of subsets $S \subset X$, called slices. More generally "a slicing" of a measure space $X$ is given by a measurable map $\widetilde{X} \rightarrow \underset{\widetilde{P}}{X}$, where $\widetilde{X}$ is a measure space with a distinguished partition $\widetilde{P}$, where the map $\widetilde{X} \rightarrow X$ is one-to-one on (almost) every slice of $\widetilde{P}$. (Alternatively, a sliced structure is a measure on $2^{X}$ with some regularity to it.)

Here is a generalization of the combinatorial Shannon inequality for pairs of partitions to sliced spaces.

No- $\Delta$ inequality. Let $X$ be sliced by subsets $S \in 8 \subset 2^{X}$ such that 
(a) every point of $X$ is contained in at most two slices,

(b) every two non-equal slices intersect at a single point if at all.

If $\&$ contains no triple of pairwise intersecting slices, then every finite subset $Y$ in $X$ satisfies the combinatorial Shannon inequality

$$
|Y|^{|Y|} \geq \prod_{S \in S}|Y \cap S|^{|Y \cap S|} .
$$

Proof. Consider the 2-chains $\left(y_{0}, S_{1}, y_{1}, S_{2}, y_{2}\right)$ in $Y$, where the pairs of points $\left(y_{i-1}, y_{i}\right)$ are contained in the slices $S_{i}$ for $i=1,2$ and where $S_{1} \neq S_{2}$.

The geometric mean $G_{0}$ of the number of such chains over $y_{0} \in Y$, i.e, the geometric mean of the number $N_{0}(y)$ of the chains starting at $y \in Y$ is $\geq$ than the geometric mean $G_{1}$ of the number $N_{1}\left(y_{1}\right)=\left|S_{1}\right| \cdot\left|S_{2}\right|$ of the chains with the middle point $y_{1} \in Y$ by the geometric-arithmetic mean inequality.

Since the number of the chains between every pair of points $\left(y_{0}, y_{2}\right)$ in $X$ is $\leq 2$ by our assumptions on $\delta$, we get our inequality

$$
|Y| \geq\left(\prod_{y} N_{0}(y)\right)^{\frac{1}{|Y|}}=G_{0} \geq G_{1}=\left(\prod_{y} N_{1}(y)\right)^{\frac{1}{|Y|}}=\left(\prod_{S \in S}|Y \cap S|^{|Y \cap S|}\right)^{\frac{1}{|Y|}} .
$$

Questions. What of this kind would generalize Shannon for more than two partitions? What are further "chain uniqueness" and/or "no short cycle" conditions leading to further inequalities? In particular, if a certain co-entropic inequality holds for all subsets $Y$ of cardinality $\leq N$, for large $N$ depending on the type of the inequality, does it then hold for all finite subsets $Y$ ? Is Gibbs' tensorisation helpful for such questions?

Notice in this regard that the entropic profile of a sliced space is monotone decreasing under slice-wise injective maps $\psi: X_{1} \rightarrow X_{2}$. In fact, a simple computation shows that the decrease of the entropy under $\psi$ is greater than the average entropic decreases of the slices of the two spaces for all measures $\mu$ on $X_{1}$,

$$
\operatorname{ent}(\mu)-\operatorname{ent}\left(\psi_{*}(\mu)\right) \geq \sum_{S_{1} \in \delta_{1}} \operatorname{ent}\left(\mu \mid S_{1}\right)-\sum_{S_{2} \in \mathcal{S}_{2}} \operatorname{ent}\left(\psi_{*}\left(\mu \mid S_{2}\right) .\right.
$$

Remark. There are many homogeneous sliced spaces $X$, where one would like to know the relation between the cardinalities of subsets $Y \subset X$ and their slices, but a comprehensive answer may be out of reach.

For example, some relations of this kind in affine and projective spaces $X$ sliced by their subspaces appear in combinatorial number theory, e.g., in the van der Waerden and the Szemerédy theorems on arithmetic progressions, where one uses something more complicated than the Shannon inequality. 
Also many (especially homogeneous and symmetric) Riemannian manifolds are naturally sliced by some families of submanifolds, e.g., by geodesics (when these are properly embedded) and by higher dimensional (geodesic) submanifolds. The problem of determining the co-entropic (as well as isoperimetric) profiles for these "slicings" remains open in most cases.

$\mathcal{P}^{N}$-tensorisation. One can slightly improve the $E_{\infty}$-ent-relation by taking into account the following.

1. The Cartesian power $\mathcal{P}^{N}$ of the family of partitions $P_{i}, i \in I$, consists of $|I|^{N}$ partitions indexed by maps $\{1,2, \ldots, N\} \rightarrow I$ (our definition of $E_{\infty}$ takes into account only the $|I|$ partitions $P_{i}^{N}$ corresponding to the constant maps), where the set $I^{N}$ is naturally acted upon by the permutation group $S_{N}$ and where the orbits of the corresponding partitions of $X^{N}$ are regarded as monomials $\prod P_{i}^{N_{i}}$ over all $\left\{N_{i}\right\}$ with $\sum N_{i}=N$.

2. The fully tensorised $E_{\mathrm{o}}$-function is submultiplicative, while the entropies of partitions are multiplicative.

With this in mind, we define $E_{\otimes}\left(L_{i}\right) \geq E_{\infty}\left(L_{i}\right)$ as we did for $E_{\infty}$, except that now we take the subsets $Y \subset X^{M \cdot N}$, where the slices of the partitions represented by $\left(\prod P_{i}^{M_{i}}\right)^{N}$ have the Fubini measures bounded from below by $\left(\prod L_{i}^{M_{i}}\right)^{N}$ for all monomials $\prod P_{i}^{M_{i}}$, and take the maximal function $E_{\otimes}$ that minorizes the measures of all such $Y$ for $M, N \rightarrow \infty$ with $N \gg M$.

(The smaller the class of subsets $Y$ we use, the greater is the resulting $E_{\circ}$-function, where one may further limit the class of $Y$ by requiring the symmetry of $Y \subset X^{N}$ under the $S_{N}$-action. On the other hand, if one limits the class of competing $E$, then the outcome becomes smaller. Yet, one loses little in the present context if one maximizes over all convex functions $E$.)

Then we see, as before, that

$$
\operatorname{ent}(\mu) \geq \log E_{\otimes}\left(\exp \left(\operatorname{ent}\left(P_{i}\right)\right) ; \mathcal{P}\right) .
$$

5.6. Fiber products, Fubini symbols and Shannon expansion inequalities. Call (typically, surjective) measurable maps $X \rightarrow Y$ partitions of $X$ over $Y$ with the slices in $X$ thought of as the fibers. Given two spaces $X_{1}$ and $X_{2}$ partitioned over $Y$, denote by $X_{1} \times_{/ Y} X_{2} \subset X_{1}$ their fiber product, that is, the set of the pairs $\left(x_{1}, x_{2}\right) \in X_{1} \times X_{2}$ that lie over the same point in $Y$.

The set $X_{1} \times_{/ Y} X_{2} \subset X_{1}$ carries a unique Fubini measure such that its natural projections to $X_{1}$ and $X_{2}$ are measurable and consistent with the Fubini measures in the slices.

The space $X_{1} \times_{/ Y} X_{2} \subset X_{1}$, comes along with two fiber maps: the first one is induced from $P_{1}$, i.e., is given by the map $\left(P_{1}, \mathrm{id}_{2}\right): X_{1} \times / Y X_{2} \rightarrow X_{1} \times X_{2}$, where all slices isomorphically go to slices of $P_{1}$, and the second one is similarly induced from $P_{2}$. 
Given a family of partitions of a space $X$, or, better, a small category $C$ of measure spaces $X_{i}$ (where we do not distinguish any $X$ at this point) and measurable maps $P_{i j}: X_{i} \rightarrow X_{j}$, we compose the above operations and thus generate a small category $\mathscr{F}=\mathscr{F}(\mathcal{C})$ of measurable spaces and maps that is built inductively, starting from $\mathcal{C}$ by taking fiber products for new objects and the fiber maps for the morphisms.

We always include into this category the identity map of each space, corresponding to the partition into points as well the constant map, where the only slice is the space itself.

Examples. (1) If there is a single partition $P_{1}: X \rightarrow X_{1}$ of $X$ then all one essentially can do is to take the iterated graph of $P_{1}$ denoted $X^{k} / P_{1}=X^{k} / X_{1}$, that is, the set of points in $X^{k}$ that go to the same slice in $X$ under the $k$ projections $X^{k} \rightarrow X$. This $X^{k} / X_{1}$ comes along with $k$ (isomorphic but different) partitions, each of them is induced from $P_{1}$.

(2) Given a sequence of partitions $P_{i}, i=1,2, \ldots, k$, of $X$, define the space $Z(k)$ of $k$-chains of points $x_{0}, x_{2}, x_{2}, \ldots, x_{k}$, where every two consecutive points $x_{i-1}, x_{i}$ lie in some slice of $P_{i}$. This space is constructed by taking the consecutive fiber products of the graphs $X \times_{P_{i}} X$ over $X$ and thus it carries the Fubini measure on it. This $Z(k)$ is mapped to $X \times X$ by sending the two end points of chains of points back to $X$, but this map is not necessarily measurable.

For example, if $X=\Gamma$ is a locally compact group and $P_{i}$ are partitions into the orbits of closed subgroups $\Gamma_{i} \subset \Gamma$, then this map is measurable if the group product map $\times_{i} \Gamma_{i} \rightarrow \Gamma$ is open and countable-to-one.

The fiber product is neither a commutative nor an associative operation: a subset $Y \subset \Pi=\times_{i} X_{i}^{k_{i}}$ can be decomposed into a fiber product in many different ways (if at all). However,

the measures on $Y$ coming from these decompositions are all equal by the Fubini theorem and can be described with the following

Fubini symbols. Let $\mathcal{A}=\mathcal{A}(\mathcal{F})=\mathcal{A}(\mathcal{C})$ be the (Grothendieck) Abelian group generated by the symbols $[X]$ for all objects $X$ in $\mathscr{F}$ with the relations

$$
\left[X_{1} \times_{X_{3}} X_{2}\right]=\left[X_{1}\right]+\left[X_{2}\right]-\left[X_{3}\right]
$$

for all fiber products in $\mathcal{F}$. Clearly, $\mathcal{A}$ is generated by (the symbols of) the objects in $\mathcal{F}$.

Example. The space $Z$ of the $k$-chains of partitions of $X$ over $X_{i}$ has $[Z]=[X]+$ $\sum_{i}\left([X]-\left[X_{i}\right]\right)$. In particular, the $k$-iterated graph $Z$ of $X$ over $Y$ has $[Z]=$ $[X]+k([X]-[Y])$. 
Expansion coefficients of coordinate maps. Consider two objects $Z_{1}$ and $Z_{2}$ in $\mathscr{F}$ realized by subsets in the Cartesian products of $X_{i}$, say in $\times_{i} X_{i}^{k_{i}}$ and $\times X_{i}^{l_{i}}$ where $l_{i} \leq k_{i}$, and assume that some coordinate projection $p: \times_{i} X_{i}^{k_{i}} \rightarrow \times X_{i}^{l_{i}}$ sends $Z_{1}$ to $Z_{2}$.

Example. The above space of $k$-chains $c=\left\{x_{0}, x_{2}, x_{2}, \ldots, x_{k}\right\}$ in $X$ goes thus to $X \times X$ by $c \mapsto\left(x_{0}, x_{k}\right)$.

In general, such coordinate maps $p$ between $Z$ 's are not measurable and the pushforward $\lambda_{*}$ of the background measure $\lambda_{Z_{1}}$ from $Z_{1}$ to $Z_{2}$ may be everywhere infinite even for measurable $p$.

We are concerned with the case when $\lambda_{*}$ is locally finite, that is, $\lambda_{*}=\delta\left(z_{2}\right) \lambda_{Z_{2}}$ for a measurable function $\delta$ on $Z_{2}$ and we say in this case that $p$ is $\Delta$-expanding for a positive constant $\Delta$ if $\delta\left(z_{2}\right) \leq \Delta^{-1}$ (almost) everywhere on $Z_{2}$. Whenever such a map exists, we write the corresponding

log-inequality. $\left[Z_{2}\right] \geq\left[Z_{1}\right]+\log (\Delta)$.

Examples. (a) A map between countable spaces with unitary atoms is expanding, i.e., 1-expanding, if and only if it is injective.

(b) An injective smooth equidimensional map between Euclidean spaces is $\Delta$ expanding if its Jacobian $\geq \Delta$.

Shannon expansion theorem. Let $\mathcal{C}$ be a small category of Borel measure spaces with the maximal object $X$, where all other objects $X_{i}, i \in I$, are quotients $X / P_{i}$ for partitions $P_{i}$ of $X$. Let $p: Z_{1} \rightarrow Z_{2}$ be a $\Delta_{p}$-expanding map in the fiber-product category $\mathcal{F}$ generated by $\mathcal{C}$ and write the corresponding log-inequality in the symbols $[X]$ and $\left[X_{i}\right]$ (that generate the group $\mathcal{A}$ ),

$$
k[X]+\sum_{i} k_{i}\left[X_{i}\right] \geq l_{p}
$$

for the (positive and/or negative) integers $k$ and $k_{i}$ defined by (the Fubini symbols of ) $\left(Z_{1}, Z_{2}\right)$ and $l_{p}=\log \Delta_{p}$. Then every measure $\mu$ on $X$ satisfies

$$
k \cdot \operatorname{ent}(\mu)+\sum_{i} k_{i} \cdot \operatorname{ent}\left(\mu_{i}\right) \geq l_{p}
$$

for the push-forward (reduction) measures $\mu_{i}=\mu / P_{i}$ on $X_{i}$.

Proof. The claim is obvious if $\mu$ equals the restriction of $\lambda$ to a Borel subset $Y \subset X$ with $\lambda(Y)<\infty$ and such that the $P_{i}$-slices of $Y$ have constant Fubini measure for every partition $P_{i}$, since the Fubini measures are multiplicative for fiber products. Then, as before, the general case follows by the Gibbs tensorisation argument. 
5.7. Weighted Loomis-Whitney-Shearer and Brascamp-Lieb inequalities. Each $(\mathrm{ExSh})_{p}$ is a linear inequality in the variables $e$ and $e_{i}$ representing the entropies, where the Shannon expansion theorem says that

the closed convex hull $H_{\mathrm{ent}}=H_{\mathrm{ent}}\left(X, P_{i}\right)$ of the set of the vectors $\left(\operatorname{ent}(\mu)\right.$, ent $\left.\left(\mu_{i}\right)\right)$ in the Euclidean space $E_{+}=\mathbb{R}^{|I|+1}$ for $\mu$ running over all measures $\mu$ is contained in the intersection $H_{\text {ex }}$ of the closed half spaces defined by all (ExSh) $p$. Moreover, since the expansion condition passes to the Cartesian powers $\left(X^{N}, P_{i}^{N}\right)$, the (stabilized and possibly enlarged) closed convex hull $H_{\mathrm{ent}}^{\mathrm{st}} \subset \mathbb{R}^{|I|+1}$ of the vectors $\frac{1}{N}\left(\operatorname{ent}(\mu)\right.$, ent $\left.\left(\mu / P_{i}^{N}\right)\right)$ for all measures $\mu$ on $X^{N}$ is also contained in $H_{\mathrm{ex}}$.

Observe that the convex subsets $H_{\text {ent }}^{\text {st }} \subset H_{\mathrm{ex}} \subset \mathbb{R}^{|I|+1}$ are unbounded and only in rare cases $H_{\mathrm{ent}}^{\text {st }} \subset H_{\mathrm{ex}}$. On the other hand, the two sets are often parallel at infinity: if a linear form is bounded from above on $H_{\mathrm{ent}}^{\mathrm{st}}$ then it is also bounded on $H_{\mathrm{ex}}$.

Here is the simplest (ExSh).

1. Shannon and Loomis-Whitney inequalities for expanding families of partitions. If the (two ends) map from the space $Z(k)$ of chains to $X \times X$ for partitions $P_{1}, \ldots P_{i}, \ldots, P_{k}$ (see Example 2, p. 552) is expanding, then $P_{i}$ satisfy the Shannon inequality

$$
\sum_{i} \operatorname{ent}\left(P_{i}\right) \leq \operatorname{ent}(\mu)
$$

for all measures $\mu$ on $X$ and, therefore, the $P_{i}$-projections of every $Y \subset X$ satisfy the Loomis-Whitney inequality

$$
\prod_{i} \frac{\lambda(Y)}{\lambda_{i}\left(P_{i}(Y)\right)} \geq \lambda(Y)
$$

This inequality is sharp, i.e., $H_{\mathrm{ent}}^{\mathrm{st}}=H_{\mathrm{ex}}$, provided that the spaces $X / P_{i}$ are not monoatomic.

Example. The "expanding" assumption is (obviously) satisfied for the orbit (coset) partitions of a simply connected nilpotent Lie group $X=\Gamma$ by the orbits (cosets) of connected subgroups $\Gamma_{i}$ if the Lie algebras of $\Gamma_{i}$ are linearly independent and span the Lie algebra of $\Gamma$, provided that the product map $\times \Gamma_{i} \rightarrow \Gamma$ has the Jacobian $=1$ at the origin and the same is true for unipotent algebraic groups over the locally compact groups.

But the issuing Shannon and Loomis-Whitney inequalities are non-sharp for noncommuting subgroups $\Gamma_{i}$ (i.e., $\leq$ can be replaced by $<$ ), where one expects significantly stronger inequalities (due to non-commutativity) for the measures that are "sufficiently uniformly spread" over $\Gamma$. 
2. Shannon and Shearer inequalities for weighted coordinate partitions. Let $P_{J}$ be the coordinate plane partitions of $X=X_{I}=\times_{i \in I} X_{i}$ into the slices that are the fibers of the projections $X \rightarrow X_{I \backslash J}=\times_{i \in I \backslash J} X_{i}, J \subset I$, where the background measures $\lambda_{J}$ on $X_{J}=\times_{i \in J} X_{i}$ are not assumed to be product measures but rather satisfy the following assumption.

The measures $\lambda_{J}$ on $X_{J}=X_{i \in J} X_{i}$ are greater than the $\lambda_{I}$-Fubini measures on the $P_{J}$-slices: the (bijective!) coordinate projection of every $P_{J}$-slice $S \subset X$ to $X_{J}$ is expanding with respect to the $\lambda_{I}$-Fubini measure on $S$ and $\lambda_{J}$ on $X_{J}$.

This condition implies that all bijective coordinate maps between the fiber products of $X_{J}$ are expanding and the proof follows by the inclusion-exclusion principle. Thus

the coordinate inequalities from 5.3 remain valid under the above expansion assumption.

In particular, one has

Shearer-Loomis-Whitney projection inequality. The measures of the projections $P_{J}(Y) \subset X_{J}$ satisfy

$$
\prod_{J \subset I}\left(\lambda_{J}(Y)\right)^{\alpha_{J}} \geq \lambda_{I}(Y)
$$

for all $Y \subset X$ and all partitions of unity $\alpha_{J}$.

3. Euclidean Brascamp-Lieb inequalities. Let $P_{i}, i=1,2, \ldots, n$, be partitions of $\mathbb{R}^{k}$ into parallel non-coordinate affine subspaces. Then

the entropies of $P_{i}$ with respect to an arbitrary probability measure $\mu$ on $\mathbb{R}^{k}$ satisfy the inequality

$$
\left(\sum_{i} k_{i} \cdot \operatorname{ent}\left(P_{i}\right)\right)-\operatorname{ent}(\mu) \geq l
$$

for some real constants $k_{i} \geq 0$ and $-\infty<l<\infty$ (depending on $P_{i}$ but not on $\mu$, where one may always normalize to $l=0$ by rescaling the background measure $\lambda$ be $\exp (-l)$ ) if and only if they satisfy this inequality (with these very $k_{i}$ and $l$ ) for all Gaussian measures $v$ on $\mathbb{R}^{k}$.

This is usually stated in the (equivalent) Hölder form (see [12] and references therein).

The Shannon expansion theorem is weaker in the Euclidean case: it only delivers qualitative form of these inequalities, saying, in effect, that

the intersections of $H_{\mathrm{ent}}^{\mathrm{st}}$ and $H_{\mathrm{ex}}$, with the (convex) subsete $=-1, e_{i} \geq 0 \subset \mathbb{R}^{k}$, are parallel at infinity.

Sketch of the proof. All fiber products are Euclidean spaces and the coordinate projections are all linear; those which are bijective are $\Delta$-expanding for various $\Delta$ and 
these deliver a set of (ExSh)-inequalities that are, by simple linear algebra, equivalent, up to $l$ 's, to the Brascamp-Lieb inequalities.

This also applies to families of partitions into orbits of locally compact Abelian groups and nilpotent (and locally compact unipotent) Lie groups by families of subgroups, but, as in the above Loomis-Whitney case, this is less satisfactory (apart from being non-sharp even in the Abelian case) since the effects of non-commutativity (potentially strengthening such inequalities) are neglected.

One can slightly improve such inequalities similarly by adding to our category maps $\Pi$ permuting the coordinates of product spaces, where the additional terms in the Shannon inequalities come from non-invariance of measures on (and/or subsets in) such products.

These $\Pi$ may generate infinite groups of transformations of such products that admit no finite invariants measures.

Example. Let $P_{i}, i=1,2,3$ be the partitions of $\mathbb{R}^{2}$ into the three families of parallel lines in general position. Then the group generated by the three involutions of $\mathbb{R}^{4}$ corresponding to the three splitting of $\mathbb{R}^{2}$ into the product of lines contains a free subgroup. (But the resulting improvement of the Shannon inequality, even in this simple case, remains unsharp unlike the corresponding Brascamp-Lieb inequality.)

Remark (Riemannian manifolds). The $\Delta$-expansion condition makes sense for the exponential maps for Riemannian manifolds $X$, where, for example, it is satisfied with $\Delta=1$ by the complete simply connected manifolds with the sectional curvatures $K \leq 0$.

The geometric counterpart to the Loomis-Whitney inequality is a bound on the volume $|Y|$ of a $Y \subset X$ by some function of the (Liouville) measure $|S|$ of the set $S$ of geodesics that meet $Y$ in $X$. If $Y$ is convex, i.e., it meets every geodesics over a (connected and simply connected) segment, then $|S|=|\partial(Y)|$ with a suitably normalized Liouville measure and $|S| \leq|\partial(Y)|$ in general.

Non-sharp bounds follow by the Fubini theorem (as in the Coulhon-Saloff-Coste case), but a sharp inequality is available only for $X=\mathbb{R}^{n}$ by Almgren's variational argument [1] that applies to Riemannian manifolds $X$ (say, complete with bounded geometry, for safety) such that

the integral mean curvature $M$ of the "exposed" locally convex part $E$ of the boundary of every $Y$ admits a lower bound $M \geq I_{X}^{\prime}(|\partial(Y)|)$,

where a point $y \in \partial(Y)$ is called exposed if the set $C_{y}$ of the geodesic rays $s$ that start at $y$ and meet $Y$ at some $y^{\prime} \neq y$ contains no pair $(s,-s)$, and where the lower bound on $M$ depends on the function $I^{\prime}$ - a kind of a derivative of the isoperimetric profile.

If $X=\mathbb{R}^{n}$, then such bound on $M$ is obtained by taking the convex hull $H$ of $Y$, where the total Gauss curvature of $\partial(H)$ (which is independent of $H \subset \mathbb{R}^{n}$ ) minorizes 
the (obviously "homogenized") mean curvature $M$, since the Gauss curvature of $\partial(H)$ vanishes outside $E=\partial(Y) \cap \partial(H)$. Thus,

among all subsets $Y \subset \mathbb{R}^{k}$ of given $k$-volume, the round balls minimize the (Liouville) measure of the set of straight lines that intersect $Y$.

(If $Y$ are convex, this follows from the ordinary isoperimetric inequality.)

5.8. Linearized Shearer-Loomis-Whitney inequalities. Let $X_{i}, i \in I$, be vector spaces over some field and denote by $X_{J}, J \subset I$, the tensor product of $X_{i}$ over $J$, i.e., $X_{J}=\bigotimes_{i \in J} X_{i}$. Define the $J$-reduction $Y_{J} \subset X_{J}$ of a linear subspace $Y_{I} \subset X_{I}$ as the minimal subspace in $X_{J}$ such that $Y_{J} \otimes X_{I \backslash J}$ contains $Y_{I}$.

In order to recognize this better, take the tensor product $Z_{I}=X_{I} \otimes Y^{\prime}$ for some linear space $Y^{\prime}$, take a vector $z=Z_{I}$, and let $z_{J}: X_{I \backslash J}^{\prime} \otimes Y^{\prime} \rightarrow X_{J}$ be the homomorphism corresponding to $z$ under the canonical isomorphism $X_{I} \otimes Y^{\prime}=$ $\operatorname{Hom}\left(X_{I \backslash J}^{\prime} \otimes Y^{\prime}, X_{J}\right)$, where $X_{I \backslash J}^{\prime}$ denotes the linear dual of $X_{I \backslash J}$.

Then the $J$-reduction of the image $Y \subset X_{I}$ of $z_{I}$ equals the image of $z_{J}$ in $X_{J}$; thus, $\operatorname{rank}\left(Y_{J}\right)=\operatorname{rank}\left(z_{J}\right)$.

Tensorial reduction inequality. The ranks of the $J$-reductions of every linear subspace $Y \subset X_{I}=\bigotimes_{i \in I} X_{i}$ satisfy

$$
\prod_{J \subset I}\left(\operatorname{rank}\left(Y_{J}\right)\right)^{\alpha_{i}} \geq \operatorname{rank}(Y)
$$

for an arbitrary partition of unity $\left\{\alpha_{J}\right\}$ on I.

Proof. Take bases $B_{i}$ in each $X_{i}$, let $B_{I}=\bigotimes_{i \in I} B_{i}$ be the corresponding basis in $X_{I}$ and project $Y$ to a coordinate plane $P$ along the complementary coordinate plane. Clearly, $|J|_{T} \leq|J|_{Y}$ for all $Y \subset X_{I}$ and all $J \subset I$. Since there is a $P$ such that the coordinate projection $Y \rightarrow P$ is bijective, the above $\left(\mathrm{P}_{\circ}\right)$ applies and yields $\left(\mathrm{P}_{\otimes}\right)$.

Remark. Given a $(k+1)$-linear form $y=y\left(x_{0}, \ldots, x_{k}\right)$, each splitting $J$ of the variables $x_{i}$ into two groups defines a bilinear form $y_{J}$ (on the tensor product corresponding to the splitting) and $\left(\mathrm{P}_{\otimes}\right)$ becomes an inequality between the ranks of the forms $y_{J}$.

A particular instance of this (where one may have stronger inequalities) is the cohomology (sub)algebra $X$ of an (algebraic) manifold $V$, where $y\left(x_{i}\right)$ equals the value of the cup-product of $x_{i}$ on the fundamental class of $V$. (See [16] for topological applications of such inequalities.)

5.9. Orbit and Lieb-Ruskai inequalities for representations of compact groups. Consider a finite dimensional linear representation $Z$ of a group $\Gamma$ with a given family of subgroups $\Gamma_{J}, J \in \mathcal{g}$, take a vector $z \in Z$, and let $\left|\Gamma_{J}(z)\right|$ denote the rank of linear span of the $\Gamma_{J}$-orbit of $z$. 
If $\Gamma$ is the Cartesian product, $\Gamma=\times_{i} \Gamma_{i}, i \in I$, and $\Gamma_{J}=\times_{i \in J} \Gamma_{i}$ we have the following

Linearized Shearer inequality. If the representations of $\Gamma$ and all $\Gamma_{J}$ are semisimple (over the algebraic closure of the ground field), then, for an arbitrary partition of unity $\left\{\alpha_{J}\right\}$ on $I$, the rank of the linear span of the $\Gamma$-orbit of every point $z \in Z$ satisfies

$$
|\Gamma(z)| \leq \prod_{J \subset I}\left|\Gamma_{J}(z)\right|^{\alpha_{J}} .
$$

Proof. If $Z$ is a multiple of an irreducible representation, i.e., $Z=\left(\bigotimes_{i} X_{i}\right) \otimes Y^{\prime}$ for irreducible representations $X_{i}$ of $\Gamma_{i}$, then the linear span of the $\Gamma_{J}$ orbit of $z$ equals the tensor product of $\bigotimes_{i \in J} X_{i}$, with the image of the corresponding homomorphism $z_{J}^{\prime}:\left(\bigotimes_{i \in J} X_{i}\right)^{\prime} \rightarrow\left(\bigotimes_{i \in I \backslash J} X_{i}\right) \otimes Y^{\prime}$, and the above tensorial inequality applies.

Then the general case follows by applying $\left(\mathrm{P}_{\circ}\right)$ from 5.7 to the Cartesian product of the sets $R_{i}$ (earlier denoted $X_{i}$ ) of irreducible representations of $\Gamma_{i}$ with the measures assigning to each representation its rank weighted on $R_{J}$ by the multiplicity with which the irreducible representation $r_{J}$ of $\Gamma_{J}$ enters the span of the orbit $\Gamma_{J}(z)$.

Remarks. (a) The above applies to the representations of finite groups over the fields of the characteristic prime to $|\Gamma|$ and also to the real and complex representations of compact groups; it is unclear whether the semisimplicity assumptions can be removed or relaxed.

(b) The inequality $\left(\mathrm{P}_{*}\right)$ over $\mathbb{C}$ admits an entropic (and, probably, a Hölder) refinement with the Lieb-Ruskai inequality for quantum entropy. The quantum (von Neumann) entropy is defined for positive self-adjoint operators with unit traces on, say, finite dimensional Hilbert spaces $Z$, or, equivalently, for positive (semi definite) quadratic forms $\mu$ on $Z$, similarly to the Boltzmann entropy.

A quadratic form $\mu$ is regarded as a function on the linear subspaces in $Z$ that assigns to each $Y \subset Z$ the trace of $\mu$ restricted to $Y$, where this function is additive under the spans of mutually orthogonal subspaces. If $P_{Y}$ is the orthogonal projection onto a subspace $Y \subset Z$ and $\mu=\operatorname{dim}(Y)^{-1} P_{Y}$, then, by definition, ent $(\mu)=$ $\log (\operatorname{dim}(Y))$.

Then, in general, one passes to the tensorial powers $\left(Z^{\otimes N}, \mu^{\otimes N}\right)$, takes the $\varepsilon$ approximation (in the trace norm) of $\mu^{\otimes N}$ by a "measure" $\mu_{\varepsilon}^{N}$ associated to a projection on a subspace $Y_{N} \subset Z^{\otimes N}$ of maximal (depending on $\varepsilon$ dimension), and set

$$
\operatorname{ent}(\mu):=\lim _{\substack{N \rightarrow \infty \\ \varepsilon \rightarrow 0}} \frac{1}{N} \operatorname{ent}\left(\mu_{\varepsilon}^{N}\right) .
$$

The law of large numbers (for the spectral measure of $\mu$ ) shows that ent $(\mu)$ equals the entropy of the Boltzmann entropy of the spectral measure, where the background 
measure (corresponding to the background Hilbert structure on $Z$ ) is that of a discrete space with all atoms of unit weight.

Given such a "measure" on a tensor product of Hilbert spaces, $L=L_{1} \otimes L_{2}$, one defines its reductions $\mu_{i}$ on $L_{i}, i=1,2$, say $\mu_{1}$, by taking the traces of the corresponding self-adjoint operators over $L_{2}$. Equivalently, one can average $\mu$ over a compact group $\Gamma_{2}$ unitarily and irreducibly acting on $L_{2}$, and thus, on $L$, where the averaged "measure", say $\mu_{1}^{\prime}$ on $L$, can be uniquely written as $\mu_{1}^{\prime}=\mu_{1} \otimes \lambda_{2}$ for $\lambda_{2}$ corresponding to the scalar operator on $L_{2}$ with trace $=1$ that is $\left|L_{2}\right|^{-1}$. id.

Lieb-Ruskai strong superadditivity. Let $\mu$ correspond to a positive self-adjoint operator with trace $(\mu)=1$ ("probability measure") on the tensor product of three finite dimensional Hilbert spaces, $L=L_{1} \otimes L_{2} \otimes L_{3}$. Then the entropies of its reductions to $L_{1} \otimes L_{2}, L_{2} \otimes L_{3}$ and $L_{2}$ satisfy

$$
\operatorname{ent}\left(\mu_{12}\right)+\operatorname{ent}\left(\mu_{23}\right) \geq \operatorname{ent}\left(\mu_{2}\right)+\operatorname{ent}\left(\mu=\mu_{123}\right) .
$$

(See [26], [5] and references therein.)

This inequality (trivially, by the inclusion-exclusion principle) implies the (weighted with partitions of unity) Shannon type inequalities for tensor products of (more than three) Hilbert spaces and thus for Hilbert spaces acted upon by Cartesian products of compact groups. This sharpens the above reduction inequalities when the underlying field $\mathbb{F}=\mathbb{C}$. Furthermore, this generalizes to the actions of infinite groups $\Gamma_{i}$ on $l_{2}\left(\Gamma=\times_{i} \Gamma_{i}\right)$, where the entropy is defined via the von Neumann $\Gamma_{i}$-dimensions ([23]). But this does not seem (at least, at the first glance) to yield Loomis-Whitney projection (reduction) inequalities (nor isoperimetric inequalities) for finite dimensional subspaces $D \subset l_{2}(\Gamma)$ for non-Abelian $\Gamma_{i}$.

Questions. (a) The quantum entropy is a concave function on the space $M_{n}$ of positive selfadjoint operators on $\mathbb{R}^{n}$ (by the superadditivity of the quantum entropy proved by Landford prior to the Lieb-Ruskai theorem) and the geometry of the corresponding "quantum" (Fisher-)Kubo-Mori-Bogolubov metric and similar Bures-Uhlmann and Hasegawa-Nagaoka-Pets metrics (see [24]) along with the symmetric Riemannian metric on $M_{n}=\mathrm{GL}_{n} / \mathrm{O}_{n}$, probably, tell you something else about (relations between the quantum entropies of) various reductions. Furthermore, the relative entropy defined on the pairs of positive selfadjoint operators by $\operatorname{trace}(\mu \log (\mu)-\mu \log (v))$ is also concave by the Lieb-Ruskai theorem which expresses the positivity of the metric given by the Hessian of this trace. (See [21] for the computation of curvatures of such metrics.)

Is there a "natural" (symplectic/Kählerian?) definition of these metrics that reveals their geometry and makes transparent the entropy inequalities?

(b) Do the Euclidean Brascamp-Lieb inequalities remain valid for the quantum entropy of operators (say, with continuous compactly supported kernels $K\left(x_{1}, x_{2}\right)$ ) 
on the $L_{2}$-Hilbert space $L_{2}\left(\mathbb{R}^{n}, d x\right)$ (for the Lebesgue and/or for the Gauss measure $d x$ on $\mathbb{R}^{n}$ ) with respect to the reductions in the tensorial decompositions $L_{2}\left(\mathbb{R}^{n}\right)=$ $L_{2}\left(X_{1}\right) \otimes L_{2}\left(X_{2}\right)$ for the (not necessarily coordinate) orthogonal decompositions $\mathbb{R}^{n}=X_{1} \oplus X_{2}$ ?

5.10. Reduction inequality for orderable groups $\Gamma$. Let $\Gamma=\times_{i} \Gamma_{i}, i \in I$, take a linear space $B$ of $\mathbb{F}$-functions on $\Gamma$ with finite supports for some field $\mathbb{F}$ and recall the asymptotic $\Gamma_{J} \backslash$-dimensions $\left|D: \Gamma_{J}\right|$ (see 1.10) for $\Gamma_{J \backslash}=\times_{i \in I \backslash J} \Gamma_{i}$. By combining the Shearer-Loomis-Whitney projection inequality $\left(\mathrm{P}_{\circ}\right)$ with the equivalence $\circ \Longleftrightarrow *$ with order (see 3.2) we conclude that

if $\Gamma$ is a left orderable amenable group, then

$$
\operatorname{rank}(B) \leq \prod_{J \subset I}\left|D: \Gamma_{J}\right|^{\alpha_{J}}
$$

for all partitions of unity $\left\{\alpha_{J}\right\}$ on I and all linear (sub)spaces $B$ of functions on $\Gamma$ with finite supports.

Furthermore, if the product map $\times_{i} \Gamma_{i} \rightarrow \Gamma$ for amenable subgroups in an orderable (not necessarily amenable) $\Gamma$ is one-to-one, then

$$
(\operatorname{rank}(D))^{k-1} \leq \prod_{i=1,2, \ldots, k}\left|D: \Gamma_{i}\right|
$$

for all linear spaces $D$ of functions on $\Gamma$ with finite supports.

Remarks. (a) The above generalizes to other types of "expanding" families of subgroups, e.g., in Abelian $\Gamma$ with the use of the corresponding Brascamp-Lieb kind of inequalities. These, however, are not so good as far as the Brascamp-Lieb constant $l$ is concerned, albeit the discrete version, delivered by the Shannon expansion inequality, is sharp for the trivial reason: the presence of the $\delta$-measures. A true generalization/linearization of the Brascamp-Lieb inequality for (discrete) groups $\Gamma$ and their group algebras $A$ needs additional invariants of measures in $\Gamma$ and/or linear subspaces in $A$ that would rule out their concentration on small subsets.

(b) If $\Gamma=\mathbb{Z}^{I}$, then $\left(\mathrm{P}_{*}\right)$ applies to linear subspaces $D$ in the algebra of regular functions on Zariski open subsets in $\mathbb{F}^{I}$, where the $J$-reduction of $D$ becomes the restriction $D_{J}$ of (the functions in) $D$ to a generic $J$-plane in $\mathbb{F}^{k}$ with $\left|D_{J}\right|=\left|D: \Gamma_{J}\right|$. The so interpreted $\left(\mathrm{P}_{*}\right)$ makes sense and remains valid for the algebra of formal (and convergent, for normed fields) power series at a point in $\mathbb{F}^{I}$. Furthermore, there are similar inequalities for the restrictions of $D$ to generic members of non-plane families of algebraic (analytic) subvarieties in $\mathbb{F}^{I}$.

5.11. -convexity and the entropic Følner functions $\boldsymbol{F}_{\text {.. }}$ A positive monotone increasing function $F(n), n>n_{0}>0$, is called •-convex if $\log F\left(\frac{1}{\varepsilon}\right), 0<\varepsilon \leq 1 / n_{0}$, is convex, that is equivalent to the convexity of $J(\exp (l))$, where $\frac{1}{J}$ is the inverse function of $F$. 
Examples. The functions const $\cdot n^{k}$ are $\bullet$-convex for all $k \geq 1$ and const $>0$. Also the functions const $\cdot \exp \left(n^{a}\right)$ are $\bullet$-convex for $a \geq 0$.

Every positive monotone increasing function $F(n)$ admits a unique maximal •convex minorant $F_{\mathbf{0}}(n) \leq F(n)$ and this $F_{\bullet}$, as it is easy to see, has growth (roughly) comparable to that of $F$,

$$
F .(n) \geq \min _{\alpha} \max \left(\alpha F(n), F(n)^{1-\alpha}\right)
$$

for $0 \leq \alpha \leq 1$.

Given a positive function ("isoperimetric profile") $I(r), r \geq 0$, define the associated entropic $F \varnothing$ lner function, denoted $F$. $(n)$ as the above maximal $\bullet$-convex minorant of the ordinary Følner function $F(n)=F_{I}(n)$. Clearly, the inverse function to $F$. equals the minimal monotone increasing majorant $M(r)$ of $r / I(r)$ for which $\frac{1}{M(\exp (l))}$ is convex.

If $X$ is a $\Gamma$-space with the orbit partition $P$ and the ordinary Følner function $F$, then $F$. minorizes (not only the cardinalities of the subsets $Y \subset X$ with $|\partial(Y)| \leq n$ but also) the entropy of the partition $P \mid Y$, of $Y$ :

If $|\partial(Y)| /|Y| \leq 1 / n$, then

$$
\operatorname{ent}(P \mid Y):=|Y|^{-1} \sum_{s \in P \mid Y} r_{s} \log \left(r_{s}\right) \geq \log \left(F_{\bullet}(n)\right)
$$

for $r_{s}=|s \cap Y|$, where the sum is taken over the $P$-slices $s$ of $P \mid Y$.

Proof. The boundary of $Y$ is bounded from below, according to the definition of $I(r)$, by

$$
|\partial Y| \geq \sum_{s} I\left(r_{s}\right) /|Y|=\sum_{s} \frac{r_{s}}{\sum_{s} r_{s}} J\left(r_{s}\right)
$$

for $J(r)=I(r) / r$. If $J(\exp (l))$ is convex, then

$$
\sum_{s} \frac{r_{s}}{\sum_{s} r_{s}} J\left(r_{s}\right) \geq J\left(\exp \frac{\sum_{s} r_{s} \log \left(r_{s}\right)}{\sum_{s} r_{s}}\right)=J(\exp (\operatorname{ent}(P \mid Y))) .
$$

The claim follows from the definition of the Følner function $F=F_{\circ}$ of the action.

The above equally applies to general partitions $P$ (with $\sum_{s} I\left(r_{s}\right) /|Y|$ taken for the definition of the "boundary") and shows that the Følner transform of •-convex functions for families of partitions $P_{i}$ of an $X$ is governed by the lower bounds on the entropy of subsets $Y$ (recall that $\operatorname{ent}(Y):=\log |Y|$ for discrete spaces) by the entropies of the partitions. In particular, if

$$
\operatorname{ent}(Y) \geq \sum_{i} \kappa_{i} \operatorname{ent}\left(P_{i} \mid Y\right)+\log (\beta)
$$


for all $Y \subset X$, then the Følner transform $F=F\left(n_{i}\right)$ of $\bullet$-convex functions $F_{i}$ satisfies

$$
F\left(n_{i}\right) \geq \beta F_{i}\left(n_{i}\right)^{\kappa_{i}} .
$$

Thus, for example,

the Shannon inequalities yield (often sharp) lower bounds on the multivariable entropic Folner functions of Cartesian products $X=\times_{i} X_{i}$ of $\Gamma_{i}$-spaces in terms of the entropic Folner functions of individual $X_{i}$ and/or of $X_{J}=\times_{i \in J} X_{i}$, $J \subset I$.

This applies to the ordinary Følner functions if these are --convex or (well) approximated by $\bullet$-convex functions. The simplest instance of this is the (multivariable) isoperimetric inequalities for free Abelian groups where the (ordinary) Følner functions are $\bullet$-convex.

Similarly,

the Shannon inequalities provide lower bounds on the Folner functions of discrete groups $\Gamma$ with families of subgroups $\Gamma_{i}, i=1,2, \ldots, k$, where the product map $\mathrm{X}_{i} \Gamma_{i} \rightarrow \Gamma$ is injective and

the Brascamp-Lieb inequalities give similar bounds on the multivariable Følner function of $\Gamma=\mathbb{R}^{k}$ with a given family of linear subspaces $\Gamma_{i} \subset \Gamma$, where one may use the Folner functions $F_{i}$ defined with the ordinary Euclidean boundaries of subsets in $\Gamma_{i}$.

By combining the above with the Schwartz symmetrization for normal extensions,

$$
1 \longrightarrow \Gamma_{1} \longrightarrow \Gamma \longrightarrow \Gamma_{2} \longrightarrow 1
$$

we conclude that

the entropic Folner function of $\Gamma$ is bounded from below by the product of these of $\Gamma_{i}, i=1,2$.

Finally, we recall that $\circ \Longleftrightarrow *$ (see 3.2) and obtain a similar inequality for the linear algebraic $F_{*}(\Gamma)$ for the space of functions with finite supports on $\Gamma$ :

If the group $\Gamma_{2}$ is orderable and the Folner functions $F_{*}\left(n_{1}, \Gamma_{1}\right)$ and $F_{\circ}\left(n_{2}, \Gamma_{2}\right)$ are minorized by $\bullet$-convex functions $F_{i}\left(n_{i}\right), i=1,2$, then

$$
F_{*}\left(n_{1}, n_{2} ; \Gamma\right) \geq F_{1} \cdot F_{2} .
$$

5.12. Entropic Poincaré inequalities. Consider a measure $\mu=f(x) \lambda$ on a $\Gamma$ space $X$, where $\lambda$ is an invariant measure on $X$, e.g., $X=\Gamma$ with the Haar measure, and let $Y_{\mu}=\{f(x) \leq m\} \subset X \times \mathbb{R}_{+}$be the subgraph of $f$.

We define $|\partial(\mu)|=\left|\partial\left(Y_{\mu}\right)\right|$ for the obvious action of $\Gamma$ on $X \times \mathbb{R}_{+}$(for whichever notion of the boundary for subsets) and then apply the above entropic inequality to the partition of $Y_{\mu}$ to the levels $f(x)=m, m \in \mathbb{R}_{+}$. Since the entropy of this partition is $\leq \operatorname{ent}(\mu)$ by the Shannon inequality, we see that 
if the Følner function of $X$ is minorized by $a \cdot$-convex function $F(n)$, then

$$
\operatorname{ent}(\mu) \geq F(|\mu| /|\partial(\mu)|)
$$

for all finite measures $\mu$ on $X$ (where, recall that $|\mu|:=\mu(X)=\left|Y_{\mu}\right|$ and $\operatorname{ent}(\mu)=\log |\mu|-|\mu|^{-1} \int_{S} \log \frac{1}{f} d x$ for the support $S \subset X$ of $\left.\mu\right)$.

Remarks. (a) The above log-Poincaré (Sobolev) inequality in the $\mathbb{R}^{k}$ case holds, in the sharp form, for the Euclidean boundary (see [12]).

(b) Measures $\mu$ on $X$, rather than subsets $Y \subset X$, make a natural domain of definition for the Følner transform and the entropic Følner functions. In fact, everything in the previous section (trivially) generalizes to measures, where some of the resulting log-Poincaré inequalities become sharp in this context. For example, the extremal measures for the log-Poincaré inequalities associated to the Euclidean Brascamp-Lieb inequalities are the Gaussian ones. (See [12] and references therein.)

\section{Lower bounds on $F_{*}$ for groups with large orderable subgroups}

We bound from below the linear algebraic Følner functions of groups $\Gamma$ by applying the projection inequalities to certain infinitely generated orderable (e.g., free Abelian) subgroups in $\Gamma$.

6.1. Expanding subgroups inequality. Let $\Gamma$ be a group with a finite generating subset $G \subset \Gamma$, and let $N=N(n)$ denote the maximal number of elements $g_{i} \subset$ $G^{\cdot n} \subset \Gamma$ such that

(a) the subgroup $\Gamma^{N}, N=N(n)$, generated by $g_{i}, i=1,2, \ldots, N$, is orderable;

(b) the product map of the (infinite cyclic) subgroups $\Gamma_{i}$ generated by $g_{i}$ to $\Gamma$, that is, $\mathrm{X}_{i=1,2, \ldots, N(n)} \Gamma_{i} \rightarrow \Gamma$ is injective.

Then

$$
F_{*}(n ; \Gamma, G) \geq \frac{1}{2} 2^{N\left(\frac{n}{2}\right)} .
$$

Proof. Let $\mu$ be the probability measure uniformly distributed over $g_{1}, \ldots, g_{N(n)}$. Then the Loomis-Whitney inequality for expanding partitions implies that all finite subsets $Y \subset \Gamma^{N}$ with

$$
\left|\partial_{\mu}\right|(Y) \leq \frac{1}{2}|Y|
$$

have $|Y| \geq 2^{\frac{n}{2}}$. The claim follows with $G^{* m}$ (see 1.4 and 1.6). 
This can be modified and generalized in a variety of directions. For example, there is a similar inequality for groups $\Gamma$, where some orderable subgroups $\Gamma^{N} \subset \Gamma$ admit $N$-long normal series, where the linearized isoperimetry is conveniently done with $F$.-functions.

However, these inequalities do not give much new compared to what follows by other means.

6.2. Distorted and undistorted actions on $l_{2}$ and on decaying $p$-adic functions. Consider an action of the group $\Gamma=\mathbb{Z}^{k}$ on a linear space $L$ over a field $\mathbb{F}$ and show that the following properties of such an action are equivalent:

(1) The action is free: if $\sum_{\gamma} c_{\gamma}(l)=0$, then either all $c_{\gamma} \in \mathbb{F}$ or $l \in L$ are zero.

(2) The action is non-degenerate: $|D: \Gamma|>0$ for all non-zero subspaces $D \subset L$, i.e., if $H_{i} \subset \Gamma$ is an exhaustion of $\Gamma$ by Følner sets then

$$
\limsup _{i \rightarrow \infty}\left|H_{i} * D\right| /\left|H_{i}\right|>0 .
$$

(3) The action is uniformly non-degenerate:

$$
\inf _{D} \liminf _{i \rightarrow \infty}\left|H_{i} * D\right| /\left|H_{i}\right|>0 .
$$

(4) The spans of these $H_{i}$-orbits satisfy

$$
\limsup _{i \rightarrow \infty}\left|H_{i} * D\right| /\left|H_{i}\right|^{\frac{k-1}{k}}=\infty .
$$

(5) The action of the group $\mathbb{F}$-algebra $A$ of $\Gamma$ on $L$ does not factor through an algebra of transcendence degree $<k$.

To see the equivalence of these conditions, observe that every finitely generated module $L$ over $A$ equals the set of $\mathbb{F}$-sections of a coherent sheaf $\mathscr{L}$ over the torus $\left(\overline{\mathbb{F}}^{\times}\right)^{k}$, where obviously (1)-(5) are satisfied if and only if $\mathscr{L}$ is not supported on a proper subvariety in $\left(\overline{\mathbb{F}}_{\times}\right)^{k}$.

Remark. It is clear that (1) $\Longrightarrow(3) \Longrightarrow$ (2) for all amenable groups $\Gamma$; furthermore, if the group algebra $A$ of $\Gamma$ contains no non-constant finite dimensional subalgebras (e.g., $A$ has no zero divisors) then also (2) $\Longrightarrow(1)$; but it is unclear what corresponds to (4) and (5) in the general case.

Examples: Functions with fast decay. (a) The action of $\Gamma=\mathbb{Z}^{k}$ on the Hilbert space $l_{2}(\Gamma)$ satisfies the conditions (1)-(5), and the same remains true for the space $l_{2}(X)$, where $X$ is a set with a free action of $\Gamma$ by the following theorem of Gabor Elek (see [7]).

If the group algebra of an amenable group $\Gamma$ over $\mathbb{R}$ has no zero divisors, then the action of $\Gamma$ on $l_{2}(\Gamma)$ is free. 
Alternative proof. Regard $\mathbb{C}$-valued (moderately growing) functions $a$ on $\mathbb{Z}^{k}$ as Fourier transforms of distributions $\hat{a}$ on the torus $\mathbb{T}^{k}$ : if this distribution has a Zariski dense support, then the linear span $L$ of $a$ satisfies (1)-(5), as we have just seen. In particular, this proves (1)-(5) for $l_{2}\left(\mathbb{Z}^{k}\right)$ since the $\hat{a} \in L_{2}\left(\mathbb{T}^{k}\right)$ for $a \in l_{2}\left(\mathbb{Z}^{k}\right.$ and, being a measurable function, $\hat{a}$ has Zariski dense support.

(b) The spaces of decaying functions on $\mathbb{Z}^{k}$ with values in discrete valuation fields also satisfy (1)-(5) since they are representable by power series.

(c) Counterexample for decaying $\mathbb{C}$-valued functions. The Fourier transform $a$ of a distribution $\hat{a}$ supported on an algebraic subvariety $V \subset \mathbb{T}^{k}$ of positive codimension is annihilated by a polynomial in the group algebra of $\mathbb{Z}^{k}$ that vanishes on $V$ with a sufficient multiplicity; thus (a) is violated. But such an $a=a\left(n_{i}\right)$ may be a decaying function on $\mathbb{Z}^{k}$.

For instance, let $V \subset \mathbb{T}^{k}$ be a smooth subvariety in that is nowhere infinitesimally flat (if $V$ is analytic this means that it is not contained in a sub-torus of $\mathbb{T}^{k}$ ) and $\hat{a}$ be given by a smooth measure on $V$. Then $a$ does decay, being expressed by an oscillating integral on $V$.

The fastest rate of decay of such an $a$ is $O\left(\sum_{i=1,2, \ldots, k}\left|n_{i}\right|\right)^{\frac{1-n}{2}}:$ smooth hypersurfaces $V \subset \mathbb{T}^{k}$ with non-vanishing Gaussian curvature, e.g. round spheres, give such decay. (This was pointed out to me by Terence Tao.)

Question. Does a faster rate of decay, i.e., $o\left(\sum_{i=1,2, \ldots, k}\left|n_{i}\right|\right)^{\frac{1-n}{2}}$, rules out distributions with Zariski non-dense supports?

Domination lemma. Let $L$ be a space of functions on $\Gamma=\mathbb{Z}^{k}$ that is finitely generated as a $\mathbb{Z}^{k}$-module and satisfies (1)-(5). Then the isoperimetry of L dominates (as defined in 3.2) that of $\Gamma$. Consequently, the linear spaces of the real $l_{2}$-functions and the fast decaying $p$-adic functions on $\Gamma$ have the same isoperimetry as $\Gamma$ itself.

Proof. The linear space in question equivariantly embeds into the space of formal power series, and the domination by ordering from 3.2 applies.

In particular, it follows from the lemma that the combinatorial projection inequalities for families of partitions of $\mathbb{Z}^{k}$, such as the Loomis-Whitney and Shearer inequalities, pass to the linear subspaces $D$ in these spaces $L$ of functions, where the cardinality of the image of the projection $Y \rightarrow \mathbb{Z}^{k} / \Gamma_{i}$ for a (free Abelian) subgroup $\Gamma_{i} \subset \mathbb{Z}^{k}$ is replaced by the dimension of the span of the $\Gamma_{i}$-orbit of $D$ over the fraction field of the group ring of $\Gamma_{i}$.

Here are two applications.

1. Split abelian subgroup inequality. Let $\Gamma$ be a group with a finite generating subset $G \subset \Gamma$ and let $N=N(n)$ denote the maximal number of elements $g_{i} \subset G^{\cdot n} \subset \Gamma$ 
such that $g_{i}, i=1,2, \ldots, N(n)$, generate a free Abelian subgroup of rank $N(n)$. Then the max-Folner functions $F_{*}$ of $l_{2}(\Gamma)$ and of the spaces of decaying functions with values in ultrametric fields satisfy

$$
F_{*}(n ; \Gamma, G) \geq \frac{1}{2} 2^{N\left(\frac{n}{2}\right)} .
$$

Proof. Use the measure $\mu$ uniformly distributed on $g_{i}$ and proceed as in the above expanding subgroups inequality.

2. Distorted abelian subgroups inequality. Let $\Gamma$ contain a finitely generated free Abelian subgroup $\Gamma_{0}$ and let $G \subset \Gamma$ be a generating subset containing the generators of $\Gamma_{0}$. Then the max-Folner functions $F_{*}$ for the spaces of $l_{2}$-functions and of decaying "ultrametric" functions on $\Gamma$ are bounded from below by the relative growth function $G_{\circ \circ}(n)=\left|\Gamma_{0} \cap G^{\cdot n}\right|$ as follows,

$$
F_{*}(n ; \Gamma, G) \geq \frac{1}{2} G_{* *}\left(\frac{n}{2}\right) .
$$

Proof. Use the measure $\mu$ uniformly distributed on $\Gamma_{0} \cap G^{\circ n}$ and apply the linearized displacement inequality from with the $G^{* n}$-inequality as in the Coulhon-Saloff-Coste argument in 1.4.

Statements 1 and 2 can be applied, e.g., to solvable groups as these have many Abelian subgroups.

Example. Every non-virtually nilpotent polycyclic group contains an Abelian subgroup with exponentially growing $G_{\circ}$ ( this is well known and easy); therefore

the $l_{2}$ and "ultrametric" Folner functions $F_{*}(n)$ grow exponentially for all nonvirtually nilpotent polycyclic groups.

Questions. Can one replace "polycyclic" by "solvable without torsion" with a suitable class of $\mu$ generalizing those in 1 and 2 ?

Is the "free Abelian" requirement truly necessary in 1 and 2 or something like "orderable" would suffice?

Remark. The linearized L(oomis)-W(hitney)-S(hearer) inequality, that is, an upper bound on the dimension of a linear space $D$, say, of (germs of) analytic functions on $\mathbb{R}^{k}$ (or any other class of functions with similar genericity properties) by the dimensions of the restrictions of $D$ to generic members of parallel families of the coordinate planes, can be proven by induction on $k$ and $\operatorname{dim}(D)$ as follows.

Take a generic hyperplane $H \subset \mathbb{R}^{k}$ parallel to the coordinate hyperplane $\mathbb{R}^{k-1} \subset$ $\mathbb{R}^{k}$, let $D \mid H$ be the restriction of $D$ to $H$ and $D_{/ H} \subset D$ be the subspace of functions 
that vanish on $H$. Then the LWS inequalities for $D \mid H$ and $D_{/ H}$ yield that for $D$ by the Minkovski inequality $\left(\prod_{j}\left(a_{j}+b_{j}\right)\right)^{\frac{1}{l}} \geq\left(\prod_{j} a_{j}\right)^{\frac{1}{l}}+\left(\prod_{j} b_{j}\right)^{\frac{1}{l}}$. (There are similar inequalities for non-coordinate "webs of subvarieties" that we shall not discuss here.)

6.3. Isoperimetry with "large" $\boldsymbol{H} \subset \boldsymbol{\Gamma}$. The above suggest regarding (various) boundaries $|\partial|_{H}(Y)$ as something depending on the size of $H$ as well as of $Y$.

Here are two examples for $\Gamma=\mathbb{Z}$ and a subset $H \subset \mathbb{Z}$.

(1) Let $\mu$ be the probability measure uniformly distributed on $H$. Then all finite subsets $Y \subset \mathbb{Z}$ satisfy

$$
\left|\partial_{\mu}(Y)\right|:=\frac{1}{H} \sum_{h \in H}\left|\partial_{h}(Y)\right| \geq \min \left(\frac{1}{2}|Y|, \frac{1}{8}|H|\right) . \quad\left(\partial_{\mu} \succ \min \right)
$$

To show this, consider two cases.

Case 1 . Let $|H| \geq 2|Y|$. Then the claim follows from the displacement inequality in 1.4 .

Case 2. Divide $H$ into positive and negative parts, $H=H_{+} \cup H_{-}$, and let $Y_{-} \subset|Y|$ be the first (for the natural order) $k_{-}$elements in $Y$ for $k_{-}=\left|H_{-}\right|$and $Y_{+} \subset|Y|$ be the last $k_{+}=\left|H_{+}\right|$elements. Clearly,

$$
\left|\partial_{H_{+}}\left(Y_{+}\right)\right| \leq\left|\partial_{H_{+}}(Y)\right| \text { and }\left|\partial_{H_{-}}\left(Y_{-}\right)\right| \leq\left|\partial_{H_{-}}(Y)\right|
$$

and the proof reduces to Case 1 .

Remarks. (a) The inequality $\left(\partial_{\mu} \succ \min \right)$ yields a lower bound on the max-profile of $\mathbb{Z}$ with respect to $H$, but the exact evaluation of $I_{\circ}^{\max }(r ; \mathbb{Z}, H)$, even for $H=$ $\{1,2, \ldots, k\}$, is not apparent and seems to pertain to combinatorial number theory.

(b) The inequality $\left(\partial_{\mu} \succ \min \right)$ obviously remains true for all biorderable groups $\Gamma$.

(c) Let $H_{i} \subset \Gamma, i=1,2, \ldots k$, be subsets such that the product map $\left(h_{1}, h_{2}, \ldots, h_{k}\right) \mapsto h_{1} \cdot h_{2} \cdot \ldots \cdot h_{k}$ of the Cartesian product $H_{1} \times H_{2} \times \cdots \times H_{k}$ to $\Gamma$ is one-to-one. Then, by the $G^{\cdot m}$-inequality,

$$
\sum_{i}\left|\partial_{\mu_{i}}(Y)\right| \geq\left|\partial_{\mu}(Y)\right|
$$

where $\mu_{i}$ are the uniform probability measures on $H_{i}$ and $\mu$ is such measure on the product $H=H_{1} \cdot H_{2} \cdot \ldots \cdot H_{k} \subset \Gamma$. Since $|H|=\prod_{i}\left|H_{i}\right|$ under the "one-toone" assumption, one gets a good lower bound on $\sum_{i}\left|\partial_{\mu_{i}}(Y)\right|$ for biorderable (and sometimes more general) groups $\Gamma$.

(2) If $H \subset \mathbb{Z}$ admits no nontrivial relation

$$
\sum_{h \in H} n_{h} \cdot h=0,
$$


where $\left|n_{h}\right| \leq N$ for a given $N \geq 0$ and all $h \in H$, then every subset $Y \subset \mathbb{Z}$ of cardinality $|Y| \leq N$ satisfies

$$
|Y|^{k-1} \leq \prod_{h \in H}\left|\partial_{h}(Y)\right| .
$$

Proof. Let $C_{i}$ be the (Cayley) graph on the vertex set $Y$, where the edges are the pairs $\left(y, y+h_{i}\right) \in Y \times Y$ (for the group composition in $\mathbb{Z}$ depicted by “+”).

The number of the connected component in $C_{i}$, that is, the number $l_{i}$ of the maximal arithmetic $h_{i}$-progressions in $Y$, equals $\left|\partial_{h_{i}}(Y)\right|$. Furthermore, the family of the $k$ partitions $P_{i}$ of $Y$ into the components of $C_{i}$ is expanding in the sense of 5.7 due to the absence of the above relations. Hence, $|Y|^{k-1} \leq \prod_{i} l_{i}$ by the LoomisWhitney inequality. (In general, the inequality $|Y|^{k-1}>\prod_{i} l_{i}$ implies, for given positive integers $N_{i}$, either the existence, for some $i$, of an arithmetic $h_{i}$-progression in $Y$ of length $N_{i}$, or a nontrivial relation $\sum_{i} n_{i} h_{i}=0$ with $\left|n_{i}\right|<N_{i}$.)

Example. If $H=\left\{h_{i}\right\}$, where $\left|h_{i+1}\right|>N \sum_{j \leq i}\left|h_{j}\right|$, then the "no relation" condition is obviously satisfied.

The above easily generalizes to the sparse subsets $H$ in all $\Gamma$, where one may have a much larger $\left|\partial_{H}\right|$ with relatively small $H$ for non-Abelian groups $\Gamma$.

On the other hand, the exact evaluation of the max-profile is not apparent already for $\Gamma=\mathbb{Z}$ and $H=\{1,2, \ldots, k\}$ (where the problem pertains to the combinatorial number theory).

This suggests the

Dual isoperimetric problem. Find for every $r=1,2, \ldots$ and $0 \leq \alpha \leq 1$ a "minimal" subset $H \subset \Gamma$ such that

$$
\frac{|\partial|_{H}^{\max }(Y)}{|Y|} \geq \alpha
$$

for all $Y \subset \Gamma$ with $|Y| \leq r$, where "minimal" may refer to the cardinality of $Y$, to the radius of the minimal ball containing $H$, or to another notion of a size of a subset in $\Gamma$.

It seems that most (all?) proofs of lower bounds on the isoperimetric profiles of groups $\Gamma$ also deliver, under a close inspection, "small" $H$ giving "large" maxboundaries to all $Y \subset \Gamma$. 


\section{Linear algebraic entropic inequalities for normal group extensions}

We prove in this section coarse product inequalities for a suitably defined "filtered" (compare 1.11) entropic Følner functions of linear actions that apply, for example, to pure torsion groups where other techniques are not available.

7.1. Vertical families $\mathscr{L}$ of linear subspaces and $\mathscr{L}$-entropy. Let $L$ be a linear space over some field with a distinguished class $\mathscr{L}$ of what we call vertical linear subspaces in $L$, where we assume this class to be closed under finite and infinite intersections. If $D \subset L$ is a linear subspace, then we denote by $\mathscr{L} \mid D$ the induced class of subspaces in $D$ that are the intersection of $D$ with the subspaces from $\mathscr{L}$.

Tensor product example. Consider linear spaces $L_{\mathrm{hor}}$ and $L_{\mathrm{vert}}$ and take $L=$ $L_{\text {hor }} \otimes L_{\text {vert }}$, where $\mathscr{L}$ consists of the subspaces $L_{\text {hor }}^{\prime} \otimes L_{\text {vert }}$ for all linear subspaces $L_{\text {hor }}^{\prime} \subset L_{\text {hor }}$. Clearly, $\mathscr{L}$ consists of all subspaces invariant under the group of linear transformations of $L_{\text {vert }}$ naturally acting on $L$.

$\Gamma_{\text {vert }}$-examples. Let $L$ equals some $\Gamma$-invariant space of functions on a group $\Gamma$ and $\mathscr{L}$ consists of all $\Gamma_{\text {vert }}$-invariant subspaces in $L$ for some normal subgroup $\Gamma_{\text {vert }} \subset \Gamma$.

The quotient group $\Gamma_{\text {hor }}$ does not, in general, act on $L$ but it does act on $\mathscr{L}$ by projective transformations where each individual transformation comes from a linear transformation of $L$.

This class $\mathscr{L}$ is not always closed under complements: if $L_{1} \subset L_{2}$ for $L_{1}, L_{2}, \in \mathscr{L}$, then the quotient space $L_{2} / L_{1}$ is not always $\Gamma_{0}$-equivariantly isomorphic to any element of $\mathscr{L}$.

Here are two particular classes $\mathscr{L}$ where the complement always exists.

1. Support saturated class. A subspace $L_{1} \subset L$ is called support saturated if it contains all functions from $L$ which have supports contained in the support of $L_{1}$.

If $L$ consists of the functions with finite supports in $X$, then

the class of all $\Gamma_{\text {vert }}$-invariant support saturated subspaces admits complements corresponding to taking complements of $\Gamma_{\text {vert }}$-invariant subsets in $X$.

2. $l_{2}$-Spaces. If the underlying field is $\mathbb{R}$ or $\mathbb{C}$ and $L=l_{2}(X)$, then the class of all closed $\Gamma_{\text {vert }}$-invariant subspaces also admits (now just orthogonal) complements.

$\mathscr{L}$-length and $\mathscr{L}$-entropy. Take a finite dimensional linear subspace $D \subset L$ and consider all its $\mathscr{L}$-"partitions", i.e., decompositions into sums (spans) of non-zero independent subspaces $D=D_{1} \oplus D_{2} \oplus \cdots \oplus D_{i} \oplus \cdots \oplus D_{N}$ such that the associated filtrations are $\mathscr{L}$-vertical:

$$
D_{1} \oplus D_{2} \oplus \cdots \oplus D_{i} \in \mathscr{L} \mid D \quad \text { for all } i=1,2, \ldots N .
$$

Define the $\mathscr{L}$-length of $D$ as the maximal $N$ for which such "partition" exists, and the $\mathscr{L}$-entropy $\operatorname{ent} \mathscr{L}(D)$ as the supremum of the entropies of the finite measure spaces 
made of $N$ atoms of weights $d_{i}=\left|D_{i}\right|=\operatorname{rank}\left(D_{i}\right)$ over all $\mathscr{L}$-"partitions" of $D$ for all $N=1,2, \ldots$

Clearly,

$$
|D| \geq \operatorname{length}_{\mathscr{L}}(D) \geq \exp (\operatorname{ent} \mathscr{L}(D))
$$

for all $\mathscr{L}$ and $D$.

Example. If $\mathscr{L}$ consists of all linear subspaces in $L$ then $\mathscr{L}$-length of $D$ equals $|D|$ and ent $\mathscr{L}(D)=\log |D|$, but if $\mathscr{L}=\left\{L_{\text {vert }}^{\prime} \otimes L_{\text {hor }}\right\}$ for an infinite dimensional space $L_{\text {vert }} \supset L_{\text {vert }}^{\prime}$, then generic finite dimensional subspaces $D \subset L=L_{1} \otimes L_{2}$ have $\operatorname{ent}_{\mathscr{L}}(D)=0$.

$\mathscr{L}$-boundary. Given a finite dimensional linear subspace, define the $g \mid \mathscr{L}$-boundary of $D$ for a linear transformation $g$ of $L$ preserving $\mathscr{L}$ by

$$
\left|\partial_{g \mid \mathscr{L}}\right|(D)=\sup _{L^{\prime} \in \mathscr{L}}\left|D \cap L^{\prime} / g^{-1}(D)\right| .
$$

Clearly, this equals the ordinary boundary $\left|\partial_{g}(D)\right|$ if $D \in \mathscr{L}$; in general,

$$
\left|\partial_{g \mid \mathscr{L}}\right|(D) \leq\left|\partial_{g}(D)\right| .
$$

Logarithmic filtration lemma. Let $G$ be a set of transformations of $L$ such that $\mathscr{L}$ is $G$-invariant, yet it contains no non-zero $G$-invariant subspace. If a finite dimensional subspace $D$ is contained in some $L_{D}^{\prime} \in \mathscr{L}$ and

$$
\left|\partial_{g \mid \mathscr{L}}\right|(D) \leq \varepsilon|D|
$$

for all $g \in G$, then there is a descending sequence of subspaces $D=D_{0} \supset D_{1} \supset$ $D_{2} \supset \cdots \supset D_{i} \supset \cdots \supset 0$, where each $D_{i}=D \cap L_{i}^{\prime}$ for $L_{i}^{\prime} \in \mathscr{L}$, such that

$$
\left|D / D_{i}\right| \leq 2\left|D / D_{i-1}\right|+\varepsilon
$$

for all $i=1,2, \ldots$

Proof. Take a sequence $\gamma_{i}$ of invertible linear transformations of $L$, where $\gamma_{0}=\mathrm{id}$, $\gamma_{i} \cdot \gamma^{-1} \in G$ for all $i$, and every transformation in the (linear) transformation group generated by $G$ appears as some $\gamma_{i}$ in the sequence. Then the sequence

$$
D_{i}^{\prime}=D \cap \gamma_{0}\left(L_{D}^{\prime}\right) \cap \gamma_{1}\left(L_{D}^{\prime}\right), \ldots, \gamma_{i}\left(L^{\prime}\right) .
$$

Remark. If $\mathscr{L}$ is associated with a normal subgroup $\Gamma_{\text {vert }}$ in $\Gamma$ generated by $G$, then the inequality $(2+\varepsilon)$ looks abysmally weak compared to the corresponding (equally obvious) combinatorial intersection inequality (compare 1.4)

$$
\left|Y \backslash Y_{i}\right| \leq \varepsilon,
$$

where $Y_{i}=Y \cap Y_{i}^{\prime}$ for $\Gamma_{\text {vert }}$-invariant subsets in $\Gamma$. It is unclear to me if/when 2 can be removed from $(2+\varepsilon)$ for linear actions. 
$\frac{1}{4}$-entropy corollary. If

$$
|\partial|_{G \mid \mathscr{L}}^{\max }(D):=\max _{g \in G}(D) \leq \varepsilon|D|
$$

for $\varepsilon \leq \frac{1}{4}$, then

$$
\operatorname{ent}_{\mathscr{L}}(D) \geq \frac{1}{4} . \quad\left(\text { ent } \geq \frac{1}{4}\right)
$$

Remark. The corresponding (also obvious) bound on the length of $D$,

$$
\operatorname{length}_{\mathscr{L}}(D) \geq \log _{3}(\varepsilon)-2,
$$

looks somewhat better, but (ent $\geq \frac{1}{4}$ ), albeit independent of $\varepsilon$, better behaves under induction, as we shall see below.

7.2. $\mathscr{L}$-entropic Følner functions. Given a countable group with a (usually generating) subset $G \subset \Gamma$, consider all countable overgroups $\widetilde{\Gamma} \rightarrow \Gamma \rightarrow 1$ with the kernel denoted $\Gamma_{\text {vert }} \subset \widetilde{\Gamma}$ and let $\tilde{L}$ stand either for the space of $\mathbb{F}$-valued functions on $\widetilde{\Gamma}$ with finite supports on $\widetilde{\Gamma}$ for a given field $\mathbb{F}$, or for the space of the complex $l_{2}$-functions on $\widetilde{\Gamma}$.

Denote by $\widetilde{\mathscr{L}}$ the family of $\Gamma_{\text {vert }}$-invariant spaces $\tilde{L}^{\prime}$ of functions on $\widetilde{\Gamma}$ with either finite projections of their supports to $\Gamma$, or of $l_{2}$-spaces with finite von Neumann $\Gamma_{\text {vert-dimensions. }}$ -

Define the internal $G \mid \mathscr{L}$-boundary of a $\widetilde{D} \subset \tilde{L}$ by

$$
\left|\partial_{\widetilde{G} \mid \mathscr{L}}\right|(D)=\sup _{L^{\prime} \in \mathscr{L}}\left(\left|D \cap L^{\prime} \cap \bigcap_{g \in \widetilde{G}} \gamma^{-1}(D)\right|\right),
$$

where $\widetilde{G}$ denotes some lift of $G$ to $\widetilde{\Gamma}$. Note that the definition is independent of a lift and $\left|\partial_{\widetilde{G} \mid \mathscr{L}}\right|(D) \geq\left|\partial_{\widetilde{G} \mid \mathscr{L}}^{\max }\right|(D)$.

Define the entropic Følner function $\widetilde{F}_{\bullet}(n)=\widetilde{F}_{\bullet}(n ; \Gamma, G)$ as the maximal •-convex function such that

$$
\exp (\operatorname{ent} \mathscr{L}(\widetilde{D})) \geq \widetilde{F}_{\text {. }}(n)
$$

for all finite dimensional subspaces $\widetilde{D} \subset \tilde{L}$ satisfying

$$
\left|\partial_{\widetilde{G} \mid \tilde{\mathscr{L}}}\right|(D) \leq \frac{1}{n}|D|
$$

for all overgroups $\widetilde{\Gamma}$ and all finite dimensional subspaces $\widetilde{D} \in \tilde{L}$.

If there are several $G_{i}$, let

$$
\widetilde{F}_{\bullet}^{\max _{i}}(n)=\widetilde{F}_{\bullet}\left(n_{i} ; \Gamma, G_{i}\right)
$$


be the maximal $\bullet$-convex function such that

$$
\exp (\operatorname{ent} \mathscr{L}(\widetilde{D})) \geq \tilde{F}_{\bullet}\left(n_{i}\right)
$$

for all finite dimensional subspaces $\tilde{D} \subset \tilde{L}$ satisfying

$$
\left|\partial_{\widetilde{G_{i}}}\right| \widetilde{\mathscr{L}}\left|(D) \leq \frac{1}{n_{i}}\right| D \mid
$$

for all overgroups $\widetilde{\Gamma}$ and all finite dimensional subspaces $\widetilde{D} \in \tilde{L}$, where the --convexity is understood as the convexity of $\log \left(F\left(1 / \varepsilon_{i}\right)\right)$ as a function of the vectorvariable $\left\{\varepsilon_{i}\right\}$.

Super-multiplicativity lemma. Let

$$
1 \rightarrow \Gamma_{1} \rightarrow \Gamma \rightarrow \Gamma_{2} \rightarrow 1
$$

and let $G_{1}=G \cap \Gamma_{1}$ and $G_{2} \subset \Gamma_{2}$ be the projection of a given $G \in \Gamma$ to $\Gamma_{2}$.

Then

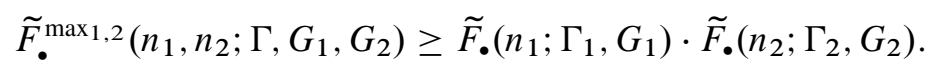

Proof. The $\tilde{\mathscr{L}}_{\text {- }}$ partition" of a given $D \subset \tilde{L}$ is constructed in two steps.

First we let $\widetilde{\mathscr{L}}_{1} \subset \widetilde{\mathscr{L}}$ be the subclass of subspaces that are invariant under $\Gamma_{1}$ or rather under the pullback of $\Gamma_{1}$ to $\widetilde{\Gamma}$, and take the $\widetilde{\mathscr{L}}_{1}$-"partition" $D=\bigoplus_{i} D_{i}$ of the maximal entropy that is bounded from below with the second Følner function by $\log \left(\tilde{F}_{.}\left(n ; \Gamma_{2}, G_{2}\right)\right)$.

Then we partition each $D_{i}$ with $\mathscr{L}$. Since the entropy is additive (see Section 5) and the boundary $\partial_{G \mid \mathscr{L}}$ is "slice-wise"-additive, the resulting entropy is bounded from below by the sum of the log's of the first and the second Følner functions by the -convexity of the first one, as in 5.11 .

Remark. This argument, unlike the one in 5.11, does not use the Shannon inequality.

By combining this Lemma with the above $\frac{1}{4}$-Corollary, we arrive at the following.

Chain extension inequality. Let $1=\Gamma_{0} \subset \Gamma_{1} \subset \cdots \subset \Gamma_{i} \subset \cdots \subset \Gamma_{N} \subset \Gamma$ be a normal sequence, i.e., each $\Gamma_{i}$ is normal in $\Gamma_{i+1}$, and let $G_{i} \subset \Gamma_{i}$ be subsets such that the image of $G_{i}$ in $\Gamma_{i} / \Gamma_{i-1}$ generates an infinite subgroup for all $i=1,2, \ldots$ Then

$$
\widetilde{F}_{.}^{\max _{i}}\left(n_{i} ; \Gamma, G_{i}\right) \geq \exp \left(\frac{N}{4}\right)
$$

for $n_{i} \geq 4$. 
Since the $\widetilde{F}$ minorizes the ordinary linear algebraic Følner function $F_{*}$ (for functions with bounded supports and $l_{2}$-functions respectively), we conclude, as in 6.1 , by applying the above to Cartesian products of infinite groups, that

the $l_{2}$-Folner function $F_{l_{2}}$ as well as $F_{*}$ for finitely supported functions with values in an arbitrary field of the wreath product $\Gamma$ of $\Gamma_{1}$ and $\Gamma_{2}$ are bounded from below in terms of the growth function of $\Gamma_{2}$ by

$$
F_{l_{2}}(n), F_{*}(n) \succ \exp \left(\frac{1}{4} G_{\circ}\left(n ; \Gamma_{2}\right)\right),
$$

provided the group $\Gamma_{1}$ is infinite (finitely generated).

There are amenable pure torsion groups, e.g., the wreath products of Grigorchuk groups, with growth $G_{\circ}(n) \sim 2^{n}$ and where $F_{*}(n)$ and $F_{l_{2}}(n)$ are $\succ 2^{2^{n}}$.

Questions. Do the higher iterates of wreath products of the Grigorchuk groups have faster growing $F_{*}$ and $F_{l_{2}}$ ? Are there amenable groups (torsion or no torsion) with $F_{l_{2}}(n)$ growing faster than $2^{2^{n}}$ ? What are the Følner functions of the iterated wreath products acting on the spaces of decaying functions with values in ultrametric fields? (If $\Gamma_{1}$ contains an element of infinite order, then the Følner function of $\Gamma=\Gamma_{1}^{2 \Gamma_{2}}$ for decaying functions grows $\succ \varepsilon(1+\varepsilon)^{G_{\circ}\left(n ; \Gamma_{2}\right)}, \varepsilon>0.1$ by 6.1$)$.

Application to Grigorchuk groups. If a finitely generated group $\Gamma$ contains a subgroup commensurable to $\Gamma \times \Gamma$ (e.g. a suitable Grigorchuk group), then, by the super-multiplicativity,

$$
\widetilde{F}(n ; \Gamma) \geq \varepsilon_{\Gamma} \cdot\left(\widetilde{F}\left(\varepsilon_{\Gamma} n ; \Gamma\right)\right)^{2}
$$

for some strictly positive $\varepsilon_{\Gamma}$. It follows that

the Folner functions $F_{*}$ and $F_{l_{2}}$ of such $\Gamma$ grow at least as fast as $\exp \left(n^{\alpha}\right)$ for some $\alpha=\alpha_{\Gamma}>0$.

Isoperimetry in vertical Grassmannians. Let us regard a class $\mathscr{L}$ as a subspace in the Grassmannian $\operatorname{Gr}(L)$ of linear subspaces $L^{\prime}$ of a linear space $L$. The notion of isoperimetry makes sense for a projective action of a group $\Gamma$ on $\mathscr{L}$, whenever there is some notion of "dimension" or "rank" for linear subspaces $L$ ' $\in \mathscr{L}$.

Such a notion is provided in the $l_{2}$-environment by the von Neumann $\Gamma_{\text {vert }}{ }^{-}$ dimension, when all $L^{\prime} \in \mathscr{L}$ are acted upon by a group $\Gamma_{\text {vert }}$ as in the above examples. Furthermore, if $\Gamma_{\text {vert }}$ is amenable, there is the asymptotic rank defined in 1.10.

It seems that such projective actions on sub-Grassmannians constitute a correct general setting for the "linearized isoperimetry". For example, the above entropic argument applies here and delivers the corresponding isoperimetric inequalities. But I have not looked into the subject matter beyond this point. 


\section{Examples of group algebras with fast and with slow growing Følner functions}

We start with a bound on the isoperimetric profiles and Følner functions of certain group algebras and then construct groups where the linear algebraic profiles grow much slower than the combinatorial ones. Also we construct groups with arbitrarily fast growing Følner functions of their group algebras.

8.1. An upper bound on $I_{*}$ for split extensions. Consider an exact sequence of groups

$$
1 \rightarrow \Theta \rightarrow \Gamma \rightarrow \Gamma_{0} \rightarrow 1,
$$

where $\Gamma$ (and, hence, $\Gamma_{0}$ ) is finitely generated.

If the sequence splits by some embedding $\Gamma_{0} \rightarrow \Gamma$ and the group $\Theta$ is locally finite (i.e., every finitely generated subgroup in $\Theta$ is finite), then the isoperimetric profile $I_{*}(r ; A)$ of the group algebra $A$ of $\Gamma$ is equivalent to the profile $I_{*}\left(r ; A_{0}\right)$ of the group algebra $A_{0}$ of $\Gamma_{0}$ with an arbitrary coefficient field $\mathbb{F}$. In particular, if $\Gamma_{0}=\mathbb{Z}$, then $I_{*}(r ; A)$ is bounded.

Proof. Choose a symmetric finite generating set $G=G_{0} \cup G_{1} \subset \Gamma$, where $G_{0} \subset$ $\Gamma_{0} \subset \Gamma$ and $G_{1} \subset \Theta$. Take a linear space $D_{0} \subset A_{0}$ of $\mathbb{F}$-valued functions on $\Gamma_{0}$ with supports in a finite subset $Y_{0} \subset \Gamma_{0}$. Denote by $\Theta_{1} \subset \Theta$ the (finite!) subgroup generated by $y g_{1} y^{-1}$ for all $y \in Y_{0}$ and $g_{1} \in G_{1}$.

Let $a_{1} \in A$ be an $\mathbb{F}$-valued function on $\Gamma$ which equals 1 on $\Theta_{1} \subset \Theta \subset \Gamma$ and vanishes everywhere else on $\Gamma$. Observe that $a_{1}$ is invariant under all above $y g_{1} y^{-1}$.

The embedding $\Gamma_{0} \rightarrow \Gamma$ makes $\Gamma_{0}$ and hence its group algebra $A_{0}$ act on functions on $\Gamma$; we denote by $D$ the linear span of the functions $d * a_{1}$ on $\Gamma$ for all $d \in D_{0}$ where " $*$ " stands for the (convolution) product in the group algebra $A$ of $\Gamma$.

Each $g_{1} \in G_{1} \subset \Theta$ trivially acts on $y * a_{1}$ for all $y, y^{-1} \in Y_{0}$. Indeed, $g_{1}=$ $y \theta_{1} y^{-1}$ for $\theta_{1}=y^{-1} g_{1} y \in \Theta_{1}$ and $y \in Y_{0}$. Then

$$
g_{1} *\left(y * a_{1}\right)=\left(y \theta_{1} y^{-1}\right) *\left(y * a_{1}\right)=\left(y \theta_{1}\right) * a_{1}=y * a_{1},
$$

where $\theta_{1}$, since it is contained in $\Theta_{1}$, acts trivially on $a_{1}$. Therefore, the space $D$ is invariant, actually fixed, under the action of all $g_{1} \subset G_{1}$, because it is contained in the span of the $y * a_{1}$ for $y \in Y_{0}$.

It follows that

$G$-boundary of $D$ equals its $G_{0}$-boundary.

Since $\Gamma_{0}$ freely acts on $\Gamma$ and every $\Gamma_{0}$-orbit meets $\Theta \subset \Gamma$ at a single point, the action of $\Gamma$ on the span of the orbit of every non-zero function $a$ on $\Gamma$ with support in $\Theta$ is isomorphic to the action of $\Gamma_{0}$ on the orbit of the unit in its group algebra, 
which is the span of the orbit of the unit. This isomorphism sends $D \rightarrow D_{0}$ and $\partial_{G_{0}}(D) \rightarrow \partial_{G_{0}}\left(D_{0}\right)$; hence,

$$
|D|=\left|D_{0}\right| \quad \text { and } \quad\left|\partial_{G}(D)\right|=\left|\partial_{G_{0}}(D)\right|=\left|\partial_{G_{0}}\left(D_{0}\right)\right| .
$$

This shows that $I_{*}$ of $\Gamma$ is bounded by $I_{*}$ of $\Gamma_{0}$, because $D_{0}$ is an arbitrarily chosen subspace in $A_{0}$.

The proof is concluded by observing that the reverse inequality follows from $(+)_{*}$ in 1.5 .

Examples. (1) Take the wreath product $\Gamma=\Gamma_{1}^{2 \Gamma_{2}}$, where $2 \geq\left|\Gamma_{1}\right|<\infty$. Then the combinatorial $F \varnothing l$ lner function of $\Gamma$ satisfies

$$
F_{\circ}(n ; \Gamma) \succ(1+\alpha)^{F_{\circ}\left(n ; \Gamma_{2}\right)}
$$

for $\alpha>0$, while the Følner function of the group algebra of $\Gamma$ is bounded by

$$
F_{*}(n ; \Gamma) \prec F_{\circ}\left(n ; \Gamma_{2}\right) .
$$

Remark. The latter agrees with the bound

$$
F_{*}\left(n ; \Gamma_{1}^{2 \Gamma_{2}}\right) \prec F_{*}\left(n ; \Gamma_{2}\right) F_{*}\left(n ; \Gamma_{1}\right)^{F_{\circ}\left(n ; \Gamma_{2}\right)},
$$

since $F_{*}\left(n ; \Gamma_{1}\right) \equiv 1$ for finite groups $\Gamma_{1}$.

(2) There are groups $\Gamma$ with two generators that are (necessarily split) extensions of locally finite groups $\Theta$,

$$
1 \rightarrow \Theta \rightarrow \Gamma \rightarrow \mathbb{Z} \rightarrow 1
$$

where one can have an arbitrary fast growing Følner function of $\Gamma$ (see 8.2 below). Yet, by the above criterion, their groups algebras have bounded isoperimetric functions.

On extensions of finitely generated groups. If both groups $\Gamma_{0}$ and $\Theta$ in a (not necessarily split) exact sequence $1 \rightarrow \Theta \rightarrow \Gamma \rightarrow \Gamma_{0} \rightarrow 1$ are finitely generated and infinite, then the max-isoperimetric profile of the group algebra of $\Gamma$ is unbounded; moreover

$$
I_{*}^{\max }(r: \Gamma, G) \succ \log (r)
$$

by the $\mathscr{L}$-length inequality in 7.1 .

Questions. Let an infinite finitely generated group $\Gamma$ have $I_{*}$ bounded. Is it then a $\mathbb{Z}$-extension of a locally finite group?

Does any of the following conditions help?

(1) $\Gamma$ is a torsion group; 
(2) $\Gamma$ contains an infinite finitely generated subgroup of infinite index;

(3) $\Gamma$ contains an element of infinite order;

(4) $\Gamma$ has no torsion.

If $\Gamma$ has no torsion, does then the polynomial bound on the Følner function, $F_{*}(r) \preceq r^{k}$ for some $k>1$, imply that $\Gamma$ is virtually nilpotent?

8.2. Amenable groups with fast growing Følner functions. Let $F_{X}$ denote the free group on a set $X$ of generators and let $[Y]_{k} \subset F_{X}$ be the set of the commutators $\left[\ldots\left[\left[x_{1}, x_{2}\right], x_{3}\right] \ldots x_{k}\right]$ for all $k$-long sequences $x_{i} \in Y$.

Denote by $F_{X}(p)$ the quotient group of $F_{X}$ by the relation $x^{p}=1$ for some prime number $p$ and all $x \in X$ (that is the free product of $X$ copies of $\mathbb{Z}_{p}$ ) and observe that

1. If an element in $F_{X}(p)$ is representable by a word $y_{1}^{i_{1}} \cdot y_{2}^{i_{2}} \cdot \ldots \cdot y_{k}^{i_{k}}$, where the neighboring $y_{i} \subset Y$ are not equal and where no $i_{j}$ is divisible by $p$, then the image of $w$ under the composed (surjective) homomorphism $h: F_{X} \rightarrow F_{Y}(p) /[Y]_{k}$,

$$
F_{X} \rightarrow F_{Y} \rightarrow F_{Y}(p) \rightarrow F_{Y}(p) /[Y]_{k}
$$

is $\neq 1$.

Here is the (standard)

Proof. Assume $X=Y$ and let $W_{k}$ be the $\mathbb{F}_{p}$-linear space (formally) spanned by the irreducible words $w=y_{1}^{j_{1}} \cdot y_{2}^{j_{2}} \cdot \ldots \cdot y_{l}^{j_{l}} \subset F_{X}$, where all $j \geq 0$ and the total degree of $w$ satisfies $\operatorname{deg}(w)=j_{1}+j_{2}+\cdots+j_{l} \leq k$.

Let $A_{y}$ be the (nilpotent) linear operators on $L_{k}$ defined by their values at $w \in L_{k}$ as follows: $A(w)=y w$ for $\operatorname{deg}(w)<k$, and $A(w)=0$ otherwise.

The map $y \mapsto 1-A_{y}$ extends to a homomorphisms of $F_{Y}(p) /[Y]_{k}$ to the group of linear transformations of $W_{k}$, where each $y^{-1}$ goes to the finite sum $1+A_{y}+$ $A_{y}^{2}+\cdots+A_{y}^{k}$, since $A_{y}^{k+1}=0$. Then the non-equality $h(w) \neq 1$ follows from non-vanishing of the product operator $A_{y_{1}}^{j_{1}} \cdot A_{y_{2}}^{j_{2}} \cdot \ldots \cdot A y_{l}^{j_{l}}$.

This $k$-acyclicity/divergence of $F_{Y}(p) /[Y]_{k}$ implies the following lower bound on the Følner function of the (finite for $p<\infty$ ) group $F_{Y}(p) /[Y]_{k}$ for the (joint, see 1.1) boundary $\partial_{Y}$, where we regard $Y$ as a (generating) subset in $F_{Y}(p) /[Y]_{k}$ :

$$
F_{\circ}(n) \geq((|Y|-1)(q-1))^{\alpha k}
$$

for $q=\min (n, p)$ and some $\alpha \geq 1 / 4$.

Remark. One can do better including estimates on the $E_{\circ}$ and $P_{\circ}$ functions of the group $N(k)=F_{Y}(p) /[Y]_{k}$ with respect to the cyclic subgroups generated by $y \in Y$, while we shall need below only the (rough but uniform) bound

$$
F_{\circ}(n)=F_{\circ}(n ; N(k), Y) \geq k \text { for all } p \geq 3 \text { and }|Y| \geq 2
$$

for all $n \geq 1$, which is stated below in a form that is convenient for our examples. 
2. Let $k(Y)$ be a function (rather than a number) on the subsets $Y \subset X$ with values $1,2,3, \ldots, \infty$ such that $Y_{1} \subset Y_{2} \Longrightarrow k\left(Y_{1}\right) \leq k\left(Y_{2}\right)$. Let $k_{2}(Y)(\leq k(Y))$ be the maximum of $k\left(Y^{\prime}\right)$ over all subsets $Y^{\prime} \subset Y$ of cardinality $\left|Y^{\prime}\right|=2$. Let $N(k)$ be the factor group of $F_{X}(p)$ obtained with the above (commutator) relations $\left[Y_{k(Y)}\right]$ for all $Y \subset Y$, where $\left[Y_{k(Y)}\right]$ for $k(Y)=\infty$ means "no relation". Then

$$
F_{\circ}(n)=F_{\circ}(n ; N(k), Y) \geq k_{2}(Y)
$$

for all $Y \subset X$ with $|Y| \geq 2$, all $p \geq 3$ and $n \geq 1$.

Next let $X$ be acted by a group $\Gamma$ with a given finite generating set $G \subset \Gamma$ and let the function $k(Y)$ be $\Gamma$-invariant.

Then $\Gamma$ acts by automorphisms on $N(k)$ and thus, diagonally, on $N(k) \times X$. The group $N(k)$ also acts on the product $N(k) \times X$ by translations on the first factor, and the group generated by the two actions is denoted by $N(k) \ltimes \Gamma$. If the action of $\Gamma$ on $X$ is transitive, then the group $N(k) \ltimes \Gamma$ is finitely generated, namely, by the subset $\left\{x_{0}\right\} \cup G \subset N \ltimes \Gamma$ for an arbitrary $x_{0} \in X \subset N \ltimes \Gamma$,

3. Let $\operatorname{Diam}_{G}(Y)$ be the minimal number $m$ such that every point $y_{1} \in Y$ can be moved to any other point $y_{2} \in Y$ by some $\gamma \in G^{\cdot m}$, i.e., $\gamma\left(y_{1}\right)=y_{2}$, where we assume that $G$ is symmetric (under $\gamma \leftrightarrow \gamma^{-1}$ ) and contains id. Denote by $k_{2}(m)$ the supremum of $k_{2}(Y)$ over all $Y \subset X$ with $\operatorname{Diam}_{G}(Y)=m$.

The Folner function of the action of $N(k) \ltimes \Gamma$ on $N(k) \times X$ with the generating subset $\left\{x_{0}\right\} \cup G \subset N(k) \ltimes \Gamma$ satisfies

$$
F_{\circ}(m n ; N(k) \times X) \geq k_{2}(m)
$$

for $n \geq 1$ and all $m=2,3, \ldots$. In particular, if $k(Y)$ depends only on $m=$ $\operatorname{Diam}_{G}(Y)$, then $k_{2}=k$ and

$$
F_{\circ}(m n ; N(k) \times X) \geq k(m) .
$$

Proof. Combine the above with $(n / m)$ in 1.9 .

This shows that one can make $F(n)$ grow arbitrarily fast with a fast growing function $k(m)$ and, at the same time, have $N(k)$ locally nilpotent; thus $N(k) \ltimes \Gamma$ is amenable if $\Gamma$ is amenable.

Here is the summary.

Example: amenable groups with $\boldsymbol{F}_{\circ} \geq \boldsymbol{\kappa}(\boldsymbol{n})$. Let $X=\Gamma$ be an amenable group with a finite generating subset $G \subset \Gamma$ and let $\kappa(n), n=1,2, \ldots$, be an arbitrary monotone increasing $\mathbb{Z}_{+}$-valued function. Then the group $N(k) \ltimes \Gamma$ for $k(Y)=$ $\kappa\left(\operatorname{Diam}(Y)^{2}\right), Y \subset \Gamma$, is finitely generated amenable and

$$
F_{\circ}(n ; N(k) \ltimes \Gamma, G) \geq \kappa(n)
$$

for all $n \geq 1$. 
Examples with the linear algebraic Følner functions $\boldsymbol{F}_{*}$. If $p=\infty$, then the group $N(k)$ is orderable and the above yields the lower bound

$$
F_{*}(n ; N(k) \ltimes \Gamma) \geq \kappa(n)
$$

for the (action of $N(k) \ltimes \Gamma$ on) functions with compact supports on $N(k) \ltimes \Gamma$ with values in an arbitrary field (where one does not need $\Gamma$ to be orderable).

On the other hand, if $p \leq \infty$, then

$$
F_{*}(n ; N(k) \ltimes \Gamma) \leq F_{\circ}(n ; \Gamma),
$$

while $F_{\circ}(n ; N(k) \ltimes \Gamma)$ can grow as fast as one wishes.

Remark and questions. (a) The existence of groups $N \ltimes \mathbb{Z}$ with arbitrarily fast growing $F_{\circ}$, where $N$ is a locally finite group, is indicated in [9], and groups of intermediate growth with arbitrarily fast growing $F_{\circ}$ are constructed in [10].

(b) It is not hard to give an upper bound on $F_{\circ}$ for the above group and to observe that

$$
F_{*}(n ; N(k) \ltimes \Gamma) \sim \kappa(n)
$$

for the functions $\kappa(n)$ with sufficiently fast growing derivatives.

(c) Can one achieve the above with a construction (invariant under all permutation of $X$ ) with $k(Y)$ depending on the cardinality rather than the diameter of $Y$ ? (Possibly, this can be done with the existence theorem for non-Abelian free subgroups in groups with universal commutator relations, [3]).

(d) Is there an universal bound on the asymptotic growth of the Følner functions of finitely presented amenable groups by a recursive (primitively recursive?) function? (Maybe there is such a bound in every given recursive class of presentations?) Or, at another extreme, are there finitely presented amenable groups with so fast growing $F_{\circ}(n)$ such that their amenability is unprovable in arithmetic? (An enticing possibility would be this situation for the Thompson group.)

\section{9. $E_{\mathrm{o}}$-functions and isoperimetric inequalities for wreath powers of partitions and groups}

The isoperimetric inequalities in the previous section are similar to those for the wreath product of groups that were obtained earlier in an asymptotically sharp form in [9] with an (implicate) use of $E_{\circ}$-functions. We shall present below a translation of the argument from [9] to the language of partitions that provides a quantitative (better constants) improvement of the isoperimetric inequalities for iterated wreath products of groups and a qualitative (asymptotic behavior) improvement for infinitely iterated wreath products. 
9.1. Wreath powers of sets. Let $Z$ be a discrete set with a distinguished family 8 of subsets (slices) $S \subset Z$, and define the 2 -space associated to $\delta$, denoted $Z_{l}$, which is the set of the pairs $(S, z)$ for $S \in \delta$ and $z \in S$.

The simplest (and the largest) such $Z_{2}$ is obtained with $\delta=2^{Z}$, the family of all subsets in $Z$.

The set $Z_{2}$ naturally carries two partitions $P_{1}$ and $P_{2}$ : the slices of $P_{1}$ are obtained by varying $z$ in the pair $(S, z)$ within $S$, while the slices of $P_{2}$ consist of the pairs $(S, z)$ with fixed $z$ and all $S \ni z$.

Basis example. Let $Z=X^{I}$ with $\delta$ being the family of the "coordinate lines". In this case we write $Z_{2}=Z \times I=X^{2 I}$ and denote the two corresponding partitions of $X^{2 I}$ by $P_{1}=P_{X}$, where all slices are copies of $X$, and by $P_{2}=P_{I}$, where the slices are copies of $I$.

Recall that this 8 is Shannon: the geometric mean of the cardinalities of the 8 -slices of every $Y \subset Z$ is bounded by the cardinality of $Y$,

$$
\prod_{S}|S \cap Y|^{\frac{1}{|Y|}} \leq|Y|
$$

where the product is taken over the slices $S \in \mathcal{\&}$ with non-empty intersections $S \cap Y$.

Evaluation of $\boldsymbol{E}_{\circ}, \boldsymbol{P}_{\circ}$ and $\boldsymbol{F}_{\odot}$ for $\boldsymbol{Z}_{\imath}$. If 8 is Shannon, then

$$
\begin{gathered}
E_{\circ}\left(L_{1}, L_{2} ; P_{1}, P_{2}\right)=L_{2} \cdot L_{1}^{L_{2}}, \\
P_{\circ}\left(n_{1}, n_{2} ; Z_{\curlywedge} / P_{1}, Z_{\curlywedge} / P_{2}\right)=n_{2} \cdot n_{1}^{n_{2}}
\end{gathered}
$$

and

$$
F_{\odot}\left(F_{1}\left(n_{1}\right), F_{2}\left(n_{2}\right)\right) \geq d_{2} F_{2}\left(\varepsilon_{2} n_{2}\right)\left(d_{1} F_{1}\left(\varepsilon_{1} n_{1}\right)\right)^{d_{2} F_{2}\left(\varepsilon_{2} n_{2}\right)}, \quad\left(F^{2 F}\right)
$$

where

$$
d_{i}=\frac{1-\varepsilon_{1}-\varepsilon_{2}}{1-\varepsilon_{i}}
$$

for arbitrary positive numbers $\varepsilon_{i}, i=1,2$, satisfying

$$
\varepsilon_{1}+\varepsilon_{2}<1 .
$$

Furthermore, if $F_{2}(n)=$ const $\cdot n$ and $F_{1}(n)$ is $\bullet$-convex, then

$$
F_{\odot}\left(F_{1}\left(n_{1}\right), F_{2}\left(n_{2}\right)\right) \geq F_{2}\left(n_{2}\right) F_{1}\left(n_{1}\right)^{F_{2}\left(n_{2}\right)} .
$$

Proof. If a subset $Y \subset Z_{2}$ has the $P_{1}$-slices of cardinalities $\geq L_{1}$ and the $P_{2}$-slices of cardinalities $\geq L_{2}$ then its projection $Y^{\prime}$ to $Z$ has at least $L_{2}$ slices of cardinalities $\geq L_{1}$ at each point in $Z$. Then, by the definition of "Shannon", $\left|Y^{\prime}\right| \geq L_{1}^{L_{2}}$ and 
$|Y| \geq L_{2} \cdot L_{1}^{L_{2}}$. This proves $\left(E_{\circ}\right)$ and, similarly, $\left(P_{\circ}\right)$ follows from the $A^{B}$-inequality (see 5.3, 5.7).

To prove $\left(F^{2 F}\right)$ we take a $Y \subset Z_{2}$ with

$$
\sum_{S \in P_{1}} I_{1}(|S \cap Y|) \leq \frac{1}{n_{1}}|Y|
$$

and

$$
\sum_{S \in P_{2}} I_{2}(|S \cap Y|) \leq \frac{1}{n_{2}}|Y|
$$

for the "isoperimetric profiles" $I_{i}$ associated with $F_{i}$ and let $Y_{i} \subset Y, i=1,2$, be the union of the $P_{i}$-slices of cardinalities $\geq F_{i}\left(\left(1-\varepsilon_{i}\right) n_{i}\right)$. Then the intersection $Y_{\bullet}=Y_{1} \cap Y_{2}$ is non-empty and has "large average measures" of $P_{1}$ - and $P_{2}$-slices according to $\left(\left[\bigcap_{i} / P_{i}\right)\right]$ in 4.5 , p. 538. Then $\left(A^{B}\right)$ applies with $\left.A=d_{1} F_{1}\left(\varepsilon_{1} n_{1}\right)\right)$ and $B=d_{2} F_{2}\left(\varepsilon_{2} n_{2}\right)$.

Finally we turn to $F_{\text {. }}$ and observe that the inequality

$$
\sum_{S \in P_{2}} I_{2}(|S \cap Y|) \leq \frac{1}{n_{2}}|Y|
$$

implies that the number of the $P_{2}$-slices of $Y$ is bounded by

$$
\left|P_{2} \cap Y\right| \leq \frac{1}{n_{2}}|Y| .
$$

The claim follows by the $\bullet$-convexity of $F_{\circ}\left(n ; \Gamma_{1}\right)$.

Remarks. (a) The evaluation of the $E_{\circ}$ function for $X^{2 I}$ (and for $Z$ ? associated to an expanding family of partitions $P_{i}$ in general) can be seen directly by counting $P_{i}$-chains in $Z$ (see [9] where it is done for wreath products of groups and graphs in the language of hypergraphs rather than partitions).

(b) There are further constructions and partitions associated to power spaces $X^{I}$, e.g., the 2-spaces associated to the "plane" rather than "line" partitions. Then one can take Cartesian powers of $Z$ ? that are products of the original $P_{i}$. Evaluation of the invariants the resulting families of partitions remains an open problem.

(c) It is unclear, in general, if there is a Shannon type inequality for measures $\mu$ on (rather than subsets in) $Z_{l}$, except for the case of the atoms in each $P_{2}$-slice $S$ having equal weights, denoted $\mu_{S}$, where the Shannon inequality relates the entropy of $P_{1}$ and the measure of $P_{2}$ to the weights $S \mapsto \mu_{S}$,

$$
\operatorname{ent}_{\mu}(X) \geq \operatorname{ent}_{\mu}\left(Z_{\imath}\right)=\frac{\mu\left(Z_{\imath}\right)}{\sum_{S \in P_{2}} \mu_{S}}+\log \frac{\mu\left(Z_{\imath}\right)}{\sum_{S \in P_{2}} \mu_{S}} .
$$

But it is doubtful that the concepts of "measure" and/or "entropy" adequately reflect the combinatorics of such families of partitions $P_{i}$. 
9.2. Følner functions for iterated wreath product of groups. Given groups $\Gamma_{1}$ and $\Gamma_{2}$, denote (with a minor abuse of notation) by $\Gamma_{1}^{\Gamma_{2}}$ the restricted Cartesian power group consisting of the functions $\theta: \Gamma_{2} \rightarrow \Gamma_{1}$ with finite supports (i.e., equal to id $\in \Gamma_{1}$ away from a finite subset in $\Gamma_{2}$ ) and observe that both $\Gamma_{1}$ and $\Gamma_{2}$ naturally act on $\Gamma_{1}^{\Gamma_{2}}$, where $\Gamma_{1}$ is implemented in $\Gamma_{1}^{\Gamma_{2}}$ by the functions trivial (i.e., $=\mathrm{id} \in \Gamma_{1}$ ) away from id $\in \Gamma_{2}$. The group generated by these two actions is called the wreath product of $\Gamma_{1}$ and $\Gamma_{2}$, which we denote by $\Gamma_{1}^{2 \Gamma_{2}}$. (The customary notation for the wreath product is $\Gamma_{1}<\Gamma_{2}$ and/or $\Gamma_{2}<\Gamma_{1}$ ).

There is a natural exact sequence

$$
1 \rightarrow \Gamma_{1}^{\Gamma_{2}} \rightarrow \Gamma_{1}^{2 \Gamma_{2}} \rightarrow \Gamma_{2} \rightarrow 1,
$$

where $\Gamma_{1}^{\Gamma_{2}}$ is given the coordinate-wise group structure (as the Cartesian product of $\Gamma_{2}$ copies of $\Gamma_{1}$ ) and where $\Gamma_{1}$ is embedded into $\Gamma_{1}^{\Gamma_{2}}$ and, hence, into $\Gamma_{1}^{2 \Gamma_{2}}$ by functions $\theta: \Gamma_{2} \rightarrow \Gamma_{1}$ such that $\theta\left(\gamma_{2}\right)=\mathrm{id} \in \Gamma_{1}$ for $\gamma_{2} \neq \mathrm{id} \in \Gamma_{2}$. This sequence is split by the obvious embedding $\Gamma_{2} \rightarrow \Gamma_{1}^{2 \Gamma_{2}}$.

Observe that the group $\Gamma_{1}^{2 \Gamma_{2}}$ is finitely generated if $\Gamma_{1}$ and $\Gamma_{2}$ are finitely generated, while the subgroup $\Gamma_{1}^{\Gamma_{2}} \subset \Gamma_{1}^{2 \Gamma_{2}}$ is infinitely generated for infinite groups $\Gamma_{2}$.

The wreath product is a binary operation on groups, now written $\Gamma_{1}<\Gamma_{2}$ for $\Gamma_{1}^{2 \Gamma_{2}}$, that is neither commutative nor associative: the composed wreath product of $k$ groups is determined by the way we put the brackets. Here are three (most symmetric) examples for $k=8$,

$$
\begin{aligned}
& \Gamma_{1} 2\left(\Gamma_{2} 2\left(\Gamma_{3} 2\left(\Gamma_{4} 2\left(\Gamma_{5} 2\left(\Gamma_{6} 2\left(\Gamma_{7} 2 \Gamma_{8}\right)\right)\right)\right)\right)\right), \\
& \text { (.).(.). (..) } \\
& \left.\left(\left(\left(\left(\left(\Gamma_{1}<\Gamma_{2}\right)<\Gamma_{3}\right)<\Gamma_{4}\right)<\Gamma_{5}\right)<\Gamma_{6}\right)<\Gamma_{7}\right)<\Gamma_{8} \\
& \text { (.).).)...) }
\end{aligned}
$$

and

$$
\left.\left(\left(\Gamma_{1}<\Gamma_{2}\right) \succ\left(\Gamma_{3}<\Gamma_{4}\right)\right)<\left(\left(\Gamma_{5}<\Gamma_{6}\right) \succ\left(\Gamma_{7}<\Gamma_{8}\right)\right) . \quad \quad(\ldots(0)) \ldots\right)
$$

The $E_{\circ}$-functions (obviously) follow the composition rule for partitioning slices and since the $E_{\mathrm{o}}$-function of the simple wreath product equals the numerical 2-product, i.e., $L_{1}\left\langle L_{2}:=L_{2} \cdot L_{1}^{L_{2}}\right.$, the $E_{\circ}$-functions of the iterated wreath products relative to the subgroups $\Gamma_{i}$ equal the corresponding composed 2-product with the same bracketing as for the corresponding $\Gamma$. That is,

$$
E\left(L_{1}, L_{2}, L_{3} \ldots\right)=L_{1} \prec\left(L_{2}\left(2\left(L_{3} 2 \ldots\right)\right)\right) \ldots
$$

for .(.(.)...

Now we invoke the lower bound of the Følner functions by $E_{\circ}$-functions (see 4.5) and obtain the

Erschler inequality for wreath products. The multivariable Folner function of an iterated wreath product of $\Gamma_{i}$ is bounded from below by the correspondingly composed 
2-product of $c_{i} F_{i}\left(\varepsilon_{i} n_{i}\right)$, where $F_{i}$ are the $F \phi$ lner functions of $\Gamma_{i}$ and $c_{i}, \varepsilon_{i}$ are arbitrary positive constants satisfying $\sum_{i}\left(\varepsilon_{i}+c_{i}\left(1-\varepsilon_{i}\right)\right)<1$.

Remark. The above $\left(F^{\langle F}\right)$ serves slightly better for the simple products,

$$
F_{\circ}\left(n_{1}, n_{2} ; \Gamma_{1}^{2 \Gamma_{2}}\right) \geq d_{2} F_{2}\left(\varepsilon_{2} n\right)\left(d_{1} F_{1}\left(\varepsilon_{1} n\right)\right)^{d_{2} F_{2}\left(\varepsilon_{2} n\right)},
$$

where,

$$
d_{i}=\frac{1-\varepsilon_{1}-\varepsilon_{2}}{1-\varepsilon_{i}}
$$

for arbitrary positive numbers $\varepsilon_{i}, i=1,2$ satisfying $\varepsilon_{1}+\varepsilon_{2}<1$.

Similarly, $\left(F_{\text {. }}\right)$ implies that

$$
F_{\bullet}\left(n_{1}, n_{2} ; \Gamma_{1}^{\gtrless \mathbb{Z}}\right)=n_{2} F_{\bullet}\left(n_{1}, \Gamma_{1}\right)^{n_{2}} .
$$

Question. Is there a sharp bound on the Følner functions of the (composed) wreath products more general than $\left(F_{\text {。 }}\right)$ ?

Remarks. (a) The question of evaluating the Følner functions for wreath products was raised by A. Vershik in [29]. C. Pittet and L. Saloff-Coste found (see [27]) a lower bound on the Følner function $F_{\circ}$ of the wreath products of Abelian by finite groups (i.e., $F i n^{2 A b}$ ); their inequality, albeit asymptotically non-sharp, provided the first examples of $F_{\circ}$ with super-exponential growth.

The Vershik question was answered in [9] with the inequality $\left(F^{2 F}\right)$, where the iterated case followed by induction. (The constants $c_{i}$ and $\varepsilon_{i}$ in $c_{i} F_{i}\left(\varepsilon_{i} n_{i}\right)$ in [9] are smaller than those imposed by the above $\sum_{i}\left(\varepsilon_{i}+c_{i}\left(1-\varepsilon_{i}\right)\right)<1$ and the inductive argument makes them dependent on the shape of bracketing with the best result for $(() \ldots())$ where the induction goes from $i$ to $2 i$. We see the relevance of the constants below.)

(b) The isoperimetric inequalities for iterated wreath products of finite groups $\Gamma$ and graphs (see [9]) and their modifications provide examples of graphs with expandertype isoperimetry (see [19]).

(c) Wreath Products over homogeneous spaces. The above inequalities for groups straightforwardly generalize (see [9]) to homogeneous spaces, e.g. to $X_{2}=\Gamma_{2} / \Gamma_{2}^{\prime}$ and the group $\Gamma \supset \Gamma_{1}^{X_{2}}$ generated by the restricted Cartesian power group $\Gamma_{1}^{X_{2}}$, where $\Gamma_{2}$ naturally acts on $\Gamma_{1}^{X_{2}}$ :

the max-F申lner function $F_{\circ}\left(n ; \Gamma / \Gamma_{2}^{\prime}\right)$ of the action of $\Gamma$ on $\Gamma / \Gamma_{2}^{\prime}=\Gamma_{1}^{2 X_{2}}$ as well as the max-Folner of the group $\Gamma$ itself are bounded in terms of those for $\Gamma_{1}$ and the action of $\Gamma_{2}$ on $X$ by

$$
F_{\circ}(n ; \Gamma) \geq F_{\circ}\left(n ; \Gamma / \Gamma_{2}^{\prime}\right) \geq F_{2} \cdot F_{1}^{F_{2}}
$$

for $F_{1}(n)=\frac{1}{2} F\left(\frac{n}{3} ; \Gamma_{1}\right)$ and $F_{2}(n)=\frac{1}{2} F\left(\frac{n}{3} ; X_{2}\right)$. 
(d) The lower bound by $\left(L_{1}^{2 L_{2}}\right)$ for $E_{\mathrm{o}}$-function of the wreath powers (of sets) combined with 4.4 yields a proof of the following version of $F^{2 F}$ for the Følner functions associated to $I_{\circ}^{\text {incr }}$, the maximal monotone increasing minorant of $I_{\circ}$ :

$\boldsymbol{F}^{\text {incr }}$-inequality. $F_{\circ}^{\text {incr }}\left(\Gamma_{1}^{2 \Gamma_{2}}, n\right) \geq F_{\circ}^{\text {incr }}\left(\Gamma_{2}, \frac{n}{2}\right)\left(F_{\circ}^{\text {incr }}\left(\Gamma_{1}, \frac{n}{2}\right)\right)^{F_{\mathrm{o}}^{\text {inc }}\left(\Gamma_{2}, \frac{n}{2}\right)}$.

This inequality $F^{\text {incr }}$ is slightly sharper than $F^{2 F}$ if $\Gamma_{i}$ are (necessarily infinite) groups with monotone increasing profiles $I_{\circ}(r)$. But $F^{2 F}$ meaningfully applies to finite groups where the inequality $F^{\text {incr }}$ is vacuous.

Isoperimetric inequality for groups with distorted wreath product subgroups. Take an iterated wreath product $\Gamma$ of $\Gamma_{i}$ and let $\alpha$ be an endomorphism of $\Gamma$ induced by injective endomorphisms $\alpha_{i}$ of $\Gamma_{i}$. Then $\Gamma_{i}$ can be distorted in the group $\Gamma^{\alpha}=\Gamma \times_{\alpha} \mathbb{Z}$.

For instance, if $\Gamma_{i}$ are free Abelian and $\alpha_{i}\left(\gamma_{i}\right)=2 \gamma_{i}$ for all $i$ and $\gamma_{i} \in \Gamma_{i}$, then $\Gamma_{i} \subset \Gamma^{\alpha}$ are exponentially distorted and so

$$
F_{\circ}\left(n, \Gamma^{\alpha}\right) \sim F_{\circ}(\exp (n), \Gamma) .
$$

9.3. Infinite 2 - and $\uparrow$-products. Probably the Cartesian products and the wreath products are the only 2 -variable functorial operations obtained by adding universal relations to free products of pairs of groups in the category of amenable groups, but there are further "products" in the category of ordered groups obtained as follows.

Denote by $\Gamma^{2 n}=\Gamma^{(2 n}$ the $n$-th wreath iteration .(.).(... with all $\Gamma_{i}=\Gamma_{1}$, let $I$ be an arbitrary countable ordered set and assign to each $n$-element subset $J \subset I$ the group $\Gamma_{1}^{2 J}=\Gamma_{1}^{2 n}$. This assignment is obviously functorial for inclusions of subsets and we can define the inductive limit for $J$ exhausting $I$, denoted $\Gamma_{1}^{2 I}$.

If $I$ is infinite then this group is infinitely generated; but if we take some finitely generated group $\Gamma_{2}$ of order preserving transformations transitively acting on $I$, then the group generated by $\Gamma_{1}^{2 I}$ and $\Gamma_{2}$ is finitely generated. If we take an ordered group $\Gamma_{2}$ for $I$, we denote the resulting group $\Gamma$ by $\Gamma_{1}^{\uparrow \Gamma_{2}}$ (in agreement with Knuth's arrow notation) and regard it as the "first order wreath product" (we regard $?$ as the zero order product) of the two groups. (See [9] for the case $I=\mathbb{Z}$.)

We iterate this $n$ times and, by passing from $\Gamma_{1}^{\uparrow n}=\Gamma_{1}^{\left(\uparrow_{n}\right)}$ to the inductive limit, arrive at next product $\Gamma=\Gamma_{1}^{\uparrow \uparrow \Gamma_{2}}$, where we need both groups to be ordered. Similarly we define the $\uparrow \uparrow \uparrow$-product, etc.

The above construction equally applies to the right bracketing .).).)..., but it does not (seem to) work as it stands for the ... (()()) ... -bracketing: we need a finitely generated order preserving group $\Gamma_{2}$ transitive on all $n$-tuples of points in $I$. There is a finitely generated group with this property, the Richard Thompson group, but this group is unknown to be amenable or non-amenable. (We are keen on getting large amenable group by such procedures.) On the other hand the finitely generated 
group obtained in such a way is rather amusing; for instance, it contains the finite iterated wreath products with all positions of the brackets and the same applies to $\uparrow \uparrow \ldots$-products of any given order.

Question. (a) Do the $E_{\circ}$-functions (and/or the Følner functions) of the partitions of the above $\Gamma$ into the orbits of $\Gamma_{i}$ equal (asymptotically equivalent to) the corresponding "arrowings" of the function $n_{1} 2 n_{2}=n_{2} n_{1}^{n_{2}}$ ?

Here is a (little) step in this direction.

Isoperimetric inequality for $\boldsymbol{\Gamma}=\boldsymbol{\Gamma}_{\mathbf{1}} \uparrow \boldsymbol{\Gamma}_{\mathbf{2}}$. Let $\Gamma^{k} \subset \Gamma$ be the subgroup generated by the union of $G_{1}^{\gamma_{i}} \subset \Gamma, i=1,2, \ldots, k$, for some $k$ elements in $\Gamma_{2}$ of the $G_{2}$-length $\leq l$, where $G_{i}$ are given generating subsets in $\Gamma_{i} \subset \Gamma, i=1,2$.

The max-Følner function of the group $\Gamma^{k}$, which is isomorphic to the left-iterated, i.e., $\cdots 2\left(2\left(\ldots\right.\right.$, bracketed wreath product of $\Gamma_{1}$ with itself, is bounded from below by the $k$-iterated 2-composition of $F_{k}(n)=1 / 2 k F_{\circ}\left(n / 2 k ; \Gamma_{1}, G_{1}\right)$, denoted $\imath_{k} F_{k}(n)$, where the max-boundary refers to all $k\left|G_{1}\right|$ generators in $\Gamma_{k} \subset \Gamma$.

Thus, the Følner function of $\left(\Gamma, G_{1} \cup G_{2}\right)$ is bounded from below by $F_{k}(n / 3 l)$ (compare with 6.1, p. 563).

Take $\gamma_{i}$ from $G_{2}^{l} \subset \Gamma_{2}$ such that $l \cdot\left|G_{2}^{l}\right| \sim n / 10$ for the appropriate $l=l(n)$, and regard the cardinality $\left|G_{2}^{l}\right|$ as the function $\varphi(n)$. For example, if the growth function $G_{\circ}(l)=\left|G_{2}^{l}\right|$ of $\Gamma_{2}$ equals $l^{m}$, then $\varphi(n) \sim n^{\frac{m}{m+1}}$, and if $\Gamma_{2}$ has exponential growth, then $\varphi(n) \sim n / \log (n)$.

Then, by the argument in 6.1,

the Folner function $F_{\circ}(n ; \Gamma)$ is bounded from below by $\lambda_{\varphi(n)} 2^{n}$, which is equivalent to the $\varphi(n)$-iterated exponential function $\exp (\exp (\ldots(\exp (2)) \ldots))$.

Questions. (a) Apparently, one has a poor understanding of the combinatorial structure of the orbit partitions of such groups as $\Gamma_{1} \uparrow_{i} \Gamma_{2}$, where the attractive feature of this structure is being universal, defined for arbitrary ordered sets rather than groups, and where the necessity of the order is due to the fact that the 2-product, a two-variable functor on sets, is non-commutative.

If $i=2$, there are two partitions $P_{1}$ and $P_{2}$ of $\Gamma_{1} \uparrow \Gamma_{2}$, where the combinatorics is faithfully encoded by the bipartite graph on the vertex set of the slices of $P_{i}$ with the edges corresponding to the pairs of intersecting slices.

What is the combinatorics of such graphs? For example, what are the spectra (of the combinatorial Laplacians) of such graphs constructed with finite ordered sets?

Does the graph theoretic picture reveal anything, or it is better to think in terms of partitions?

What does correspond to the concepts "measure" and/or "entropy" that would lead to something like the Shannon inequalities? 
Do the groups like $\Gamma_{1} \uparrow_{i} \Gamma_{2}$ embed (preferably functorially) into finitely presented ordered groups $\Gamma$ (similar to the Thomson group $\Gamma \supset \mathbb{Z} \imath \mathbb{Z}$ ), where one also wants $\Gamma$ to be amenable for amenable $\Gamma_{i}$ ?

Are there other "interesting" multi-variable operations (functors?) in the category of finitely generated groups that preserve the classes of amenable and/or of orderable groups? What are the functors from the category of finite ordered sets to the category of such operations on groups?

An example of a one variable operation is $\Gamma \leadsto \Gamma^{\prime} \supset \Gamma$, where $\Gamma^{\prime}$ consists of the bijective maps $\Gamma \rightarrow \Gamma$ which are equal to left translations in $\Gamma$ away from a finite subset. (If $G \subset \Gamma$ generates $\Gamma$ then $\Gamma^{\prime}$ is generated by $G$ and the permutations of $G$; probably, $\left.F_{\mathrm{o}}\left(\Gamma^{\prime}\right) \sim\left(F_{\mathrm{o}}(\Gamma)\right) !.\right)$

There are similar extensions of $\Gamma$ with "simple" bijective maps $\Gamma \rightarrow \Gamma$, e.g., the maps that are translations on a subgroup $\Gamma_{0} \subset \Gamma$ and that fix $\gamma \in \Gamma \backslash \Gamma_{0}$, but making such an extension functorially is problematic.

(b) It seems impossible to continue the $\uparrow$ hierarchy in the category of ordered groups. For example, it is unclear whether all groups $\mathbb{Z}^{\left(\uparrow_{k}\right.}$ embed into an amenable group on two generators. (In general, one does not seem to know which countable families of finitely generated amenable groups embed into a single finitely generated amenable group, while the answer is an easy "yes for all" in the class of all groups by the small cancellation theory for relatively hyperbolic groups.)

Yet, one can go several steps up if the background group $\Gamma_{2}$ admits an infinite strictly increasing family of subgroups, say $\Gamma_{3} \subset \Gamma_{4} \subset \cdots \subset \Gamma_{k} \subset \cdots$, where, similarly to the $k$-commutator relations in 8.2 , one adds the relations defining the $k$-th arrowing operation to each $\Gamma_{k}$. Then the resulting group $\Gamma$ (that can be arranged with three generators) contains all $\mathbb{Z}^{\left(\uparrow_{k}\right.}$ and, probably, its Følner function grows (at least) as fast as the Ackermann function.

It is unclear up to which ordinals one can go in a similar manner keeping groups finitely generated. (Every elementary amenable group $\Gamma$ built by normal extensions and inductive limits is characterized by the ordinal that expresses the minimal "number" of steps in such description of $\Gamma$; it seems unknown whether all countable ordinals correspond to finitely generated $\Gamma$.) Apparently, one needs a group theoretic realization of more powerful (implicit) recursion schemes starting from groups with some "self similarity", e.g., finitely generated (even better finitely presented ) amenable groups $\Gamma$ containing (better, isomorphic to) $\Gamma^{2 \Gamma}$; but such groups are yet to be found (or shown not to exist).

\section{Brunn-Minkowski inequalities}

Recall that the Euclidean Brunn-Minkowski inequality provides the following lower bound on the product (called Minkowski sum and usually denoted "+" rather than "." 
in the Abelian case) of measurable subsets $Y_{1}, \ldots, Y_{m}$ in $\mathbb{R}^{k}$,

$$
\left|Y_{1} \cdot Y_{2} \cdot \ldots \cdot Y_{m}\right|^{\frac{1}{k}} \geq\left|Y_{1}\right|^{\frac{1}{k}}+\left|Y_{2}\right|^{\frac{1}{k}}+\cdots+\left|Y_{m}\right|^{\frac{1}{k}} .
$$

(See the survey article by Gardner [12].)

Remark on nilpotent groups. The classical proof of $\left(\mathrm{BM}^{\frac{1}{k}}\right)$ extends to the nilpotent case and yields $\left(\mathrm{BM}^{\frac{1}{k}}\right)$ in the simply connected nilpotent (and, probably, solvable) case Lie groups $\Gamma$ of dimension $k$.

10.1. Asymptotic Brunn-Minkowski inequalities for discrete groups. The inequality $\left(\mathrm{BM}^{\frac{1}{k}}\right)$, unlike the isoperimetric inequality, does not directly pass to cocompact discrete subgroups $\Gamma$, as it is seen, for example, for subsets $Y_{1}$ and $Y_{2}$ in $\Gamma=\mathbb{Z}^{2}$ that are both contained in a cyclic subgroup. To correct for this one may stabilize the $Y$ 's by taking their powers

$$
Y^{N}=\underbrace{Y \cdot Y \cdot \ldots \cdot Y}_{N} \subset \Gamma .
$$

(Such stabilization in the context of polynomial rings was suggested to me by Andrei Suslin.)

If $\Gamma=\mathbb{Z}^{k}$, then the majority of points in the power set $Y^{N}$ of every finite subset $Y \subset \mathbb{Z}^{k}$ are obtained by intersecting the subgroup $\Gamma_{Y} \subset \Gamma$ generated by $Y$ with a convex subset $C_{N} \subset \mathbb{R}^{k} \supset \mathbb{Z}^{k}$, where the subsets $\frac{1}{N} C_{N}$ converge in $\mathbb{R}^{k}$ for $N \rightarrow \infty$.

Then $\left(\mathrm{BM}^{\frac{1}{k}}\right)$ in $\mathbb{R}^{k}$ implies the following asymptotic inequality in $\mathbb{Z}^{k}$ :

$$
\left(\left|Y_{1}^{N} \cdot Y_{2}^{N} \cdot \ldots \cdot Y_{m}^{N}\right|\right)^{\frac{1}{k}}-\left(\left|Y_{1}^{N}\right|^{\frac{1}{k}}+\left|Y_{2}^{N}\right|^{\frac{1}{k}}+\cdots+\left|Y_{m}^{N}\right|^{\frac{1}{k}}\right) \geq-\varepsilon_{N} N^{k}\left(\mathrm{BM}_{\varepsilon}^{\frac{1}{k}}\right)
$$

where $\varepsilon_{N} \rightarrow 0$ for $N \rightarrow \infty$.

Question. What happens for finite $N$ ? Is there a meaningful bound on $\left|\varepsilon_{N}\right|$ independent of $Y$ 's?

Are there BM-type inequalities between "monomials" $\left|Y_{1}^{N_{1}} \cdot Y_{2}^{N_{2}} \cdot \ldots \cdot Y_{m}^{N_{m}}\right|$ ?

Naively, one expects that BM-type lower bounds on $\left|Y_{i}^{N}\right|$, say $\left|Y_{i}^{2}\right| \geq 2^{k}\left|Y_{i}\right|$, may yield such a bound on $\left|Y_{1} \cdot Y_{2} \cdot \ldots \cdot Y_{m}\right|$ but it seems rather subtle already for $\mathbb{Z}$.

Limit form of $\left(\mathbf{B M}_{\varepsilon}^{\frac{1}{k}}\right)$ for $N \rightarrow \infty$. Since $\mathbb{Z}^{k}$ is commutative, so is the Minkowski sum (written here as product) and $Y_{1}^{N} \cdot Y_{2}^{N}=\left(Y_{1} \cdot Y_{2}\right)^{N}$. Using this and $\mathrm{BM}_{\varepsilon}^{\frac{1}{k}}$, one concludes that for every finite $Y \subset \mathbb{Z}^{k}$ there exists the limit

$$
|Y|_{\infty}:=\lim _{N \rightarrow \infty} \frac{1}{N^{k}}\left|Y^{N}\right|,
$$


and the inequality $\mathrm{BM}_{\varepsilon}^{\frac{1}{k}}$ takes a more compact form

$$
\left|Y_{1} \cdot Y_{2} \cdot \ldots \cdot Y_{m}\right|_{\infty}^{\frac{1}{k}} \geq\left|Y_{1}\right|_{\infty}^{\frac{1}{k}}+\left|Y_{2}\right|_{\infty}^{\frac{1}{k}}+\cdots+\left|Y_{m}\right|_{\infty}^{\frac{1}{k}} . \quad\left(\mathrm{BM}_{\infty}^{\frac{1}{k}}\right)
$$

Remark. Notice that this inequality, unlike the Euclidean BM, does not imply the isoperimetric inequality for general subsets $Y \subset \Gamma$ since the stabilization renders subsets essentially convex.

Nilpotent groups. If $\Gamma$ is a torsion-free nilpotent group with the growth function $G_{\circ}(n) \sim n^{l}$, then the above argument yields that

$$
\left(\left|Y_{1}^{N} \cdot Y_{2}^{N} \cdot \ldots \cdot Y_{m}^{N}\right|\right)^{\frac{1}{l}}-\left(\left|Y_{1}^{N}\right|^{\frac{1}{l}}+\left|Y_{2}^{N}\right|^{\frac{1}{l}}+\cdots+\left|Y_{m}^{N}\right|^{\frac{1}{l}}\right) \geq-\varepsilon_{N} N^{l}, \quad\left(\mathrm{BM}_{\varepsilon}^{\frac{1}{l}}\right)
$$

where $\varepsilon_{N} \rightarrow 0$ for $N \rightarrow \infty$.

Questions. (a) Is there some version of $\mathrm{BM}_{\infty}^{\frac{1}{k}}$ for nilpotent groups?

In general, let $\Gamma$ be any countable group, $Y_{i}, i=1,2, \ldots, m$, finite subsets in $\Gamma$ and consider all "non-commutative monomials" $\left|Y_{i_{1}}^{N_{1}} \cdot Y_{i_{2}}^{N_{2}} \cdot \ldots \cdot Y_{i_{n}}^{N_{n}}\right|$ for all $n=1,2, \ldots$ (This $|\ldots \cdot \ldots|$ for fixed $Y_{i}, i=1, \ldots, m$, makes a submultiplicative function on the free semigroup with $m$ generators.)

(b) What are $\Gamma$-universal BM-type inequalities between these monomials? (Here "universality" allows dependence of these inequalities on $\Gamma$ and $N_{j}$ but not on $Y_{i}$ except for some mild conditions on $Y_{i}$ such as being symmetric generating subsets in $\Gamma$, where such an assumption simplifies the picture already in the Abelian and nilpotent cases.)

Example. Let $\Gamma$ be a finitely generated group of exponential growth and set

$$
|Y|_{\log }=\lim _{N \rightarrow \infty} \frac{1}{N} \log \left|Y^{N}\right|
$$

(c) When does the following asymptotic equality hold true?

$$
\lim _{N \rightarrow \infty} \frac{1}{N} \log \left(\left|Y_{i_{1}}^{a_{1} N} \cdot \ldots \cdot Y_{i_{n}}^{a_{m} N}\right|\right)=a_{1}\left|Y_{i_{1}}\right|_{\log }+\cdots+a_{m}\left|Y_{i_{n}}\right|_{\log } . \quad \quad \quad \text { (=) }
$$

This is tautologically true if all $Y_{i}$ are equal and it is also obvious for pairs of subsets $Y_{1}$ and $Y_{2}$ in the free group $F_{k}$, where $Y$ equals the standard symmetric generating set (with $2 k+1$ elements) in $F_{k}$.

Equation $(=)$ also holds true for symmetric generating sets $Y_{i}$ in hyperbolic groups $\Gamma$. For example let $m=2$, regard $Y_{1}^{N}$ and $Y_{2}^{N}$ as $N$-balls with respect to the word metrics associated with the generating sets $Y_{1}$ and $Y_{2}$, and think of the product $Y_{1}^{N} \cdot Y_{2}^{N}$ as the $N$-neighborhood of the first ball in the metric defined by $Y_{2}^{N}$. 
Both balls are quasiconvex and the rate of growth of the $N$-neighborhoods of a quasiconvex set $U \subset \Gamma$ for every word metric is proportional to the rate of growth of the balls themselves, while the initial condition for the growth is given by the cardinality of the boundary of $U$,

$$
\left|U \cdot Y_{2}^{N}\right| \sim\left|\partial_{Y_{2}}(U)\right| \cdot\left|Y_{2}^{N}\right| .
$$

This is seen with the finiteness (Markov) property of the boundary types of boundaries of balls in hyperbolic groups.

In our case of $U=Y_{1}^{N}$ the cardinality of the boundary of $U$ is roughly the same as the cardinality of $U$ itself; this concludes the proof in the present special case and the general case goes along the same lines.

Remark. There is a parallel discussion for probability measures on $\Gamma$ instead of subsets, where the Minkowski products are replaced by convolutions of measures and the cardinalities of subsets by the entropies of these measures, but I have not worked out any example of a BM-inequality in this context.

10.2. Linearized BM-inequalities for biorderable groups. Given an algebra $A$ over some field $\mathbb{F}$ and linear subspaces $D_{1}, D_{2} \subset A$, we denote by $D_{1} * D_{2} \subset A$ the $\mathbb{F}$-linear span of the products $d_{1} d_{2}$ for all $d_{1} \in D_{1}$ and $d_{2} \in D_{2}$, and we reiterate the above discussion with the $D$-monomials

$$
\left|D_{i_{1}}^{N_{1}} * D_{i_{2}}^{N_{2}} * \cdots * D_{i_{n}}^{N_{n}}\right| \text { instead of }\left|Y_{i_{1}}^{N_{1}} \cdot Y_{i_{2}}^{N_{2}} \cdot \ldots \cdot Y_{i_{n}}^{N_{n}}\right|,
$$

where $|\ldots|$ in the $D$-context stands for the $\mathbb{F}$-rank of a linear space rather than cardinality as in the $Y$-situation.

We are most interested in the case of $A$ being the group algebra of some $\Gamma$ where we expect the $D$-monomials satisfy BM-type inequalities similar to those for $Y$ monomials, and we shall prove this below for biorderable groups:

a group $\Gamma$ is called biorderable if it admits an order invariant under left and right translations.

Examples. (a) Abelian groups without torsion are obviously biorderable.

(b) Central extension of biorderable groups by biorderable are clearly biorderable.

(c) Inductive and projective limits of biorderable groups are obviously biorderable.

It follows that the residually torsion-free nilpotent groups are biorderable. In particular free groups are biorderable. Also the fundamental groups of surfaces, except for the projective plane and the Klein bottle, are bi-orderable. (The fundamental group of the Klein bottle is clearly left orderable. See [25] for more geometric examples of (bi)-orderable groups.) 
Recall (see 3.1) the correspondence $f \mapsto x_{f}^{\min }$ for functions $f$ on ordered sets $X$ and observe that

$$
x_{f * g}=x_{f} \cdot x_{g}
$$

for $X$ being a biordered group $\Gamma$ and $*$ denoting the (convolution) product in the group algebra $A$ of $\mathbb{F}$-valued functions with finite support on $\Gamma$.

It follows that the assignment

$$
A \supset D \mapsto Y_{D}^{\min } \subset \Gamma
$$

(of the sets of non-zero "diagonal entries" of triangular bases to finite dimensional subspaces $D \subset A$ ) satisfies

$$
Y_{D_{1} * D_{2}}^{\min } \supset Y_{D_{1}}^{\min } \cdot Y_{D_{2}}^{\min }
$$

(This was pointed out to me by Dima Grigoriev for polynomial rings with the lexicographic order on monomials.)

Therefore,

if $\Gamma$ is a biorderable group (or semigroup) then its group algebra A over any field has the same BM-profile as $\Gamma$ : every BM-type inequality between $Y$-monomials passes to the corresponding inequality for D-monomials.

Then, by combining this with the asymptotic Brunn-Minkowski inequality $\left(\mathrm{BM}_{\infty}^{\frac{1}{k}}\right)$ for a free Abelian (or nilpotent) group $\Gamma$, we arrive at

\section{Linearized BM-inequality for the group algebra $A$ of $\Gamma$.}

$$
\left|D_{1} * D_{2} * \cdots * D_{m}\right|_{\infty}^{\frac{1}{k}} \geq\left|D_{1}\right|_{\infty}^{\frac{1}{k}}+\left|D_{2}\right|_{\infty}^{\frac{1}{k}}+\cdots+\left|D_{m}\right|_{\infty}^{\frac{1}{k}} . \quad\left(\mathrm{BM}_{\mathrm{lin}}^{\frac{1}{k}}\right)
$$

Remark. The Euclidean BM-inequality, as well as a more powerful AlexandrovFenchel inequality for mixed volumes for convex sets, can be proved by an algebrageometric (or by an essentially equivalent complex analytic) argument due to Hovanski and Teissier (see [13] and references therein), where BM is reduced to a similar inequality between the ranks of the spaces $H^{0}\left(\mathscr{L}_{i}\right)$ of regular sections of positive line bundles $\mathscr{L}_{i}, i=1,2, \ldots, m$, (over toric varieties associated to the sets of the integer points of convex polyhedra) and the tensor products of $\mathscr{L}_{i}$. Probably, $\left(\mathrm{BM}_{\mathrm{lin}}^{\frac{1}{k}}\right)$ (and also Alexandrov-Fenchel) remains valid, under suitable assumptions, for linear subspaces $D_{i} \subset H^{0}\left(\mathscr{L}_{i}\right)$, and it would be also interesting to find something similar for non-commutative nilpotent groups $\Gamma$.

10.3. BM-inequality for fast decaying functions on $\boldsymbol{\Gamma}$. Since the construction of the triangular bases needs only the one-sided bound on the support of functions on $\Gamma=\mathbb{Z}^{k}$, the above argument shows that 
the inequality $\left(\mathrm{BM}_{\mathrm{lin}}^{\frac{1}{k}}\right)$ holds in the (commutative) algebra $A_{\infty}$ of formal power series in $k$ variables over any field.

It follows that

$\left(1_{\mathrm{alg}}\right)$ the inequality $\left(\mathrm{BM}_{\mathrm{lin}}^{\frac{1}{k}}\right)$ holds true in the algebra of regular functions on an arbitrary irreducible $k$-dimensional algebraic variety,

$\left(2_{\mathbb{C}}\right)\left(\mathrm{BM}_{\text {lin }}^{\frac{1}{k}}\right)$ holds for finite dimensional linear spaces $D_{i}$ in the algebra $A$ of $\mathbb{C}$-valued function on $\mathbb{Z}^{k}$ with exponential decay at infinity (relative to the word length in $\mathbb{Z}^{k}$ ), where the product in $A$ is given by the convolution.

In fact, such functions are Fourier transforms of real analytic functions $f$ on the torus $\mathbb{T}^{k}$ the above applies to the Taylor expansions $\tau_{t}(f)$ of $f$ at some point $t \in T$.

Remarks. (a) The super-polynomial rate of decay is not sufficient for $\left(\mathrm{BM}_{\mathrm{lin}}^{\frac{1}{k}}\right)$ in $\left(2_{\mathbb{C}}\right)$, since the ring of $C^{\infty}$-function on $\mathbb{T}^{k}$ has zero divisors.

(b) One needs significantly less from an algebra $A$ of smooth functions than analyticity in order to have $\left(\mathrm{BM}_{\mathrm{lin}}^{\frac{1}{k}}\right)$. For example, if the Taylor map $\tau_{t}: A \rightarrow A_{\infty}$ for $f \mapsto \tau_{t}(f)$ is injective, i.e., if $\tau(f) \neq 0$ unless $f=0$ (at least in a neighborhood of $t \in T)$, then $\left(\mathrm{BM}_{\text {lin }}^{\frac{1}{k}}\right)$ holds in $A$.

Moreover, $\left(\mathrm{BM}_{\mathrm{lin}}^{\frac{1}{k}}\right)$ holds in algebras $A$ of quasi-analytic functions, where the germs of function at a point $t$ can be graded by ordinal numbers but I do not know the most general condition on the (relative or absolute) decay rate that would ensure the injectivity of $\tau$ (extended to infinite ordinals) and/or the inequality $\left(\mathrm{BM}_{\mathrm{lin}}^{\frac{1}{k}}\right)$.

Remark. Linear spaces $D$ of $\mathbb{C}$-valued functions on a general group $\Gamma$ with sufficiently fast (superexponential?) decay are likely to satisfy the same type of BMinequalities as spaces of functions with finite supports, but the approximation argument from 1.7 does not seem to apply in the present case.

$\left(3_{\mathbb{Q}_{p}}\right)$ The inequality $\left(\mathrm{BM}_{\mathrm{lin}}^{\frac{1}{k}}\right)$ holds true for the decaying $\mathbb{F}$-valued function $f: \Gamma \rightarrow \mathbb{F}$ for an arbitrary discrete valuation field $\mathbb{F}$, e.g., for $\mathbb{F}=\mathbb{Q}_{p}$.

Proof. Let $f(x)$ be a non-zero analytic function that is a power series,

$$
f(x)=\sum_{-\infty<i<+\infty} a_{i} x^{i} \quad \text { for } a_{i} \in \mathbb{F},
$$

with decaying coefficients $\left\|a_{i}\right\| \rightarrow 0$ for $i \rightarrow \pm \infty$, and let $m(f)$ denotes the number of the coefficients $a_{i}$ with the maximal norm, i.e., with $\left\|a_{i}\right\|=\|f\|:=\max _{i}\left\|a_{i}\right\|$.

If $f=(1-x)^{d} g(x)$ for an analytic function $g$, then $\|g(x)\| \leq\|f(x)\|$ and $d \leq m(f)-1$. 
In fact, the coefficients $b_{i}$ of $\left(\sum_{i} a_{i} x^{i}\right) /(1-x)$ are the partial sums,

$$
b_{i}=\sum_{j \leq i} a_{j}
$$

and if $b_{j} \rightarrow 0$ for $j \rightarrow+\infty$, then $m(f(x) /(1-x)) \leq m(f)-1$; thus, at most $m(f)-1$ divisions are possible in the rings of analytic functions, since $\left\|(1-x)^{d}\right\| \leq$ $\|(1-x)\|^{d}=1$ and $\left\|(1-x)^{d} g(x)\right\| \leq\|g(x)\|$.

This implies the (trivial special case of)

Division theorem. Each non-zero analytic function $f$ admits a unique product decomposition $f(x)=(1-x)^{d} g(x)$, where $g(x)$ is an analytic function with $g(1)=\sum_{i} b_{i} \neq 0$.

Next consider a central extension

$$
1 \rightarrow \mathbb{Z} \rightarrow \Gamma \rightarrow \Gamma_{1} \rightarrow 1
$$

and observe (this was explained to me by Ofer Gabor) that this division automatically extends to the algebra $\mathcal{A}(\Gamma)$ of "F-analytic functions" $f=\sum_{\gamma \in \Gamma} a(\gamma)$ with decaying coefficients $a(\gamma)$, where the role of $x \in \mathcal{A}$ is played by one of the two generators +1 or -1 in $\mathbb{Z} \subset \Gamma \subset \mathcal{A}$, say by +1 :

every non-zero $f \in \mathcal{A}(\Gamma)$ is uniquely decomposable as $f=(1-x)^{d} g$, where $g \in \mathcal{A}(\Gamma)$ is such that its image in $\mathcal{A}\left(\Gamma_{1}\right)$ under the push-forward homomorphism does not vanish.

This implies, in particular, that, by induction on $k$, the algebra $\mathcal{A}\left(\mathbb{Z}^{k}\right)$ embeds (by the counterpart of the above $\tau$-homomorphism) into the algebra of formal power series and the claim of $\left(3_{\mathbb{Q}_{p}}\right)$ follows.

Remark on non-commutative groups $\Gamma$. Let $I \subset A$ denote the augmentation ideal (generated by $1-g_{j}$ for some generators $g_{j} \in \Gamma$ ) of the group algebra $A$ of $\Gamma$ and let $A_{i}$ denote the quotients $A / I^{i}$ for the powers $I^{i}$ of $I$.

Then $\rightarrow A_{i} \rightarrow A_{i-1} \rightarrow \cdots \rightarrow A_{1}=\mathbb{F}$ forms a projective system of nilpotent algebras that are finite dimensional for finitely generated $\Gamma$, where the projective limit of this system is denoted by $A_{\infty}$ and is equipped with the usual topology. If $\Gamma=\mathbb{Z}^{k}$, then $A_{\infty}$ equals the space of formal power series (in $k$ commuting variables) at the point $t=\mathbf{1}=(1,1, \ldots, 1)$ in the torus $\mathbb{T}^{k}$.

More generally, if the intersection of $I^{i}$ equals zero (e.g., if $\Gamma$ is a torsion-free nilpotent or a free group), then $A_{\infty}$ is regarded as the formal completion of $A$. The above division argument shows that the algebra $\mathcal{A}(\Gamma)$ of $\mathbb{F}$-analytic functions embeds to $A_{\infty}$ and a similar conclusion holds for Archimedean fields (e.g., $\mathbb{R}$ and $\mathbb{C}$ ) for "analytic" functions with a suitably defined radius of convergence $>1$. Thus the isoperimetric problems in $\mathcal{A}(\Gamma)$ reduce to those in $A_{\infty}$. 
Question. When does the algebra $A_{\infty}$ satisfy the same isoperimetric and BM inequalities as $A$ itself? Is it true in the case where the intersection of the powers of the ideal $I$ equal zero? (This "nil-intersection" condition is quite strong: if the underlying field $\mathbb{F}$ has characteristic zero it is close to the group being residually nilpotent, and for a finite field it looks even more restrictive.)

\section{References}

[1] F. Almgren, Optimal isoperimetric inequalities. Indiana Univ. Math. J. 35 (1986), 451-547. Zbl 0585.49030 MR 0855173

[2] L. Bartholdi, On amenability of group algebras, I. Israel J. Math. 168 (2008), 153-165.

[3] A. Belov and R. Mikhailov, Free algebras of lie algebras close to nilpotent. Preprint 2008. arXiv:0805.0723v2

[4] T. Coulhon and L. Saloff-Coste, Isopérimétrie pour les groupes et les variétés. Rev. Mat. Iberoamericana 9 (1993), 293-314. Zbl 0782.53066 MR 1232845

[5] E. G. Effros, New perspectives and some celebrated quantum inequalities. Preprint 2008. arXiv:0802.0006v2

[6] G. Elek, The amenability of affine algebras. J. Algebra 264 (2003), 469-478. Zbl 1022.43001 MR 1981416

[7] G. Elek, On the analytic zero divisor conjecture of Linnell. Bull. London Math. Soc. 35 (2003), 236-238. Zbl 1027.20002 MR 1952401

[8] G. Elek, The amenability and non-amenability of skew fields. Proc. Amer. Math. Soc. 134 (2006), 637-644. Zbl 02237897 MR 2180879

[9] A. Erschler, On isoperimetric profiles of finitely generated groups. Geom. Dedicata 100 (2003), 157-171. Zbl 1049.20024 MR 2011120

[10] A. Erschler, Piecewise automatic groups. Duke Math. J. 134 (2006), 591-613. Zbl 05077494 MR 2254627

[11] E. Friedgut, Hypergraphs, entropy and inequalities. Amer. Math. Monthly 111 (2004), 749-760. MR 2104047

[12] R. J. Gardner, The Brunn-Minkowski inequality. Bull. Amer. Math. Soc. (N.S.) 39 (2002), 355-405. Zbl 1019.26008 MR 1898210

[13] M. Gromov, Convex sets and Kähler manifolds. In Advances in differential geometry and topology, World Scientific, Singapore 1990, 1-38. Zbl 0770.53042 MR 1095529

[14] M. Gromov, Topological invariants of dynamical systems and spaces of holomorphic maps: I. Math. Phys. Anal. Geom. 2 (1999), 323-415. Zbl 01428101 MR 1742309

[15] M. Gromov, Random walk in random groups. Geom. Funct. Anal. 13 (2003), 73-146. Zbl 1122.20021 MR 1978492

[16] M. Gromov, Singularities, expanders, hyperbolic geometry and fiberwise homology of maps. In preparation. 
[17] M. Gromov, Mendelian dynamics and Sturtevant's paradigm. In Geometric and probabilistic structures in dynamics, Contemp. Math. Ser., Amer. Math. Soc., Providence. RI, to appear.

[18] M. Gromov and V. Milman, Generalization of the spherical isoperimetric inequality to uniformly convex Banach spaces. Compositio Math. 62 (1987), 263-282. Zbl 0623.46007 MR 0901393

[19] S. Hoory, N. Linial, and A. Wigderson, Expander graphs and their applications. Bull. Amer. Math. Soc. (N.S.) 43 (2006), 439-561. Zbl 05302790 MR 2247919

[20] O. E. Lanford III, Entropy and equilibrium states in classical statistical mechanics. In Statistical mechanics and mathematical problems (Battelle Seattle 1971 Rencontres), Lecture Notes in Phys. 20, Springer-Verlag, Berlin 1973, 1-113.

[21] P. W. Michor, D. Petz, and A. Andai, On the curvature of a certain Riemannian space of matrices. Infin. Dimens. Anal. Quantum Probab. Relat. Top. 3 (2000), 199-212. Zbl 1031.53109 MR 1812696

[22] D. Witte Morris, Amenable groups that act on the line. Algebr. Geom. Topol. 6 (2006), 2509-2518. Zbl 05118602 MR 2286034

[23] M. Ohya and D. Petz, Quantum entropy and its use. Corr. 2nd printing, Texts and Monographs in Physics, Springer-Verlag, Berlin 2004. Zbl 0891.94008 MR 1230389

[24] D. Petz and C. Sudár, Extending the Fisher metric to density matrices. In Geometry of present days science (Aarhus, Denmark, 1997), World Scientific, Singapore 1999, 21-33. Zbl 0939.62008

[25] D. Rolfsen, Ordered groups and topology. Lecture Notes, Luminy, June 2001. http://www.math.ubc.ca/ rolfsen/papers/luminynotes/lum.pdf

[26] M. B. Ruskai, Another short and elementary proof of strong subadditivity of quantum entropy. Rep. Math. Phys. 60 (2007), 1-12. Zbl 05240230 MR 2355462

[27] C. Pittet, and L. Saloff-Coste, Amenable groups, isoperimetric profiles and random walks. In Geometric group theory down under (Canberra, 1996), Walter de Gruyter, Berlin 1999, 293-316. Zbl 0934.43001 MR 1714851

[28] N. Th. Varopoulos, L. Saloff-Coste, and T. Coulhon, Analysis and geometry on groups. Cambridge Tracts in Math. 100, Cambridge University Press, Cambridge 1992. Zbl 0813.22003 MR 1218884

[29] A. Vershik, Amenability and approximation of infinite groups. Selecta Math. Soviet. 2 (1982), 311-330. Zbl 0533.22007 MR 0721030

Received May 20, 2007; revised June 11, 2008

M. Gromov, Institut des Hautes Études Scientifiques, Le Bois-Marie 35, route de Chartres, 91440 Bures-sur-Yvette, France

E-mail: gromov@ihes.fr 